\title{
RELIABLE BROADCASTING IN VEHICULAR NETWORKS
}

\author{
Mozhdeh Gholibeigi
}




\section{Reliable Broadcasting in Vehicular Networks}

Mozhdeh Gholibeigi 
Graduation committee:

Chairman:

Promoter:

Promoter:

Members:

Prof. dr. A. Vinel

Prof. dr. R.D. van der Mei

Prof. dr. ir. S.M. Heemstra de Groot

Dr. ir. M. de Graaf

Prof. dr. ir. B.R.H.M. Haverkort
Prof. dr. J.N. Kok

Prof. dr. ir. G.J. Heijenk

Prof. dr. J.L. van den Berg

Halmstad University

Vrije Universiteit Amsterdam

Eindhoven University of Technology

University of Twente

University of Twente

Funding sources:

EU FP7 Mobility 2.0 - 314129

DSI Ph.D. Thesis Series No. 18-021

DIGITAL SOCIETY Institute on Digital Society

INSTITUTE $\quad$ P.O. Box 217, $7500 \mathrm{AE}$

Enschede, The Netherlands

ISBN 978-90-365-4690-4

ISSN 2589-7721 (DSI Ph.D. thesis Series No. 18-021)

DOI $10.3990 / 1.9789036546904$

https://doi.org/10.3990/1.9789036546904 


\title{
Reliable Broadcasting in Vehicular Networks
}

\author{
DISSERTATION \\ to obtain \\ the degree of doctor at the University of Twente, \\ on the authority of the rector magnificus, \\ Prof. dr. T.T.M. Palstra, \\ on account of the decision of the graduation committee, \\ to be publicly defended \\ on Thursday the $13^{\text {th }}$ December 2018 at 10:45 hours
}

by

\section{Mozhdeh Gholibeigi}

Born on the $18^{\text {th }}$ March 1982

in Isfahan, Iran 
This dissertation has been approved by:

Prof. dr. ir. G.J. Heijenk (Supervisor)

Prof. dr. J.L. van den Berg (Supervisor) 


\section{Acknowledgments}

I feel blessed to be surrounded by people who have been supporting me during all stages of my life. I would like to express my gratitude to those who in particular have been supporting me by various means during my $\mathrm{Ph}$.D. studies over the last couple of years.

I would like to start by giving a big thank you to Prof. Yevgeni Koucheryavy, the supervisor of my M.Sc. thesis, who sparked up this journey. Such a nice and memorable visit we had in Milan that headed to starting my Ph.D. in Design and Analysis of Communication Systems (DACS) research group at the University of Twente, with his recommendation.

I express my sincere appreciations to my noble promoters Prof. Geert Heijenk and Prof. Hans van den Berg who this thesis would not have been possible without all their support, not only scientifically but equally important by their courteous attitude all the time.

Prof. Boudewijn Haverkort, the eminent chair of our group, with his kind manner and persistent support of all members.

Dr. Dmitri Moltchanov, the supervisor of my M.Sc. thesis, who I have been collaborating with during my Ph.D. studies as well.

My committee members, Prof. Alexey Vinel, Prof. Rob van der Mei, Prof. Sonia Heemstra de Groot, Prof. Maurits de Graaf and Prof. Boudewijn Haverkort for accepting to be in my committee and their valuable comments on my thesis.

I have been lucky to pursue my Ph.D. in a group with very kind and competent colleagues who I have shared nice times and fruitful discussions with. In particular, my office mates Mitra, Bernd and Sarwar. Our lovely Jeanette with all her support and care like a mother. She was always making things happen fast and flawless.

My colleagues from the Mobility 2.0 project who I learned a lot and enjoyed fun times during our project trips.

My dear paranymphs Jair Cardoso de Santanna and Justyna Chromik for their support and help during the past month.

My friends from home country who we collected nice memories together during these years while far away from the families; Mitra and Siavash, with whom we are like a family. Zhaleh and Saeed, Elahe and Farhad, who I have 
shared a lot with. Our trips could not be forgotten. Elham, Leila, Neda and Mohammad, Hajar and Meysam, Sina, Mahroo, Alireza and Barbara, Mina and Amir, Maral and Aidin, Mohammadreza and Anna, Sadaf and Mojtaba, Niloofar, Davood, Hassan, Zahra and Alireza, Maryam and Hamed.

My parents, brothers and sisters in-law with their kind heart and good vibes sent over miles.

My parents, my true treasure who they could not have been any better. With all their affection and support, making me feel strong and capable during all stages of my life. They always gave me the freedom and all I needed to follow my dreams and make them happen. Simply, I cannot thank them enough for all they did for me. My only sister beloved Mozhgan, with a heart of gold, cheering me up all the time. She has been always supportive and caring with precious advices. My brother in-law Hamed, who have always been supportive and encouraging during these years. My sweet nephews Anis and Tanin, the bright stars bringing so much positive energy and joy into my life.

And my love Morteza who is my best friend. We have been passing through a long journey, with strength and tenderness. He has always been there for me. He is reassuring and makes me feel so strong by his persistent support and provident mind. 


\begin{abstract}
Vehicular transportation is an integral part of today's life. In this respect, Intelligent Transport Systems (ITS) applications relying on vehicular communications provide means for increased efficiency and safety of vehicular transportation. ITS utilize advanced information and communication technologies in order to serve many novel application types, targeting improvement of traffic situations on the roads. By increasing the level of automation and assisting human drivers, they contribute to higher traffic safety and reduced congestion and environmental impact. Wireless communication among vehicles, the socalled vehicular networking, is the main enabler for ITS applications. Vehicular broadcasting refers to dissemination of data from a single node to all other nodes within the scope of a vehicular network and is a common communication type that many ITS applications rely on.
\end{abstract}

Assuring reliable delivery of broadcast data is of paramount importance for many and in particular safety-critical ITS applications, built upon this type of communication. That is, delivery of broadcast data within reasonable time to all intended nodes of a vehicular network. Direct Short Range Communication (DSRC) based on the Institute of Electrical and Electronics Engineers (IEEE) 802.11p standard is considered as the main wireless communication technology to enable vehicular communication, including broadcasting. However, IEEE 802.11p-based broadcast is defined as a best-effort service, lacking an acknowledgment technique. Targeting reliability improvement of vehicular broadcasting may lead to inefficiency. For instance, redundant retransmissions or acknowledgments overload the limited wireless medium with excess traffic. Hence, to be considered in this regard, is the cost at which broadcast reliability is achieved. Motivated by this, in this thesis, we focus on the reliability requirement of vehicular broadcasting. After providing a background to the scope of this thesis, we first propose an End-to-End (E2E) reliability assurance mechanism based on a sequence checking module where receivers can detect missing packets and explicitly request them. Such an approach provides broadcast reliability, while at the same time not overloading the network with redundant traffic. Using absorbing Markov chains, we analytically model the functionality of the proposed mechanism and analyze its performance in the context of a single-hop vehicular communication scenario based on the IEEE $802.11 \mathrm{p}$ standard. We 
further validate our analytical analysis via simulations. At the next step, we extend our earlier work by considering application of the proposed E2E reliability assurance mechanism in the context of a multi-hop vehicular communication scenario based on the IEEE 802.11p standard. For this, we first analytically model multi-hop data dissemination throughout the network by means of developing a closed form recursive function, quantifying the probability of network nodes having obtained broadcast data. Accordingly, we assess the error recovery performance of the E2E reliability assurance mechanism, applied upon multihop broadcasting, by means of analytical analysis, based on Markov chains and Bayesian networks. Using simulations, we further validate our analytical modeling. The results of our analysis show that the proposed reliability assurance mechanism performs efficiently, in both single-hop and multi-hop scenarios, by imposing little burden of error recovery even for high number of receivers.

Besides lacking a built-in acknowledgment technique, the IEEE 802.11p standard has other shortcomings, namely in terms of scalability under rather high network loads, with respect to fully suiting performance requirements of ITS applications. This has been the driver for the next stage of our work in this thesis. The 3rd Generation Partnership Project (3GPP) cellular communication system is a promising alternative for the IEEE $802.11 \mathrm{p}$ standard with all its potential to support vehicular communication, including large-scale deployment and infrastructure-based resource management capabilities. Specifically, Device-to-Device (D2D) communication technology has been introduced and further evolved in the recent releases of the $4^{\text {th }}$ generation of 3 GPP mobile networking system (i.e., Long Term Evolution (LTE)) towards supporting highperformance vehicular communications and accordingly ITS applications. D2D refers to direct communication between users in close vicinity, by utilizing the cellular radio spectrum and without traversing the infrastructure, as opposed to conventional cellular communications. Accordingly, it can result in proximity gain, resource reuse gain and hop gain. Ultra Reliable Low Latency Communication (URLLC) is one of the three main services of the next generation (i.e., 5G) mobile networking system, targeting ITS use cases which can serve D2D-based broadcast. In our work, we focus on the resource allocation aspect of the D2D communication technology and its utilization for vehicular broadcasting. Radio resource reuse, aiming efficient utilization of the scarce spectrum, is an important aspect of D2D resource allocation and in our work we propose a reuse-based resource allocation approach being adaptive to the network load and topology. That is, by taking into account the number of users seeking for D2D broadcast and their geographical distribution in the network, resources are allocated in the most efficient manner, aiming spectrum efficiency and collision avoidance, due to reuse. We model our proposed resource allocation approach and extensively evaluate its performance in comparison with a baseline resource allocation 
approach, in the context of single- and multi-cell scenarios. The results verify spectrum efficiency and reliability of the proposed resource allocation approach, in comparison with the baseline approach.

Our analytical models provide means for analysis of vehicular broadcasting and our proposed approaches of improving its reliability. This allows for efficient study of impact of various factors on the performance of reliable broadcasting. The results of this thesis provide insight into the behavior of broadcasting in vehicular networks and further solutions towards its performance and efficiency. Such results cannot only be used in the design of reliable vehicular communication mechanisms and accordingly high-performance ITS applications, but also can serve as a basis for future research in this direction. 



\section{Samenvatting}

Wegtransport is een belangrijk onderdeel van het alledaags leven. ITS kunnen gebruik maken van geavanceerde communicatie technologie om nieuwe toepassingen mogelijk te maken die de verkeerssituatie op de weg verbeteren. Door vervoer grotendeels te automatiseren is het mogelijk de veiligheid te vergroten, het aantal files te reduceren en milieuvervuiling te verminderen. Draadloze communicatie tussen voertuigen, vehicular networking genaamd, ondersteunt het grootste deel van de ITS-toepassingen. Het zenden van data van een enkel voertuig naar alle andere voertuigen in een netwerk wordt vehicular broadcast genoemd. De meeste ITS-toepassingen gebruiken deze communicatie methode.

Het is erg belangrijk voor ITS-applicaties dat de communicatie tussen voertuigen betrouwbaar is, d.w.z. broadcast data moet gegarandeerd afgeleverd worden bij andere voertuigen. De communicatie tussen voertuigen, waaronder broadcast, verloopt grotendeels, gebaseerd op de IEEE 802.11p standaard. De IEEE 802.11 p standaard garandeert echter niet dat broadcast verkeer ook daadwerkelijk aankomt bij de ontvangers omdat deze ontvangst niet bevestigen. De betrouwbaarheid van het systeem verbeteren kan negatieve effecten op de efficiëntie hebben. Berichten bevestigen en andere extra communicatie kunnen bijvoorbeeld de beperkte ruimte in de ether gereserveerd voor ITS-toepassingen overbelasten. Er moet dus rekening gehouden worden met de consequenties die het betrouwbaar maken van de communicatie hebben. In deze thesis richten we ons op de betrouwbaarheid van vehicular broadcasting. Na het geven van achtergrondinformatie stellen we een betrouwbaarheidsgarantie mechanisme voor dat gebaseerd is op een sequence checking module die ontvangers in staat stelt om gemiste data te detecteren en de zender te vragen deze opnieuw te verzenden. Dankzij deze aanpak kan data betrouwbaar verstuurd worden zonder dat het draadloze medium overbelast raakt door extra communicatie. We bouwen een analytisch model van het voorgestelde mechanisme met een Markovketen met een absorberende toestand. Met dit model analyseren we de prestaties van een single-hop ITS-scenario gebaseerd op de IEEE 802.11p standaard. Vervolgens valideren we deze analytische aanpak door middel van simulatie. Als volgende stap breiden we ons voorgaande werk uit door ons betrouwbaarheidsgarantie mechanisme te testen in een multi-hop scenario. We beginnen met 
een analytisch model van multi-hop data verspreiding in een netwerk door het opstellen van een gesloten recursieve formule. Deze formule berekent de kans dat data ontvangen is door een voertuig. Ook evalueren we de prestaties van ons betrouwbaarheidsgarantie mechanisme door middel van een analytische aanpak met behulp van Markovketens en Bayesiaanse netwerken. We valideren ons analytisch model door middel van simulaties. De resultaten van onze analyse laten zien dat ons betrouwbaarheidsgarantie mechanisme efficiënt is en het draadloze medium zelfs met een groot aantal ontvangers niet overbelast.

De IEEE 802.11p standaard heeft naast het gebrek aan betrouwbaarheid nog andere gebreken. Voor ITS-toepassingen zijn dit i.h.b. de schaalbaarheidsproblemen die optreden bij het uitwisselen van grote hoeveelheden data. Dit gebrek is het orderwerp van het volgende deel van deze thesis. Een veelbelovend alternatief voor de IEEE 802.11 p standaard is het cellulaire systeem voor mobiele communicatie dat gestandaardiseerd wordt binnen 3GPP. Voordelen van dit systeem zijn onder andere de mogelijkheid het beperkte spectrum efficiënt te verdelen. Naast de gebruikelijke communicatie tussen apparaat en zendmast ondersteunt dit systeem, sinds de 4e generatie, genaamd LTE, ook directe communicatie tussen apparaten (D2D). Deze techniek is belangrijk voor ITSapplicaties omdat appraten die zich dicht bij elkaar bevinden, in tegenstelling tot de meer bekende mobiele communicatie via zendmasten, kunnen communiceren zonder vaste infrastructuur te gebruiken. Een van de voordelen van dit systeem is dat het beschikbare spectrum vaker hergebruikt kan worden. De volgende generatie mobiele netwerken $(5 \mathrm{G})$ biedt diensten die zich specifiek richten op ITS-toepassingen, gebruik makend van URLLC. We richten ons specifiek op het toewijzen van het beschikbare spectrum voor D2D communicatie. Een van de belangrijkste middelen voor D2D is het efficiënt gebruiken van het beperkte beschikbare spectrum. We stellen een systeem voor dat zich aanpast aan de hoeveelheid verkeer in het netwerk en de huidige topologie. Door het aantal gebruikers dat data wil versturen en hun positie te gebruiken kunnen we het beschikbare spectrum van het systeem op de meest efficiënte manier toewijzen. We vergelijken een model van onze toewijzingsaanpak met een standaard aanpak. We doen dit voor scenario's met een enkele en meerdere basisstations. De resultaten laten zien dat ons voorstel het beschikbare spectrum beter verdeelt en efficiënter gebruikt dan de standaard aanpak.

Onze analytische modellen maken het mogelijk om vehicular broadcasting met onze voorgestelde aanpassingen te evalueren. Met deze modellen is het mogelijk om de invloed van verschillende factoren op de prestaties van vehicular broadcasting te evalueren. Deze thesis geeft inzicht in het gedrag van broadcasting in vehicular networks en geeft oplossingen voor problemen op het gebied van efficiëntie. De resultaten van ons werk kunnen niet alleen gebruikt worden 
voor het verbeteren van ITS-toepassingen maar ook dienen als startpunt voor verder onderzoek. 



\section{Contents}

1 Introduction 1

1.1 Intelligent transportation and vehicular networking . . . . . . . 2

1.2 Thesis objective and research questions . . . . . . . . . 3

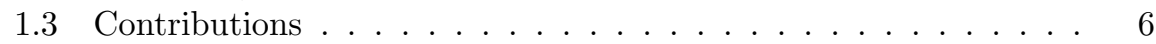

1.4 Thesis structure . . . . . . . . . . . . . 7

2 Vehicular networking $\quad 11$

2.1 ITS: a real-world demand . . . . . . . . . . . . . . . . . 13

2.2 Vehicular networking technology . . . . . . . . . . . . . 13

2.2.1 Communication domains and application demands . . . . 14

2.2.2 Short range wireless communication technology . . . . . . 15

2.2.3 5G-based D2D wireless communication technology . . . . 18

2.3 Message types . . . . . . . . . . . . . . . . . 20

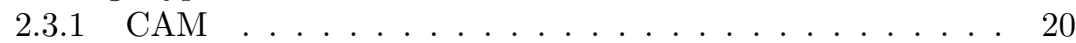

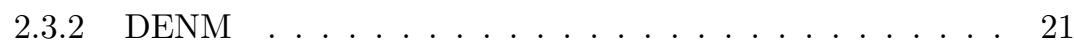

2.4 Data forwarding in vehicular networks . . . . . . . . . . . 21

2.4.1 Geographical Unicast . . . . . . . . . . . . . . . 21

2.4 .2 Geographical Anycast . . . . . . . . . . . . . . 22

2.4 .3 Broadcast . . . . . . . . . . . . . . . 22

2.5 Performance requirements and design challenges . . . . . . . . . 23

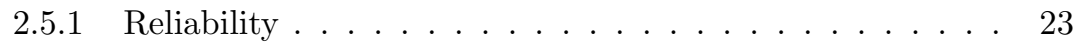

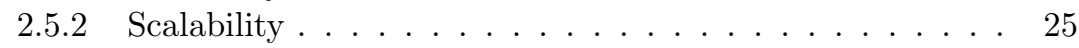

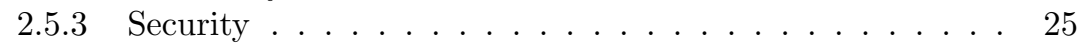

2.6 Evaluation tools . . . . . . . . . . . . . . . . 26

2.6 .1 Simulations . . . . . . . . . . . . . . . . . 27

2.6.2 Analytical modeling . . . . . . . . . . . . . . 28

3 Reliable V2V broadcast: single-hop communication scenario 31

3.1 Introduction . . . . . . . . . . . . . . . . 33

3.2 Related work . . . . . . . . . . . . . . . . . 34

3.3 End-to-End reliability mechanism . . . . . . . . . . . . . . . 36

3.4 Modeling approach . . . . . . . . . . . . . . . . . . 40

3.4.1 Considered scenario . . . . . . . . . . . . . . 40 
3.4.2 Modeling and parameterization . . . . . . . . . . . . 42

3.5 Performance analysis . . . . . . . . . . . . . . . . . . . . . . . . . . . . . 48

3.5.1 Evaluation metrics . . . . . . . . . . . . . 48

3.5 .2 Numerical results . . . . . . . . . . . . . . . . 53

3.6 Concluding remarks . . . . . . . . . . . . . . . 60

4 Reliable V2V broadcast: multi-hop communication scenario 63

4.1 Introduction . . . . . . . . . . . . . . . . . 65

4.2 Multi-hop broadcast . . . . . . . . . . . . . . 66

4.2.1 Related work . . . . . . . . . . . . . . 66

4.2 .2 Modeling approach . . . . . . . . . . . . . . 68

4.2.2.1 Considered scenario . . . . . . . . . . . . 69

4.2.2.2 Modelling and parameterization . . . . . . . 69

4.2.3 Performance analysis . . . . . . . . . . . . . . . . . 74

4.2.3.1 Preliminaries . . . . . . . . . . . . 74

4.2.3.2 Numerical results . . . . . . . . . . . . 75

4.3 Error recovery . . . . . . . . . . . . . . . . . 82

4.3.1 Related work . . . . . . . . . . . . . . 82

4.3.2 E2E reliability mechanism . . . . . . . . . . . . 83

4.3.3 Modeling approach . . . . . . . . . . . . . . . . 84

4.3.4 Performance analysis . . . . . . . . . . . . . . . 92

4.3.4.1 Evaluation metrics . . . . . . . . . . . . 92

4.3.4.2 Numerical results . . . . . . . . . . . . . 93

4.4 Concluding remarks . . . . . . . . . . . . . . . . . . . 98

5 Resource allocation for cellular D2D vehicular broadcast 100

5.1 Introduction . . . . . . . . . . . . . . . . . . . . 102

5.2 Related work . . . . . . . . . . . . . . . . 106

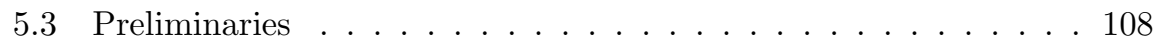

5.3.1 Resource allocation . . . . . . . . . . . . . . . 110

5.3.2 Propagation model . . . . . . . . . . . . . . 112

5.4 Adaptive resource allocation mechanism . . . . . . . . . . . . 112

5.5 Modeling approach $1 \ldots \ldots$. . . . . . . . . . . . 118

5.5.1 Performance analysis . . . . . . . . . . . . . . 120

5.5.1.1 Evaluation metrics . . . . . . . . . . 120

5.5.1.2 Numerical results .............. . 121

5.6 Modeling approach $2 \ldots \ldots \ldots$. . . . . . . . . . . . . . . . . . . . . . . . . . 125

5.6.1 Performance analysis . . . . . . . . . . . . . . 127

5.6.1.1 Evaluation metrics . . . . . . . . . . . 128

5.6.1.2 Numerical results . . . . . . . . . . . . 131

5.7 Concluding remarks . . . . . . . . . . . . . 136 
6 Conclusions 138

6.1 Contributions and Conclusions . . . . . . . . . . . . . 139

6.2 Future research directions . . . . . . . . . . . . . . . . 141

$\begin{array}{lr}\text { A Open Data Management } & 144\end{array}$

$\begin{array}{lr}\text { Bibliography } & 146\end{array}$

$\begin{array}{lr}\text { Acronyms } & 160\end{array}$

$\begin{array}{ll}\text { List of Publications } & 165\end{array}$ 



\section{Introduction}

This chapter introduces the background information and motivation of this Ph.D. thesis. It details the research objective and accordingly formulates research questions, continued with relevant approaches and main contributions highlighted. The chapter concludes with an overview of the structure of this thesis.

Evolving with a fast pace towards globalization, we are witnessing implications of the general trend the connected world in many aspects of our lives (e.g., Internet of Things (IoT), smart cities and many more). This demands data communication among various end-points which is the realization of the concept of networking. Networked end-points can share information and resources. In this respect, wireless type of networking is of significant importance, facilitating deployment, very large-scale communication and supporting new application types. Beneficiary from this progress, the automotive industry has been through significant changes and concepts such as vehicular networking and intelligent transportation has been recently defined by the community. Vehicular networking refers to wireless communication between vehicles and also road side infrastructure contributing to user comfort, road safety and traffic efficiency, via novel applications. Vehicular networking is the main enabler of ITS that provide such applications, using information and communication technologies.

Information dissemination, as the basis for vehicular networking, could be of different types where many applications rely on geographical broadcast, targeting "all" vehicles in a given area as recipients. Reliability of information dissemination is of critical importance for many application types, especially safety-critical ones. It is the objective of this thesis to study dissemination reliability of broadcast data in vehicular networks and investigate mechanisms for its improvement.

In this chapter, we introduce the topic and structure of the thesis, with the following outline. Section 1.1 presents a brief introduction to vehicular networking, ITS and data forwarding in vehicular networks. The objective of this thesis and the corresponding research questions are introduced in Section 1.2 , followed by our research contributions and thesis structure in Sections 1.3 and 1.4 , respectively. 


\subsection{Intelligent transportation and vehicular net- working}

Transportation as an integral part of today's world, facilitates our lives in many ways, while at the same time may give rise to inefficiencies and life-threatening situations (e.g., accidents and road hazards). With a rapid increase in the world's population and vehicles, one can imagine how such events can get of larger impact and fatalities. The impact on safety alone makes ITS worth considering, as according to the World Health Organization (WHO), roughly 1.25 million people died in 2015 due to traffic accidents, with an associated governmental cost of about $3 \%$ of Gross domestic product (GDP) [17]. Many governments and organizations world-wide set forth increasing transportation safety and efficiency by means of utilizing modern ITS systems that move vehicle and environment awareness to a new higher level than only human capabilities.

In general, ITS can be defined as a system that uses sophisticated communication technologies to enable communication and information exchange between vehicles and also road infrastructure, mainly as a means to improve vehicle, road and traffic safety and efficiency. One could think of the wide range of novel applications from safety to infotainment, put into practice because of such systems. Just to name a few, we can point to traffic alert dissemination, cooperative autonomous driving, dynamic route planning, obstacle warning, lane detection, collision notification, context-aware advertisement, file sharing and Internet access. In this respect, information exchange between various entities (mostly vehicles), referred to as vehicular communication, or vehicular networking, is the driving force for functioning of such systems.

Motivated by all the potential benefits of such networks for both individuals and environment, vehicular networking is an active area of research and development with multidisciplinary support from governments, industry, academia and standardization organizations.

A vehicular network consists of vehicles and possibly also fixed infrastructure nodes. Data forwarding between these nodes is the basis for functionality of such a network. Figure 1.1 shows the reference architecture of a vehicular network, with all possible communication domains [12]. Vehicles either communicate directly with no infrastructure node involved (i.e., ad hoc mode) or via infrastructure nodes.

The scope of this thesis is broadcasting in vehicular networks and evaluating its performance. Data broadcast refers to simultaneous transmission of the same data to all network nodes. It is one of the main communication types in vehicular networks and receives significant attention in the research community. There are various alternative wireless technologies to realize vehicular broadcast. DSRC, which is based on the IEEE 802.11p standard, has been 
the subject of extensive standardization and is considered as the main communication technology in vehicular networks. Cellular communication is another relevant alternative. $3 \mathrm{GPP}$ cellular systems are originally designed for mobile broadband communication with specific requirements, not necessarily the same as the requirements for ITS applications. However, given the mobile networking system as the most extensively deployed wireless technology so far and all its potential capabilities to support vehicular communication, research activities have been initiated in recent $3 \mathrm{GPP}$ standardization framework (i.e., 4G, 5G) towards introducing features supporting requirements for vehicular communication. In this thesis, we study both IEEE 802.11p-based and 5G-based vehicular broadcasting.

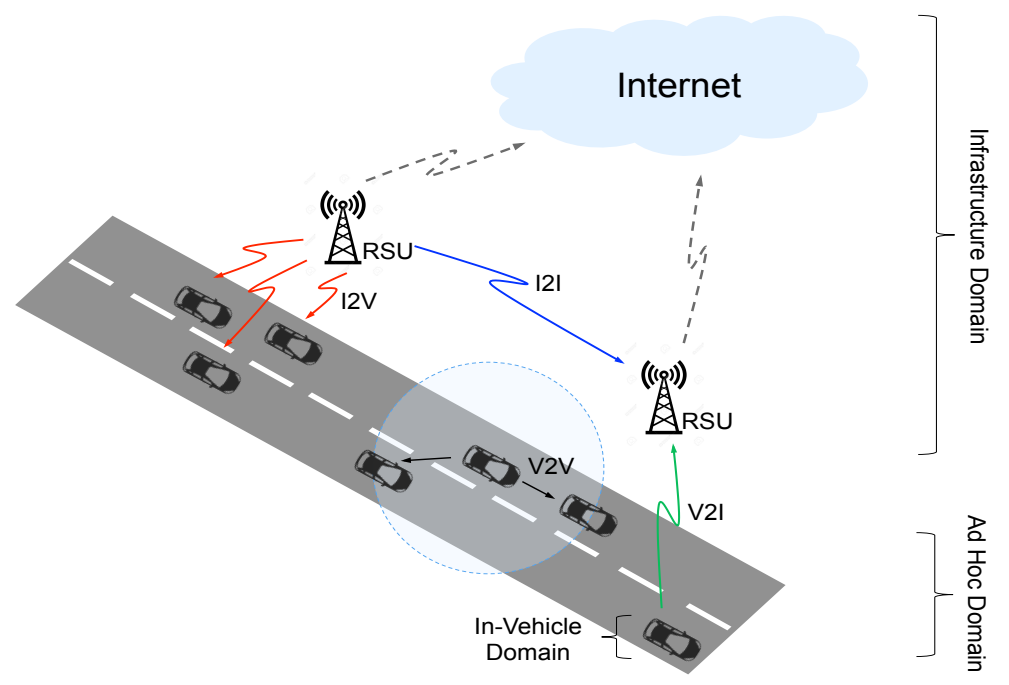

Figure 1.1: The C2C-CC reference architecture [12].

\subsection{Thesis objective and research questions}

Ensuring reliability of data dissemination is of crucial importance for many ITS applications, in particular for the ones demanding a safety action upon reception of such data. By reliability we mean integral data delivery to the intended receivers within reasonable time.

As an extension to the IEEE 802.11 standard, originally designed for indoor communication between computers in an office environment, the IEEE 
802.11p standard adds enhancements required to support ITS applications [15]. However, unlike for one-to-one (i.e., unicast) mode of communication, broadcast is based on best-effort and no acknowledgement technique is specified for that [34] [96]. This leads to challenges in providing reliable communication and renders reliable data delivery as one of the main performance concerns in Vehicle-to-Vehicle (V2V) broadcasting. Motivated by this, we put our focus on performance of vehicular broadcasting and seek solutions for increasing its reliability.

Evaluation methodologies are essential tools to gain insight on performance of a system. There are various methods for evaluating performance of a realworld vehicular broadcasting system; however, It should be noted that a realworld vehicular broadcasting system is too complex to be considered in its entirety for performance evaluation. Hence, regardless of the applied means (e.g., simulations, analytical modeling), we need to come up with a representation of the real system, where the practical details are abstracted, while keep in view the main influencing system properties. Given this fact, Figure 1.2 shows the conceptual flow of our research in this thesis, with the aim of evaluating and improving performance of vehicular broadcasting (the lower part of Figure 1.2). We achieve this via following the abstraction process presented at the upper part of Figure 1.2. That is, we first build a model of the system at high abstract level. This promotes our understanding of the real system and identify interrelations between its various components. Next, we carry out a model-driven analysis via defining performance indicators and the obtained results are later translated in terms of the system performance. 


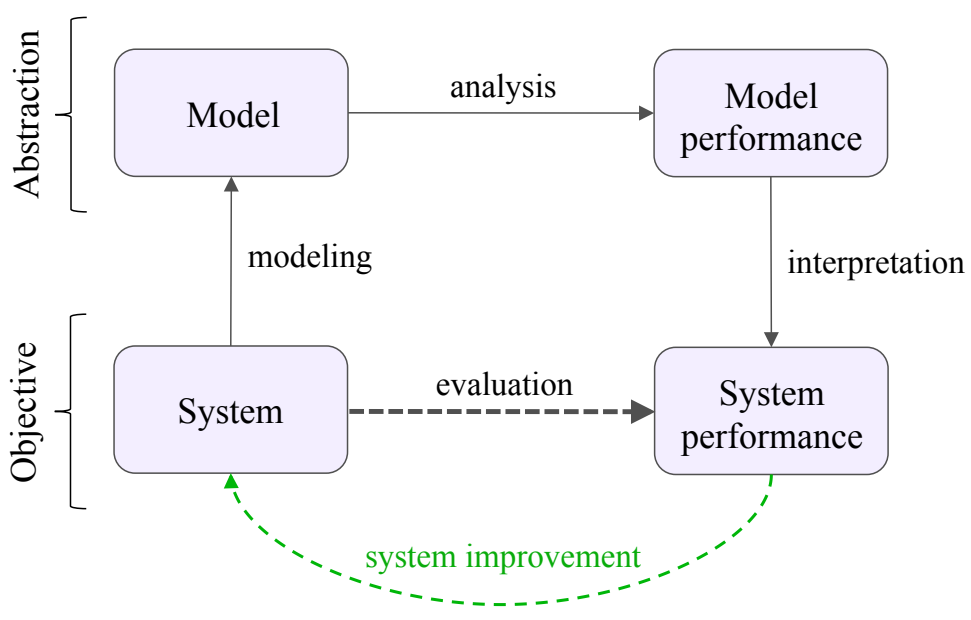

Figure 1.2: The conceptual flow of our research.

Accordingly, we formulate the main goal of this thesis as follows:

Research Goal: to model and analyze reliability of broadcasting in vehicular networks, and identify and validate solutions for its improvement.

This goal can be divided into the following three sub-goals as:

- to model the behavior of a vehicular broadcasting system.

- to analyze the system and its performance, using the model.

- to develop mechanisms for reliability improvement of vehicular broadcasting.

The defined goals entail the research questions of this thesis to be answered, as follows.

\section{Research Questions}

Modeling real-world systems is a demanding task given all the complexity of the environment they operate in and the interplay between various components. At the same time, it is a substantial task modeling of such systems providing 
insight into the system performance. This can be used to provide feedback to the system design and optimization. Accordingly, such a model on the one hand must be able to abstract practical details of the corresponding system and on the other hand capture the main properties of its functionality. This brings us to the following research question:

$\boldsymbol{R Q}$ 1: How can a system for vehicular broadcasting be modeled, with a reasonable level of abstraction?

The ultimate objective of the model is evaluating the performance of the modeled system. For this, we need to think of performance indicators to be defined, as formulated in our second research question:

$\boldsymbol{R Q}$ 2: How and in terms of which metrics can performance of the system be evaluated using the model?

The next rationale step by having the system modeled/evaluated, is seeking approaches for improving its performance. This is the focus of our third research question:

$\boldsymbol{R Q}$ 3: How can performance of vehicular broadcast be improved?

\subsection{Contributions}

The focus of this work is on performance of vehicular broadcasting, mainly in terms of reliability and ways to improve it. In this regard, the approaches proposed for reliability improvement of vehicular broadcasting can be divided into two categories, given the point in time in which they operate as:

- in-broadcast: where the action for reliability improvement is carried out in the course of broadcast, as a means to avoid loss of data.

- post-broadcast: where the action for reliability improvement is carried out upon broadcast of data, as a means of recovery of lost data.

In this work, we study both methods by proposing solutions falling in each category. Hence, inline with the objective and research questions of this thesis, the contributions of this work can be summarized as follows: 
- Propose a V2V broadcast reliability assurance approach of type postbroadcast and provide an analytical model of it which accurately captures its behavior in a single-hop V2V communication scenario, using the IEEE 802.11p standard. This approach is receiver-based. That is, the error recovery action is initiated solely on demand by the receivers in need. As a result, unlike many other approaches based on default recovery techniques, this approach keeps overhead at a reasonably low level, while significantly increasing message delivery probability. The analytical model provides means for extensive analysis and numerical results which is further validated by simulations.

- Characterize the behavior of multi-hop V2V broadcast via an analytical model of data dissemination in the network over multiple hops.

- Evaluate the aforementioned reliability assurance mechanism in a multihop communication setting, relying on the outcome of multi-hop V2V broadcasting as the starting point for error recovery. For this, we develop an analytical model which is able to reasonably parameterize the system behavior. Beside extensive numerical results, we validate the model via simulations.

- Propose a V2V broadcast reliability assurance approach of type inbroadcast which is based on cellular 5G-based D2D communication. The focus of our approach is on utilizing the radio resource management capability of cellular infrastructure during broadcast via D2D communication technology, as an effective means to avoid later collisions and accordingly lead to better broadcast performance. This is achieved through designing a resource allocation mechanism which interactively adapts to the varying network conditions, unlike most of existing approaches which rely on some fixed resource management policies. By developing a model of the proposed approach, we provide insightful results for multiple scenarios.

\subsection{Thesis structure}

The contributions of this thesis are split over the chapters shown in the schematic outline of the thesis in Figure 1.3. In the following, we provide a brief summary of chapters and refer to the corresponding publications that the chapters are based on. 


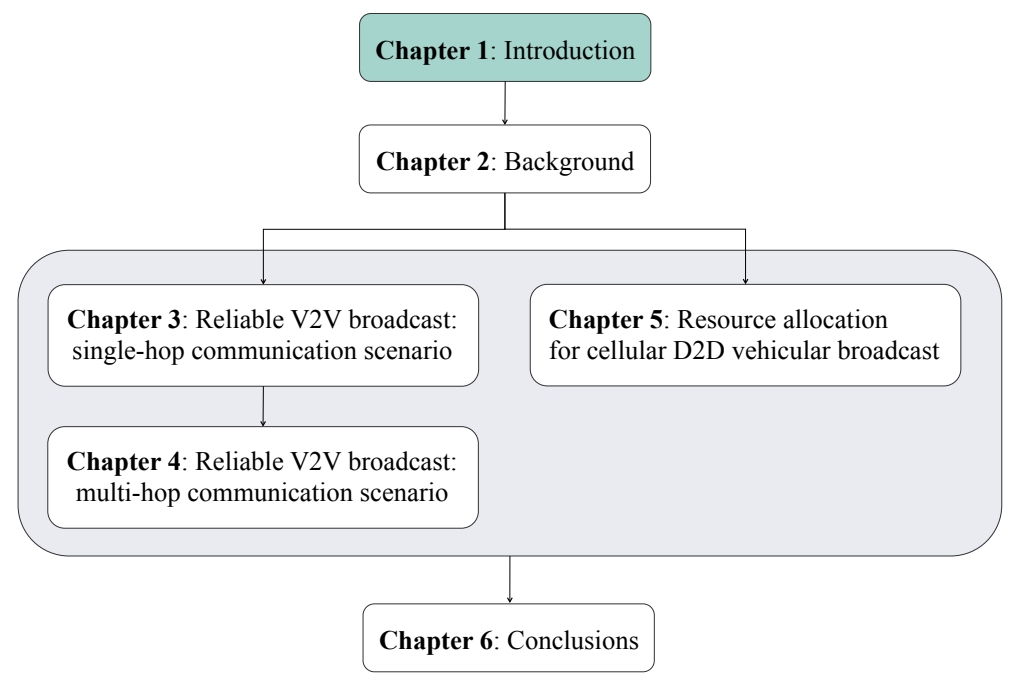

Figure 1.3: Thesis outline.

\section{Chapter 2: Vehicular networking}

This chapter presents background information on ITS systems and applications enabled by them. Further, we introduce the essential concepts of vehicular networking as the technology behind such systems. We detail the architecture, protocol stack and communication domains and technologies of these networks. Inline with the research direction of this thesis, we continue by focusing on data forwarding schemes. The chapter is wrapped up by discussing the main performance requirements of vehicular networks and challenges towards fulfilling them.

\section{Chapter 3: Reliable vehicular broadcast: a single-hop scenario}

In this chapter, we introduce a mechanism of reliability assurance for V2V broadcast data. This mechanism which is of type post-broadcast, aims at error recovery while imposing little overhead that is of particular importance for broadcast traffic. The mechanism is receiver-oriented, relying on retransmission request and reply of lost data between communicating parties. Next, we provide an analytical model of it for a single-hop communication setting. The model which is based on the absorbing Markovian chain modeling abstracts reasonably the real-world system while capturing its fundamental properties. As a result, it 
provides means for extensive analysis and numerical results and is further validated by NS3 simulations.

This chapter is based on the following peer-reviewed publications:

- M. Gholibeigi, G. Heijenk, D. Moltchanov, and Y. Koucheryavy. Analysis of a receiver-based reliable broadcast approach for vehicular networks. In IEEE Vehicular Networking Conference (VNC), 2014 [65].

- M. Gholibeigi, G. Heijenk, D. Moltchanov, and Y. Koucheryavy. Analysis of a receiver-based reliable broadcast approach for vehicular networks. Ad Hoc Networks, 37, Part 1:63 - 75, 2016. Special Issue on Advances in Vehicular Networks [66].

\section{Chapter 4: Reliable vehicular broadcast: a multi-hop scenario}

As the next step towards reliable V2V broadcasting, in this chapter we consider extending the destination area beyond the single-hop neighborhood of the source of broadcast; Hence, we focus on evaluating performance of the proposed reliability assurance mechanism in a multi-hop communication scenario. Essential for this, at first we come up with an analytical model of multi-hop broadcasting. Through a recursive function, the model provides us a quick insight into the status of individual network nodes regarding possessing broadcast data, upon data propagation in the network over multiple hops (i.e., via relay nodes). As a result of this, we arrive at a scenario where some nodes may have been failed to receive broadcast data and hence are in need of error recovery. This is the point where we develop an analytical model of the reliability assurance mechanism, as a means of error recovery for unsuccessful receivers. We benefit from fundamental characteristics of absorbing Markov modeling and Bayesian networks to build a model which is able to parameterize the main features of the corresponding system. The model allows extensive numerical computation and is validated by NS3 simulations.

This chapter is based on the following peer-reviewed publications:

- M. Gholibeigi and G. Heijenk. Analysis of multi-hop broadcast in vehicular ad hoc networks: A reliability perspective. In 2016 Wireless Days (WD), pages 1-8, March 2016 [64].

- M. Gholibeigi, M. Baratchi, H. van den Berg, and G. Heijenk. Towards reliable multi-hop broadcast in VANETs: An analytical approach. In 2016 IEEE Vehicular Networking Conference (VNC), pages 1-8, Dec 2016 [63]. 


\section{Chapter 5: Resource allocation for cellular D2D vehicular broadcast}

Motivated by limitations of existing communication technologies in providing highly reliable vehicular communication, this chapter studies the application of cellular 5G-based D2D communication as a promising alternative to the IEEE 802.11 p standard for vehicular broadcasting. In the concept of D2D communication the radio spectrum available for cellular users is shared with other users communicating directly with no cellular infrastructure involved (i.e., the socalled D2D communication). As a result, the aspect of spectrum management and sharing among users is a key factor in D2D communication performance. Accordingly, in this chapter we propose an adaptive approach of cellular resource management for improving performance of D2D vehicular broadcasting where the resource allocation policy adjusts to the varying network load and topology. Further, we provide a respective model in order to investigate performance gain obtained by the proposed approach.

This chapter is based on the following peer-reviewed publication:

- M. Gholibeigi, N. Sarrionandia, M. Karimzadeh, M. Baratchi, H. van den Berg, and G. Heijenk. Reliable vehicular broadcast using 5G device-todevice communication. In IEEE/IFIP Wireless and Mobile Networking Conference $(W M N C), 2017[67]$.

\section{Chapter 6: Conclusions}

Based on the research in earlier chapters, the thesis is concluded in the final chapter. This chapter also provides guidelines for future research directions. 


\section{Vehicular networking}

This chapter presents in more detail the concepts of ITS and vehicular networking, which we briefly introduced in Chapter 1. It serves as a general background to the topic of this thesis.

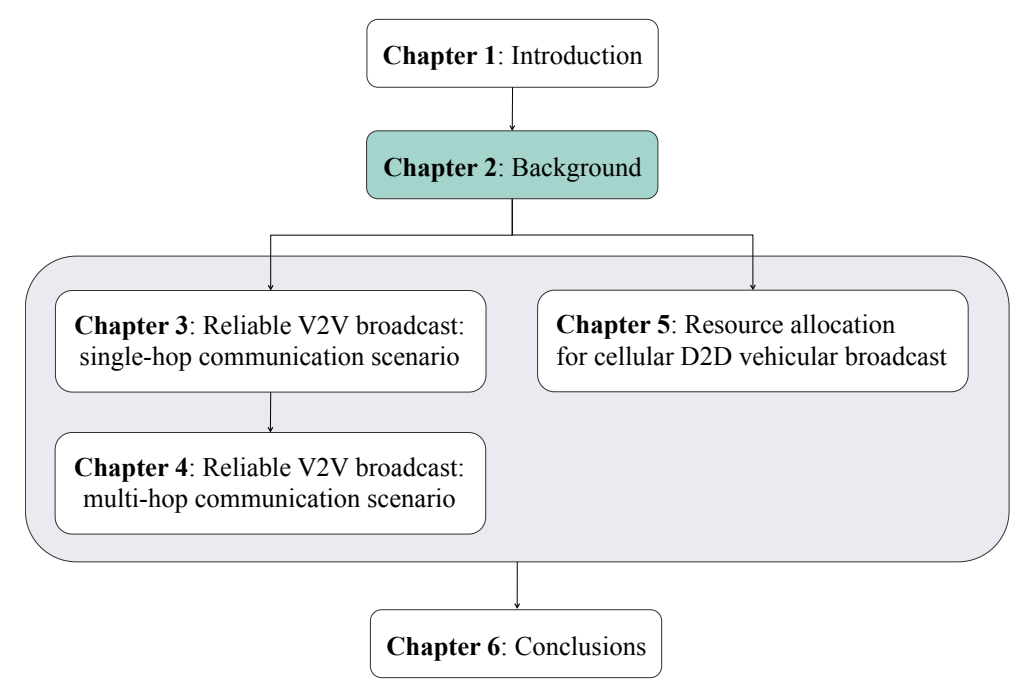


The chapter follows this outline:

- Section 2.1 presents the main concept of ITS with an emphasize on its necessity and societal impact via enabling novel applications.

- Section 2.2 discusses the underlying technologies for vehicular networking, including its protocols and communication domains and techniques.

- Section 2.4 provides information regarding data forwarding types in vehicular networks.

- Section 2.5 details performance requirements of ITS applications and accordingly vehicular communication essential to realize the promise of ITS applications regarding traffic efficiency, road safety and user comfort.

- Section 2.6 gives a brief introduction to modeling tools and methodologies for vehicular networks. 


\subsection{ITS: a real-world demand}

Parallel with the growth in world's population, the surge of technology advancements in various fields brought along higher levels of (expected) life standards and quality, but also leading to incapacity of the existing infrastructure. In the transport sector, one can identify how rapid development of urbanisation puts pressure on transportation infrastructure. Consequently, we are witnessing its immediate negative impacts such as accidents and traffic lock-down and congestion. However, it does not end with this and in long term there would be bigger problems such as environment pollution and compensation costs incurred by society and governments.

There are already initiatives regarding transformation in existing systems such as modern road construction, underground transportation and more recently electric vehicles towards addressing the mentioned issues and facilitating people and goods transfer. But that is not all and the main transformation starts at the point where ICT starts to be integrated with the physical infrastructure and intelligent systems take the place of traditional ones.

ITS refers to systems that using information, communication, data processing and sensor technologies in and between vehicles, road infrastructure and individuals' equipment (e.g., smart phones), realize a wide range of novel applications. Such applications aim to increase efficiency, safety, security, reliability and eco-friendliness of the transport network. ITS includes a wide and growing range of technologies and applications. Route scheduling, traffic management, Internet access, regular and event-based information provisioning systems (e.g., location notification, warning systems due to accidents, congestion, obstacles, road maintenance and weather conditions), electronic toll collection and advanced public transportation are just a few example applications culiminating in coordinated, fully automated driving.

ITS systems rely on the concept of vehicular networking as a means to information communication, and it is the reliability of such communication that has a major impact on the performance of these systems. In the next section, we continue by highlighting the basics of vehicular networking.

\section{$2.2 \quad$ Vehicular networking technology}

A vehicular network is a wireless network with vehicles as the main nodes communicating with each other and also with stationary infrastructure nodes. Infrastructure nodes may provide a large number of functions. For instance, they may act as relays to facilitate communication between vehicles over a longer range than direct $\mathrm{V} 2 \mathrm{~V}$ and may also provide access to the Internet and some application back offices (e.g., traffic monitoring and management centers). Road 
Side Unit (RSU) and cellular base stations (e.g., evolved NodeB (eNodeB)) are examples of infrastructure nodes. Wireless communication between nodes may be realized via various technologies. In general two main categories can be identified as cellular-based and short-range radio communication technologies. Global System for Mobile Communications (GSM), Universal Mobile Telecommunication system (UMTS), Worldwide Interoperability for Microwave Access (WiMAX) and LTE are examples of cellular communication technologies. A variety of short to medium range radio technologies have been used for vehicular communication such as Wireless Fidelity (WiFi) and IEEE 802.11p. Depending on the application types and communication domains, different technologies might be considered as appropriate. This will be discussed in detail in Section 2.2.1.

\subsubsection{Communication domains and application demands}

As presented in Section 1.1, there are three different communication domains in the reference architecture of a vehicular networking system. These include in-vehicle, ad hoc V2V and Vehicle-to-Infrastructure (V2I) domains [12], each serving specific application categories.

The first communication domain refers to information exchange between various components within a vehicle such as On Board Unit (OBU), sensors and application units. These could be of type wired (via data buses) and wireless communication. Wireless communication can be realized via an access point or in an ad hoc manner. In the first case, all devices must be connected to the access point and communicate with other components solely via the access point. Digital Enhanced Cordless Telecommunications (DECT) or IEEE 802.11 can be utilized as the communication technology. In ad hoc mode, communication can be realized via infrared light (e.g., IrDA) or radio technology (e.g., Bluetooth). Use cases for in-vehicle communication might be engine control, Anti-lock Braking System (ABS) and infotainment video streaming systems.

The ad hoc domain refers to direct communication between vehicles (via OBUs), in case of physical proximity. Otherwise, RSUs can be used to extend the coverage. Communication in this domain is fully distributed with no central coordination. The dominating communication technology in the ad hoc mode is IEEE 802.11 p based short range communication, enabling quick data exchange without the need for establishing a Basic Service Set (BSS). As an amendment to the IEEE 802.11 Wireless Local Area Network (WLAN) standard, the IEEE 802.11p standard adds enhancements towards operability for vehicular communication. However, it has drawbacks, mainly due to its inherent characteristics [84]. This will be extensively discussed in Chapter 5 of this thesis. Other wireless communication technologies, such as cellular communication are being 
evaluated as potential alternatives. In particular, it has been considered as one of the main research and development fields of the next generation of the mobile networking system (i.e., 5G), study the potential for high performance V2V communications. Platooning, collision warning and distributed traffic information systems are just few examples of the broad range of applications relying on $\mathrm{V} 2 \mathrm{~V}$ communications.

An RSU as a static node can provide vehicles with access to an infrastructure network and ultimately to the Internet. This falls into the category of V2I communication. Vehicles can get access to the infrastructure network by other means as well (e.g., mobile phones and vehicles with embedded systems can provide connectivity to cellular base stations). In this communication domain, the infrastructure may play a coordinative role by gathering information regarding traffic and road conditions and accordingly suggesting or imposing certain behaviors on particular vehicles. For instance, velocities / accelerations of vehicles and headway distances could be suggested by the infrastructure, given traffic conditions with the aim of optimizing CO2 emissions, fuel consumption and avoiding shockwave jams via smoothening traffic flows. These suggestions could be either broadcast to drivers on road displays or sent directly to vehicles via wireless connections. In a more forward looking way, one could think of these suggestions to be translated into semi-automatic actions by vehicles' control units.

Various communication technologies can be used to access the backbone networks, including the IEEE 802.11p standard, cellular, Radio Frequency IDentification (RFID) and WiFi. Internet access, map updates, reduced speed / work zone warning, red light violation warning and spot weather related information are examples of V2I applications.

\subsubsection{Short range wireless communication technology}

There are organizations around the world pursuing research and standardization activities in order to address the challenges in vehicular networking and provide means to introduce interoperable systems. Affected by geography and applied regulations, three main standardization tracks have been emerged in the USA, Europe and Japan, resulting in definitions of standard networking protocol suites. In the USA, the Wireless Access for Vehicular Environment (WAVE) protocol stack defines the IEEE 1609 family of standard protocols. The European family of standards is called European Telecommunications Standards Institute (ETSI) ITS which is adopted from the international organization for standardization's (ISO) Communication Access for Land Mobiles (CALM) architecture. As demonstrated in Figure 2.1, similar to the Open System Interconnection (OSI) reference model, there are various layers, defined with specific 
functionalities. In Japan, research activities has led to development of the Association of Radio Industries and Businesses (ARIB) and ISO CALM standards. For more detail, we refer to [123] [76] [134] [133].

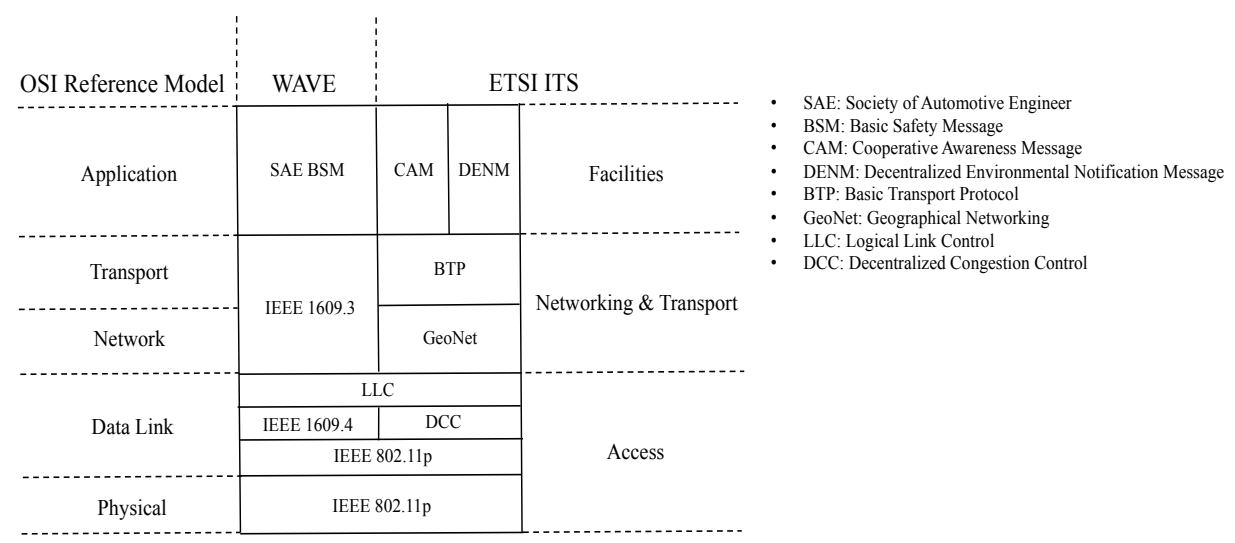

Figure 2.1: Networking protocol stacks.

\section{IEEE 802.11p}

IEEE 802.11p [73] is the main communication standard for the Physical and Data Link layer in vehicular networking. It is an extension from the IEEE 802.11 [73] standard which is designed for indoor WLAN communications. The IEEE 802.11 p standard targets modifications, enabling WAVE and accordingly supporting ITS applications. This includes direct data exchange between vehicles without the need to establish a BSS, implying no need for authentication or association. The rationale behind is the short-living communication links between vehicles, where establishing a BSS would be too time taking. One may note that one consequence of this is that the authentication and data confidentiality mechanisms provided by the IEEE 802.11 standard cannot be applied to IEEE 802.11p. Hence, they must be addressed by higher layers. Another modification is the addition of a new time management frame, allowing vehicles to be synchronized. Further, the channel allocation in the Physical layer has been modified to be more robust against the dynamics of a vehicular network such as Doppler shift.

In the US, IEEE 802.11p is the basis for DSRC [135]. DSRC is the two-way short range wireless communication technology based on the CALM architecture of the ISO standard for vehicular communication networks. It enables high data transmission which is crucial for vehicular safety applications. $75 \mathrm{MHz}$ of 
spectrum in the $5.9 \mathrm{GHz}$ frequency band has been allocated for DSRC in the US as shown in Figure 2.3. $5 \mathrm{MHz}$ out of the $75 \mathrm{MHz}$ spectrum is reserved as the guard band and 7 channels, each of $10 \mathrm{MHz}$, are defined as 1 Control CHannel $(\mathrm{CCH})$ and 6 Service CHannels $(\mathrm{SCH}) \mathrm{s}$. The $\mathrm{CCH}$ is considered for transmission of high-priority safety messages or control data, while SCHs are considered for transmission of other less demanding data. The pair of channels (174 and 176 - 180 and 182) can be combined into a single channel of $20 \mathrm{MHz}$, as channel 175 and 181, respectively.

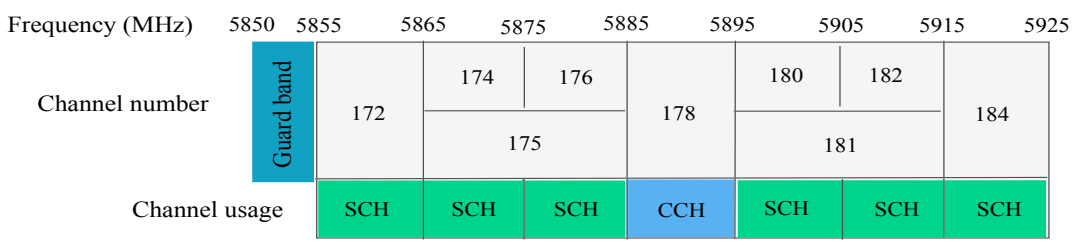

Figure 2.2: The US DSRC frequency band allocation [135].

Similarly in Europe, IEEE 802.11p is considered for the ITS-G5 standard. It enables the so-called GeoNetworking [50] in vehicular communications which is standardised by ETSI ITS. This would be further discussed in Section 2.4. Figure 2.3 shows the channel allocation for using IEEE 802.11p in Europe.

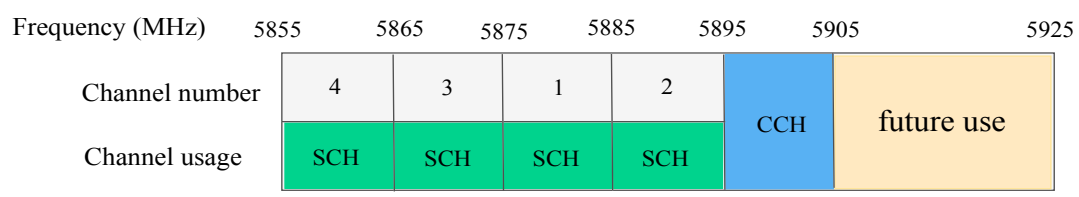

Figure 2.3: The European DSRC frequency band allocation [48].

\section{CSMA/CA}

Medium access in the IEEE 802.11p standard is adopted from the IEEE 801.11e standard which is a contention based channel access method, called Enhanced Distributed Channel Access (EDCA). EDCA defines the Carrier Sense Multiple Access / Collision Avoidance (CSMA/CA) mechanism. That is, nodes sense the medium and transmit when the channel is sensed to be idle. This significantly minimizes the possibility of collisions and makes more efficient use of the medium.

Because of the high mobility of vehicles, the Request-To-Send / Clear-ToSend (RTS/CTS) handshaking mechanism may introduce performance degra- 
dation. Hence, it has been eliminated in IEEE 802.11p. This results in the so-called hidden node problem in vehicular networks as shown in Figure 2.4.

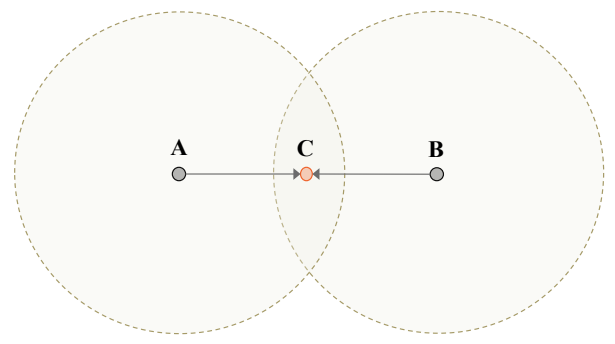

Figure 2.4: Two transmitters $\mathrm{A}$ and $\mathrm{B}$ which are out of each others coverage range, send at the same time to the receiver $\mathrm{C}$; This results in a collision at the receiver $\mathrm{C}$.

\subsubsection{G-based D2D wireless communication technology}

$\mathrm{D} 2 \mathrm{D}$ communication is a wireless communication technology of the next generation $3 \mathrm{GPP}$ mobile networking system (i.e., $5 \mathrm{G}$ ), referring to direct communication between users in proximity of each other, without uplink and downlink traverse through cellular infrastructure. Such a mechanism promises high performance in terms of high bit rates, low delays and low power consumption, due to the proximity of users. Also the reuse gain is achieved by shared utilization of radio resources cellular as well as D2D users. The hop gain, referring to the direct link communication rather than using two up and down links in the cellular mode.

D2D communications scenarios can be classified based on the utilized cellular spectrum being dedicated or shared (with cellular users) and also involvement of cellular network infrastructure such as a cellular base station (i.e., eNodeB) or a core network (i.e., Evolved Packet Core (EPC) network) in communication establishment. Figure $2.5 \mathrm{a}$ and $2.5 \mathrm{~b}$ show respectively direct and infrastructurecontrolled D2D communication alternatives. Whereas the first option can operate without cellular network coverage, infrastructure-controlled D2D communication relies on scheduling capability of cellular infrastructure for link establishment.

D2D communication has recently been the subject of study as a promising alternative to the IEEE 802.11p standard for vehicular communications [84]. Worth mentioning is in particular the central medium access management by cellular infrastructure as an efficient means for interference management as opposed to the distributed contention-based CSMA/CA in IEEE 802.11p, resulting in scalability issues. In this regard, broadcast type of D2D communication 


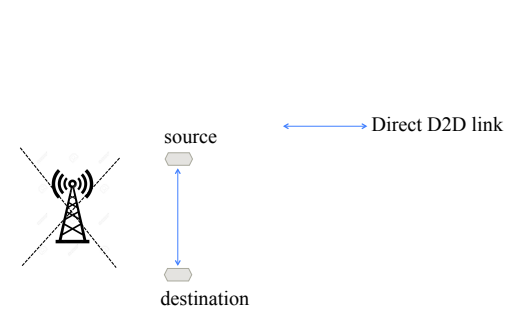

(a) Direct.

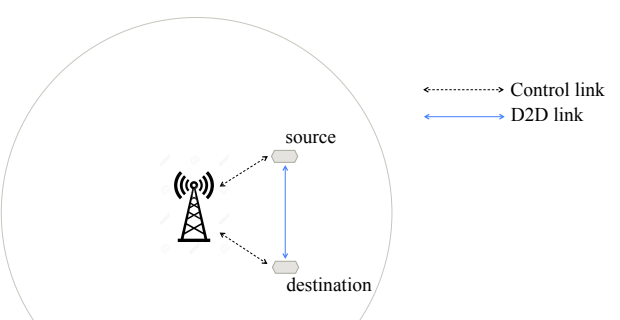

(b) Infrastracture-controlled.

Figure 2.5: D2D communication scenarios.

has been specified for serving broadcast-based ITS applications with ultra reliable and low latency communication requirements. Further detail regarding advantages of 5G-based D2D communication, compared to IEEE 802.11p-based communication, will be discussed in Chapter 5 of this thesis. Also we refer to [60] [130] [84] for information regarding design challenges and technical aspects of D2D communication.

Given D2D communication supposed to use the uplink cellular spectrum (in case of Frequency Division Duplex (FDD)) or uplink sub-frames (in case of Time Division Duplex (TDD)) [22], its physical data channel has the structure of the Physical Uplink Shared CHannel (PUSCH) (See Figure 2.6), based on Single Carrier-Frequency Division Multiple Access (SC-FDMA) scheme [22] [20]. SC-FDMA is an attractive alternative to Orthogonal Frequency Division Multiple Access (OFDMA), particularly for the uplink communications where the mobile terminal benefits from a lower peak-to-average power ratio in terms of the transmit power efficiency and lower cost amplifiers. 


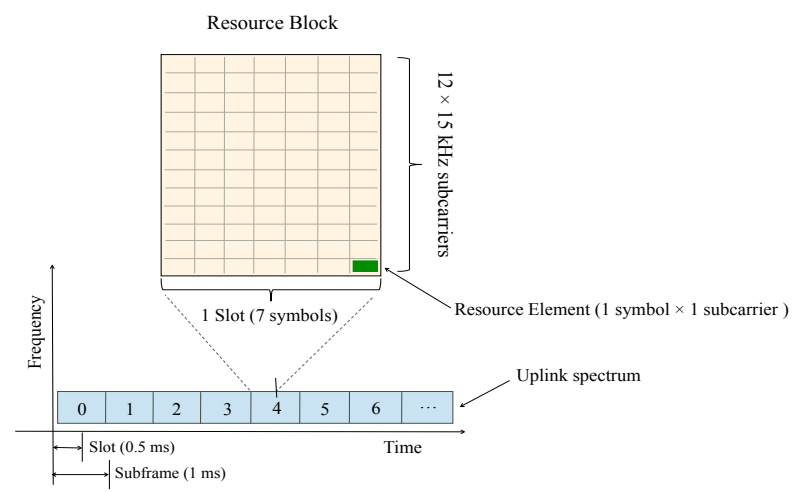

Figure 2.6: Uplink spectrum PUSCH physical channel structure (for FDD).

\section{$2.3 \quad$ Message types}

ETSI ITS specifies standards to support the development and implementation of ITS Services in order to achieve global interoperability between all ITS systems. For this, an important necessity is clear definition of data formats that is also crucial for optimal usage of the bandwidth. Various message types with different formats have been specified for different purposes by ETSI ITS standard and in what follows we briefly highlight specifications for two main messages.

\subsubsection{CAM}

Cooperative Awareness Message (CAM) [53] is defined as a basic awareness service of the Facilities layer to support cooperative ITS applications demanding regular status information about surrounding vehicles or RSUs. This is achieved by means of periodic exchange of status data to single-hop neighbors, mostly containing information about location, speed and identifier; however, it allows for defining new information emerged by need. Example usage of these messages can be in traffic efficiency applications such as remote vehicle monitoring, which gathers periodic status data from vehicles, or cooperative applications such as platooning, which requires kinematic information about surrounding vehicles for decision making. We refer to [53], for detailed information regarding message format specification. 


\subsubsection{DENM}

Decentralized Environmental Notification Message (DENM) [54] is an eventtriggered Facilities layer message that is mainly used by ITS applications to notify other vehicles regarding a detected event. An event is characterized by its identifier (specifying the type of the event), its position, detection time (specifying the expiration time of the event), the destination area (specifying the geographical area over which the DENM message needs to be disseminated, and a transmission frequency. DENM messages are often broadcast over multiple hops to cover the dissemination area. The reliable delivery of these messages to the intended recipients is a key performance requirement due to the importance of the message content. DENM notifications are mainly used in safety critical applications such as collision and accident warnings. We refer to [54], for detailed information regarding message format specification.

\subsection{Data forwarding in vehicular networks}

Data forwarding, as part of a routing protocol, refers to sending data to nodes of a network and is among the main design principles of vehicular communication systems [12]. Four main types of data forwarding can be identified in a vehicular networking system, as Geographical Unicast, Topologically-Scoped Broadcast (TSB), Geographical Broadcast, and Geographical Anycast, elaborated below.

They are Geographical Unicast (i.e., direct or multihop unidirectional transport of data from a single node to a single node using geographic addresses); TSB (i.e., transport of data packets from a single node to all nodes of a vehicular network); Geographical Broadcast (i.e., transport of data packets from a single node to all nodes in a geographical region), and Geographical Anycast (i.e., transport of data packets from a single node to any of the nodes in a geographical region).

\subsubsection{Geographical Unicast}

Geographical Unicast refers to direct or multihop unidirectional transport of data from a single node to a single node using addresses that include node identifier, geographical position, and time information (See Figure 2.7) [12]. 


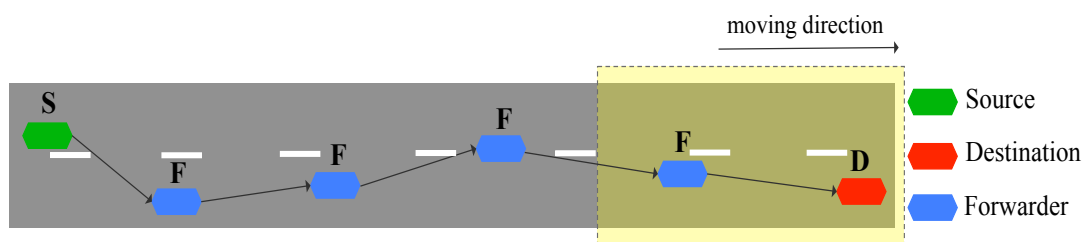

Figure 2.7: Geographic unicast.

\subsubsection{Geographical Anycast}

Geographical anycast refers to transport of data from a single node to any of the nodes located within the target geographical area, specified by a geometric shape (e.g., circle and rectangle). Hence, upon reception of data by at least one of the nodes located within that area, the packet is no longer forwarded (See Figure 2.8) [12].

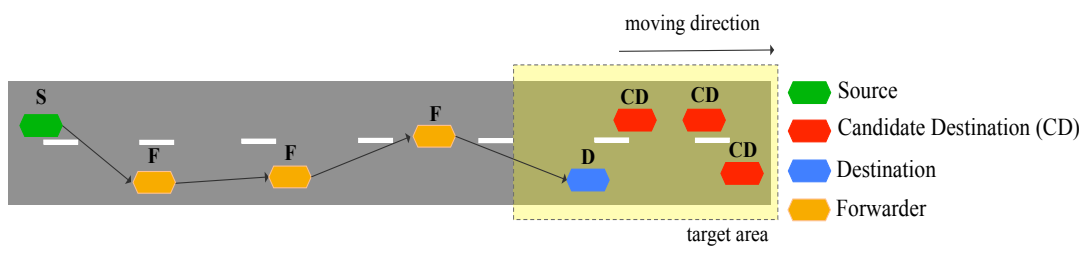

Figure 2.8: Geographic anycast.

\subsubsection{Broadcast}

\section{Topologically-scoped broadcast}

Topologically-scoped broadcast refers to transport of data from a single node to all other nodes within the scope of a vehicular network, over multiple hops (See Figure 2.9) [12].

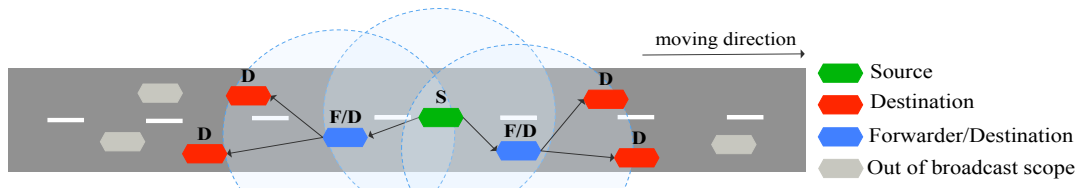

Figure 2.9: Topologic broadcast, with 2 hop coverage scope. 


\subsection{PERFORMANCE REQUIREMENTS AND DESIGN}

CHALLENGES

\section{Geographically-scoped broadcast}

Geographically-scoped broadcast refers to transport of data from a single node to all other nodes within the target geographical area (See Figure 2.10) [12].

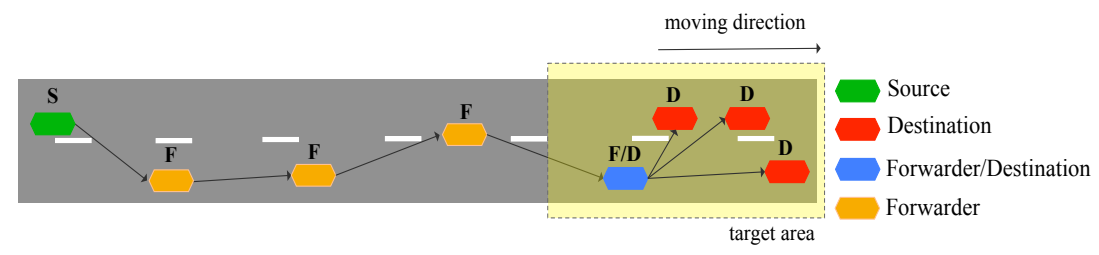

Figure 2.10: Geographic broadcast.

Note that in case of the source node located outside the target destination area, data must be forwarded towards that area by means of a geographic routing protocol.

Several approaches have been proposed in the literature

\subsection{Performance requirements and design chal- lenges}

Vehicular networking is a rather new field of research and development with challenges that must be addressed in order to provide high performance ITS applications. Not all these challenges are relevant to the scope of this thesis; However, here we briefly discuss some of the main ones as a means to gain a general insight regarding the field of Vehicular Networking. For extensive discussion regarding performance requirements and design challenges of vehicular networks, we refer to [44] [27].

\subsubsection{Reliability}

Reliability of vehicular communications is a major performance concern for ITS applications. It refers to correct delivery of data to the intended receivers within reasonable time; However, it is a challenging task to assure reliability of data dissemination in vehicular networks, mainly due to the unstable links of varying network topologies and colliding transmissions. Hence, it is essential employing efficient reliability assurance schemes to provide some measures of reliability guarantee.

Various layers of the communication protocol suite can be involved in the process of improving communication reliability and several techniques have been 
proposed to address this performance requirement in wireless communication networks. At the Physical layer, IEEE 802.11p employs convolutional Forward Error Correction (FEC) [88] [113] [74] channel coding. FEC is a service used to enhance reliability of data transmission at the cost of higher bandwidth consumption, due to using error correction codes. Hence, it is not spectrally efficient, incurring (redundant) overhead even in the absence of errors in the link.

The Data Link layer might provide the means to detect and possibly recover from errors that may occur at the Physical layer. As mentioned earlier in Chapter 1, IEEE 802.11p is an extension of the IEEE 802.11 WLAN technology, retaining the Data Link layer and accordingly the Automatic Repeat Query (ARQ) [88] [113] [74] based error control mechanism of WLAN. ARQ is an error recovery method of the Data Link or the Transport layer, using acknowledgments to achieve reliable data transmission. This messages incur transmission overhead. ARQ is only applicable to unicast type of communication which is still questionable its performance due to the head of line blocking [82].

By means of error detection codes, the Transport layer can verify data integrity and inform the sender of data by sending ACK messages accordingly. This is not a suitable alternative for improving reliability of vehicular broadcast communication, as the limited medium would be occupied by loads of ACK messages, sent by individual recipients of data, which also gives rise to collisions and loss of these messages. At the Network layer, design of efficient routing protocols can contribute to dissemination reliability, in an implicit manner, via a dependable and resilient data forwarding mechanism; However, as long as the sender is not explicitly notified regarding delivery of data, it is an open challenge ensuring transmission reliability.

Error recovery for the provisioning of a reliable service can be handled also at the Application layer. Effective error recovery by the lower layers may require fundamental changes in the standardized protocols. While an error recovery mechanism operating at the Application layer can work with already deployed network. In general, a well designed system that considers combinations of appropriate reliability settings at different protocol layers (i.e., cross-layer error recovery) may result in optimized resource utilization and user experience.

While approaches based on blind retransmissions of data or ACK messages burden the network with traffic overhead, other approaches of reliability improvement may lack in providing to the sender explicit information regarding packet delivery to the intended recipients. In general, reliability improvement mechanisms incur some overhead to the network and as a result function more slowly and with less scalability. This often is not an issue for unicast, but it may become a bottleneck for multicast and broadcast types of communication. Most safety messages in vehicular networks are broadcast based, demanding reliable delivery. Hence, it gains significant importance ensuring end-to-end re- 


\subsection{PERFORMANCE REQUIREMENTS AND DESIGN CHALLENGES}

liable delivery of broadcast data and is considered as an open research challenge in the design and deployment of VANET. Efforts towards this objective have resulted in a number of works in this regard and we will refer to them in the corresponding chapters of this thesis. For further insight regarding reliability aspect of vehicular communication, we refer to [38] [55].

\subsubsection{Scalability}

Scalability is a crucial characteristic for large and distributed systems. It is defined for a network as being operable given varying densities of nodes without suffering a significant decrease in performance or increase in complexity [102]. In a vehicular network, the scalability issue may arise in different contexts and it depends to many factors. The number of active vehicles and applications in a vehicular network and protocol design (e.g., medium access and routing protocols) are example factors influencing scalability of a vehicular network.

Scarcity of the wireless medium is a major scalability bottleneck. As already mentioned in Chapter 1, medium access of the IEEE $802.11 \mathrm{p}$ standard is based on the CSMA/CA from EDCA which is a contention-based protocol. This results in significant decrease of throughput at high network loads whereas a maximum latency cannot be guaranteed, due to long contention periods to access the medium.

High mobility of vehicles results in frequent communication link breakage and accordingly changes in topology and vehicle positions. This demands a quick response from a routing protocol, otherwise the routing information would be outdated. This in turn leads to excessive routing overhead and accordingly, the routing protocol cannot scale well with varying network size and topology. Design of an efficient routing protocol not only results in better scalability, but also contributes to a higher level of dissemination reliability by improving the packet delivery ratio via a resilient data forwarding mechanism. Several works have been proposed in the literature regarding design of routing protocols suitable for various scenarios of vehicular communications. For further detail we refer to [117] [46].

\subsubsection{Security}

To become a real technology that can guarantee proper functioning of ITS systems, vehicular networks need to be integrated with a strong and practical security mechanism to protect them against security attacks. Otherwise, vehicular networks may provide means for malicious use. This is of critical importance particularly for safety applications, where an attack may lead to life-threatening 
consequences. Further, unsecure vehicular systems will be obstacles to market penetration and large-scale deployment.

Security in a vehicular network can be specified by the following requirements. Message authentication allowing the receiver of data acknowledge the sender. Message integrity, referring to protection of data against alteration. Message non-repudiation, making the sender cannot deny having sent the message, enabling accountability. Message confidentiality, protecting the content of a message from unauthorized access. Access control, referring to authorization of users for the type of tasks that they are allowed to carry out. User privacy, keeping personal user information untraceable [105].

There are currently a number of techniques, proposed for addressing the above-mentioned requirements and accordingly securing vehicular communication. Digital signatures are primary tools for message authentication and are usually implemented via a Public Key Infrastructure (PKI), based on asymmetric cryptography. That is, the sender signs the message using its private key, assigned by a Certification Authority (CA), and attaches a certificate including a public key (also assigned by the $\mathrm{CA}$ ). Upon reception, the receiver validates the certificate and accordingly decrypts the message using sender's public key. This procedure ensures the integrity and authenticity of the message will be verifiable by the receiver. Additionally, digital signatures provide non-repudiation protection and as a result impersonation attacks cannot be launched anymore. Vehicles' privacy can be ensured by each vehicle having a pool of keys and using different ones to sign each message. This avoids linking up a vehicle with its key.

The main constraint of using digital signatures and certificates is the overhead they add to the system, by making messages of significantly larger size. Detailed discussion of security aspect of vehicular networks is out of scope of this thesis. We refer to [103] [99] [98] [47] for more information regarding security challenges and techniques to address them.

\subsection{Evaluation tools}

Though field operational evaluation of vehicular networking scenarios can provide realistic outcomes, it is a demanding task to fulfill and can be prohibitively expensive, in particular for large-scale deployments. Accordingly, other alternative evaluation tools and methodologies have been developed by need. Such tools are meant for rather straight forward and efficient evaluation of vehicular networking scenarios, without the need for real-world implementation, though each has its own challenges and strengths and weaknesses. Models can represent systems at various levels of detail depending upon their application and 
required accuracy. It is important the choice of the appropriate tool and modeling approach, influencing quality of performance evaluation. In what follows, we briefly introduce the two mainly used methods for evaluating performance of vehicular networking scenarios.

\subsubsection{Simulations}

Simulation is a widely used method for evaluating performance of various networking scenarios. The principal idea is modeling behavior of a network, where either the interaction between different network components is calculated using mathematical formulas or it is actually captured and utilized the experimental observations from a real network. Accordingly, various attributes of the network can be modified in a controlled manner and the corresponding results can be analyzed to assess how the network would behave under different conditions.

Simulation for vehicular networks encompasses simulators for mobility of vehicles (i.e., vehicular traffic flow) and wireless communication between them. Depending on the application of consideration, different simulators for vehicular traffic can be used which are categorized into microscopic, mesoscopic and microscopic, based on the level of details describing traffic flows [139]. Microscopic models include the highest level of details by individually considering each vehicle's behavior (e.g., car-following and lane-changing). Hence, the dynamic variables of the models represent microscopic properties such as the position and velocity of single vehicles. Accordingly, they have the ability to take into account the complex interactions among vehicles and also road infrastructures, at the cost of being computationally more expensive. SUMO [10], VanetMobiSim [11] [70], VISSIM [58], GMSF [13], CORSIM [69], TRANSIMS [121] and Quadstone Paramics Modeller [122] are examples of urban microscopic traffic flow simulators. Macroscopic models have a higher level of abstraction and aggregate vehicles by treating vehicular traffic according to fluid dynamics. They formulate relationships among traffic flow characteristics such as density, flow and mean velocity of a traffic stream. Such models are usually obtained by integrating microscopic traffic flow models and then translating the single-entity level characteristics into analogous system level characteristics. VISUM [9], CUBE [1] and SATURN [138] are examples of macroscopic traffic flow simulators. Mesoscopic traffic flow models are developed to fill the gap between microscopic and macroscopic levels of modeling. They describe vehicle behavior in aggregate terms such as probability distributions. Aimsun [14] is a simulation package that integrates mesoscopic and microscopic simulators. For more detail regarding traffic flow models and simulation tools, we refer to [139].

Network Simulator 2 (NS-2) [6], Network Simulator 3 (NS-3) [7] [111], Qualnet [5], OPNET [4], OMNeT++ [3] and JiST/SWANS [2] are examples of simu- 
lators used for wireless network communications. Network simulators comprise a wide range of networking technologies and protocols in order to build complex networks from basic network components like nodes, routers and links.

Most network simulators (including all the mentioned examples) are based on discrete-event simulation in which the system state is discrete (i.e., discrete state space) and changes at particular point in time and remains in that state for some time.

There are simulation frameworks combining simulators for vehicular traffic and networking. Interactions between traffic and network simulators is done via standardized interfaces. Veins [8] is an example that combines the open source network simulator OMNET ++ and the open source traffic simulator SUMO.

\subsubsection{Analytical modeling}

Even though simulations are efficient tools to model and evaluate performance of a real world system, and offer great versatility in analyzing complex systems, they have limitations, namely in terms of time, repeatability and additional analysis options. For instance,they could be error-prone in case of limited number of simulations. But on the other hand, performing a large number of simulations can be extremely time-consuming. Also, compared to analytical methods, simulations offer a small range of calculation results for performance indicators.

Analytical modeling refers to presenting functionality of a system and interrelations between its components in terms of mathematical expressions. Whereby, it enables quantifiable analysis of a system's parameters by relying on mathematical concepts and principles and accordingly obtain closed-form expressions for a wide range of measures, mostly not feasible via simulations. Most importantly, analytical modeling provides insight on performance improvement. That is, optimization techniques can be utilized in order to enhance system performance at the design phase. By translating application performance requirement into system design and resource requirements, analytical modeling provides the means to investigate and improve a system's capacity to fulfill such a requirement. Further, trade-offs between various system parameters and performance measures can be acquired.

Analytical models can serve different purposes and depending on the objective of modeling, different levels of information and complexity in system characterization must be entailed into the model. Analytical models may be used to gain an understanding of the current state of a system and to measure its performance. Analytical models can also be used for prediction of a system's functionality by introducing a series of "whatif" changes to the environment (e.g., workload volume increase, addition of new users, etc). This allows to tune the system for improved performance. Some may use analytical models 
as inputs to a second model so as to measure the effects of the combination of two existing systems. Analytical models have also the potential to be extended by including more details into a system definition and gain more observations regarding system performance, as per need.

By supporting parametric analysis, analytical models provide a general means for performance evaluation in a sense that they can take in various input, while simulation results are based on an already defined scenario. This allows efficient evaluation of various system design alternatives by providing means to quickly quantify performance measures against various input settings. 



\section{Reliable V2V broadcast: single-hop communication scenario}

V2V single-hop broadcast is a common form of dissemination for many application types, demanding small-scale neighborhood awareness (e.g., lane change assistance, collision warning). In this context, dissemination reliability, as a key performance factor, comes into question. In this chapter, we propose a reliability mechanism for end-to-end message delivery assurance and analyze its functionality in a single-hop communication setting. This receiver-based mechanism aims at fulfilling the received message integrity yet keeping the overhead at a reasonably low level. Using an analytical analysis based on absorbing Markov modeling, we analyze its performance in terms of relevant indicators, such as reliability overhead and delivery ratio. The analytical results are validated by simulations carried out in the NS-3 simulation environment. The analysis shows that in a single-hop setting, this mechanism provides good reliability, while giving little overhead for high number of receivers. Publications related to this chapter are [65, 66].

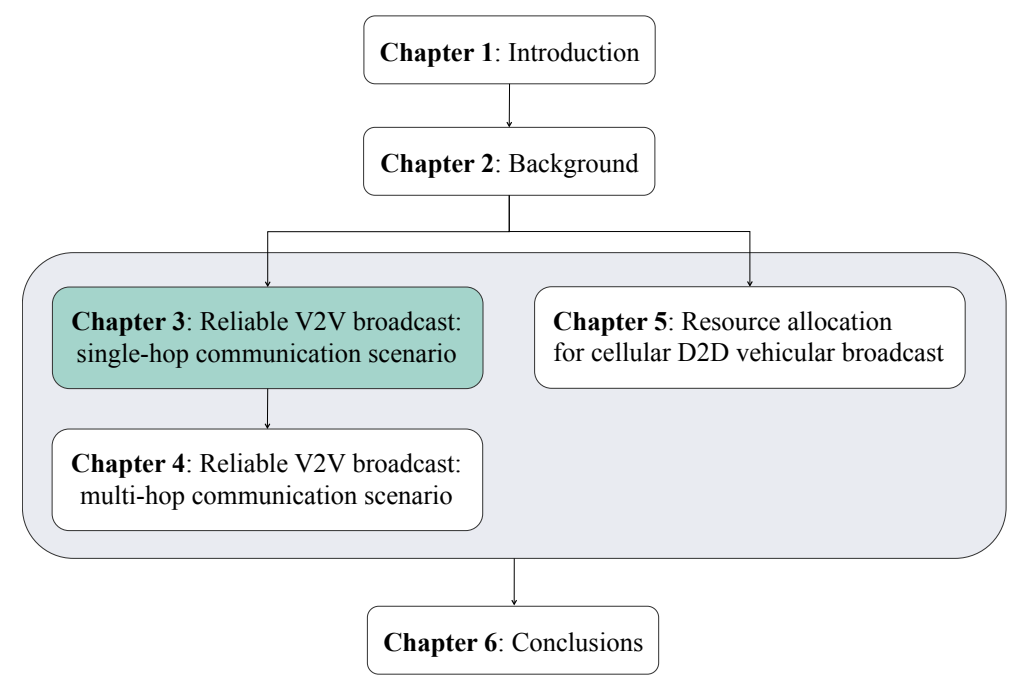


This chapter is organized as follows:

- Section 3.1 gives an introduction to vehicular broadcasting and states the contribution of this chapter.

- Section 3.2 provides a brief account of the related work on single-hop vehicular broadcasting and its challenge of reliability.

- Section 3.3 introduces an glse2e reliability assurance mechanism for vehicular broadcasting.

- Section 3.4 presents our approach of modeling the proposed mechanism, including the modeling assumptions and parameterization in Section 3.4.1 and Section 3.4.2, respectively.

- Section 3.5 is devoted to performance analysis of the proposed mechanism, by first introducing the relevant metrics in Section 3.5.1 and afterwards presenting the obtained results of analytical modeling and validation by simulations in Section 3.5.2.

- Section 3.6 highlights main conclusions of this chapter. 


\subsection{Introduction}

The transport protocol of a vehicular communication system must meet application requirements, which, in turn, affects its design principles [12]. In our study, we consider reliability as one of the most substantial requirements. Reliability refers to assuring that a message has reached the highest possible number of intended receivers. Due to the inherently unreliable nature of vehicular environments, it is very challenging to design an end-to-end reliable (geo-)broadcast protocol guaranteeing delivery of packets to all intended receivers of a vehicular network. For instance, lack of a couple of packets from the set of packets constituting big messages such as maps, at the receiver-side, makes the whole message useless.

Providing E2E reliable delivery for (geo-)broadcast is an open area of research. In this chapter, we propose a receiver-based E2E reliability assurance mechanism in the context of geographically scoped broadcast in vehicular networks. According to this mechanism, every packet, as a part of a message, is marked with a sequence number, in an increasing order. By sequence checking, the missing packets of a message are detected at the receiver-side and requested afterwards from the neighbors by means of broadcast. Those neighbors having these packets in possession reply by broadcasting them. In order to minimize the effect of collisions as a result of concurrent requests and replies, a back-off timer-based policy is introduced. The proposed approach does not target a specific application but could be applied in scenarios featuring large (e.g., maps) and small (e.g., regular awareness) sized messages. 
The main contributions of this chapter are as follows:

- we propose a mechanism for E2E reliability assurance for vehicular (geo-)broadcast and derive an analytical modeling of its functionality in a single-hop communication scenario;

- we provide fundamental insight into the behavior of proposed mechanism and quantify its performance using the analytical model, in terms of relevant measures;

- we validate observations from the analytical analysis, using simulations.

\subsection{Related work}

There are plenty of ITS applications demanding to address a group of vehicles falling into the same geographic location for a variety of purposes. Geocasting is the routing protocol fitting to this demand. However, unreliability of vehicular environments makes it quite challenging to guarantee E2E (geo-)broadcast reliability, which could be of significant importance depending on the application type (e.g., safety applications). Various mechanisms for (geo-)broadcasting and proposals introducing reliability to the scope have emerged in the literature. In general, (geo-)broadcasting reliability approaches could be divided into two main categories as multi-hop and one-hop and further into sender-oriented (i.e., the recovery action is initiated by the sender such as regular repetitions, also known as implicit recovery) and receiver-oriented (i.e., the recovery action is initiated by the receiver, such as negative acknowledgments (NACK), also known as explicit recovery) approaches. Here, we refer to those mechanisms and protocols related to our approach.

In [90] and [93], overhearing of rebroadcasted messages is considered as an implicit acknowledgement for the sender and all the vehicles with pending rebroadcasts. Preferred and Contention Based Forwarding (PCBF) [110] approach relies on overhearing the message rebroadcast as well, as an implicit acknowledgement. In order to avoid redundant rebroadcasts, a kind of explicit acknowledgment is considered in this scheme. That is, if the most recent forwarder hears rebroadcast of the packet, it sends an explicit acknowledgement to the former forwarder to stop further unnecessary rebroadcasts. In Distributed Robust Geocast (DRG) [80] while distance-based timers are applied to rebroadcast, overhearing of rebroadcasts is considered as an implicit acknowledgement. In this approach, two kinds of retransmissions as short interval rebroadcasts and long interval rebroadcasts are considered to cope with communication losses and network fragmentation, respectively. The MQ-MapCast [115] and the approach 
by $\mathrm{C}$. Wu et al. [152] are also based on rebroadcast overhearing as implicit acknowledgements. IVG [33] and UV-CAST [140], both are overhearing based suppression schemes with rebroadcast back-off timers, inversely proportional to the distance between the vehicles and the sender, to ensure longer packet traverse distances. The UGAD [26] geocasting protocol is also a timer-based rebroadcast approach with the difference at back-off time assign criteria, which is preferential for the vehicles at intersections. Such schemes in general improve hop-by-hop and not E2E traverse reliability with the cost of increased overhead and redundancy.

Reliable Vehicular Geo-broadcast (RVG) [83] is based on Slotted Restricted Mobility Based (SRMB) scheme and Pseudo- Acknowledgments (PACK) scheme. While SRMB is responsible for data dissemination over a specified distance, PACK assures multi-hop dissemination reliability by interpreting overheard rebroadcasts as a measure of successful transmission. When the neighbor elimination [127] [126] and the multipoint relaying [108] schemes of SRMB fail, Pseudo-ACKnowledgement (PACK) scheme detects unacknowledged links and repeats forwarding. In [94] besides the distance between the current receiver and the previous forwarder, the number of the local copies of the message at each receiving vehicle, is considered to take the rebroadcast decision. This approach is based on acknowledging the received messages to assure reliable delivery by indexing the copy of the messages. In [120] an end-to-end geocast acknowledgement scheme is proposed, in which individual acknowledgments are accumulated into larger messages in an aggregator. The acknowledgement is later sent to the source using a sink tree and position update schemes in order to find the path back towards the source. Acknowledged Broadcast from Static to Highly Mobile (ABSM) [112] approach is based on the Connected Dominant Set (CDS) scheme [127] [126] and Neighbor Elimination Scheme (NES) for reliable data dissemination. Vehicles possessing the message of interest set a back-off timer, such that the vehicles in the CDS choose a shorter back-off timer, and rebroadcasts it upon timer expiration if discovering their neighborhood is not fully covered. Identifiers of the recently received messages are included in beacons as acknowledgments. In Adaptive and Reliable Broadcast (ARB) [159], each sender sets a receive acknowledgement window, which is divided into many adaptive slots proportional to the number of neighbors. The retransmission will be started if any acknowledgement from a neighbor in the table of neighbors is missing upon expiration of the acknowledgement window. MHARQ-SC [41] is an ACK-based approach such that a couple of packets to be transmitted are combined at first based on an Symbol Combining (SC) algorithm and then transmitted to the intended receivers. It is assumed to deploy an error detection code at the receiver end able to detect any loss pattern and send a negative ACK to the sender side, otherwise a positive ACK will be sent. In [87] a distributed channel usage 
scheme is used between TV White Space (TVWS), for message dissemination, and DSRC for control. The On-Demand Recovery Algorithm (ODRA) is applied to recover losses from decoding such that, the vehicle unable to decode broadcasts a negative acknowledgement in the DSRC channel. The vehicles possessing the message unicast it in the DSRC channel. Reception failures by TVWS rendezvous errors are recovered in an indirect way by overhearing of retransmissions in the DSRC interface. However, if the vehicle does not overhear retransmission of any missing packet, will not be able to distinguish it.

DEEP [43] and the approach in [86] both propose a repetition-based scheme for increase reliability assurance, while keeping it efficient by computing efficient rebroadcasting intervals based on the vehicle speed, transmission range and the number of the planned repetitions. In Distributed Vehicular BroadCAST (DVCAST) protocol [131] rebroadcast decision is based on neighbor detection. That is, upon reception of a packet by a vehicle, if it is connected to its vicinity, it is assumed that most probably the neighbors have also received the same packet and a broadcast suppression mechanism [149] is applied afterwards. However, if the vehicle is sparsely connected, it can immediately rebroadcast the received packet. If the vehicle is totally disconnected, it stores the packet till it expires and then rebroadcast it once connected again. GeoSUZ [28] proposes to divide the geobroadcast target area into sub-areas and to use different message dissemination techniques in each of them to enhance reliability. Still, E2E transfer may not be satisfied. SmartGeocast [158] encompasses two phases as initialization and maintenance. The latter is considered for reliability improvement by keeping rebroadcasting at regular time intervals, within a smaller area than the target area to be less redundant and cost-efficient. As a result, this approach fails to guarantee E2E reliability for all the intended receivers. The approach in [144] proposed XOR coding for loss recovery in vehicular communications such that after original transmission, instead of repeating the packet transmission for a couple of times, the sender XORs its packet with other packets received from other vehicles and repeatedly transmits the XORed packet, while there is no explicit information from the receiver side for such retransmissions.

In this work, we propose an on-demand reliability assurance approach for (geo-)broadcast, in which receivers are able to detect lost packets and explicitly ask for them.

\subsection{End-to-End reliability mechanism}

In this section we introduce an $\mathrm{E} 2 \mathrm{E}$ reliability assurance mechanism for (geo-)broadcast in vehicular environments which we developed in the framework of the European FP7 project MOBILITY2.0 [16]. It is a receiver-based 
approach enabling loss detection and correction by implementing a sequence checking functionality and is suitable for a wide variety of ITS applications demanding strict delivery requirements. Before proceeding with the system description, it is worth pointing out that we assume that the underlying ETSI ITS standard geo-networking infrastructure [50], introduced in Chapter 2, is functionally available, providing the basis to implement such a facility.

The sequence number checker module works as demonstrated in Fig. 3.1 and Fig. 3.2. At first, the packets to be broadcast are sequentially numbered. Further, a unique session identifier is assigned to the packets constituting a single message and flags to identify the first and last packets of a message are set as well. Hence, the receiving side can detect any inconsistency in the sequence of received messages. If any missing packet is detected, a back-off timer randomly set from 0 up to a few milliseconds is started. Upon expiration of this timer, a Single-Hop Broadcast (SHB) query is initiated to fetch the missing packet from any neighbor by encompassing the relevant sequence number in the request. The aim of this random back-off timer is to prevent many duplicate requests from different receivers for the same packet and consequently collisions. During the countdown period, as any received broadcast of that packet cancels the timer, any received query for the same packet causes extension of this pending query, up to limited number of times. Such functionality gives a break to the neighbor with pending request to get the packet of interest as a result of the heard query. If it does not happen, it will broadcast its own query to get that packet. Upon broadcasting the request, the vehicle starts a randomly set back-off timer and waits for the reply. During the countdown process, if the vehicle does not get the reply, it will rebroadcast a request to try once more to get the desired packet. When the source of the packet or any other neighbor already having the packet receives the retransmission request for the packet, it verifies whether it has it in its data cache. If the packet is found, a randomly set back-off timer having value from 0 up to a few milliseconds is started, after which the packet is single-hop broadcasted, if it is not broadcasted by another vehicle before the timer expires. Again, this waiting procedure is designed to prevent numerous duplicate retransmissions and collisions. Note that even by utilizing single-hop broadcast of the request packet, it is of high probability that the packet will finally reach a vehicle possessing the requested packet after some time, because of a predefined number of request broadcasts. 


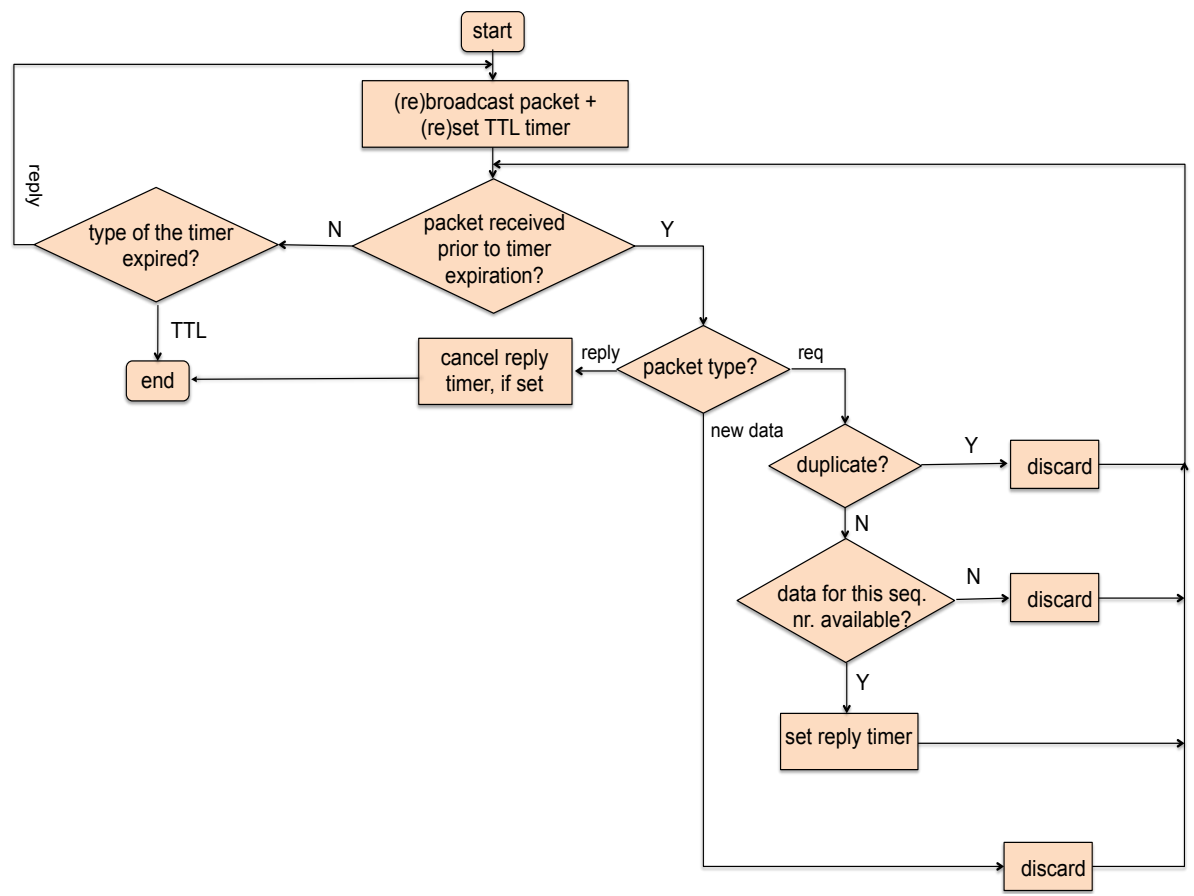

Figure 3.1: The sender-side of the reliability mechanism. 


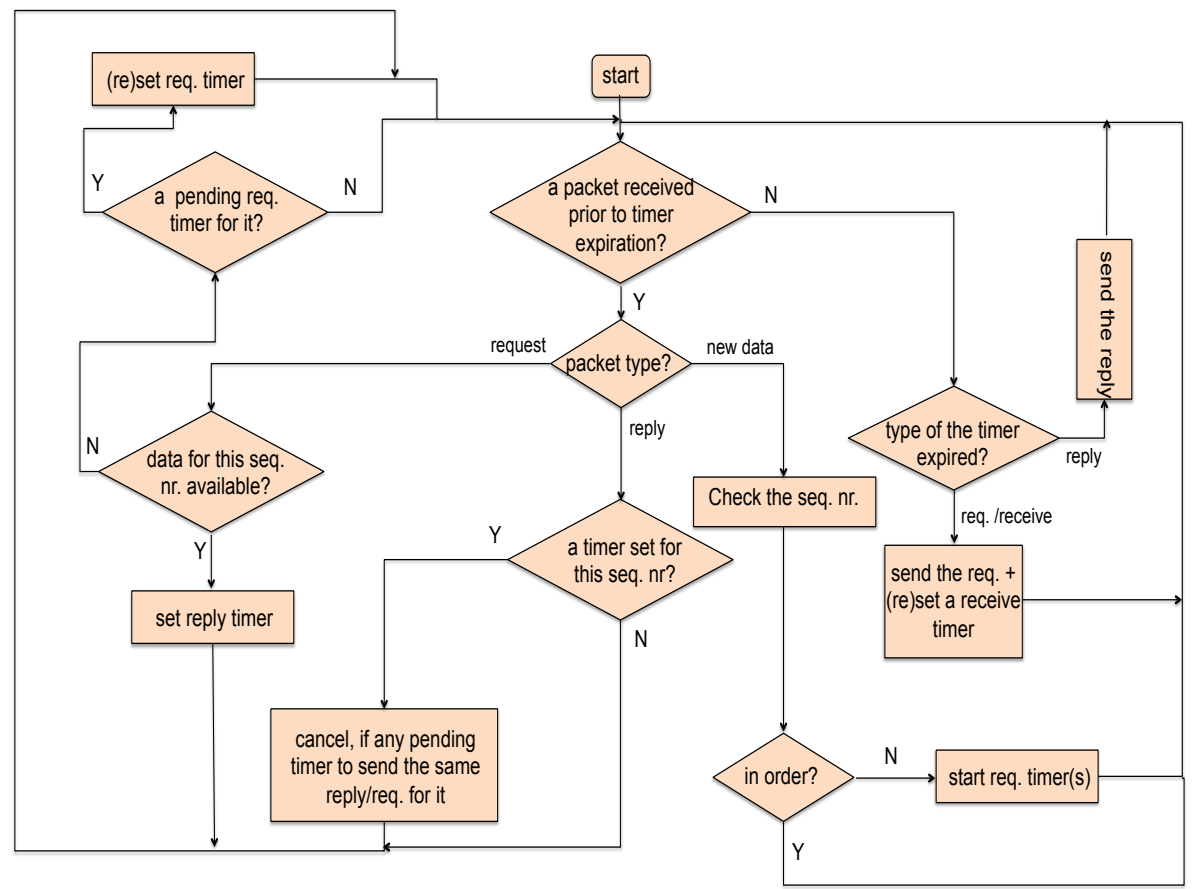

Figure 3.2: The receiver-side of the reliability mechanism. 


\subsection{Modeling approach}

In this section, we present the modeling approach for evaluating the performance of the proposed reliability assurance mechanism. We formulate an analytical model of the proposed mechanism for a single-hop communication setting as shown in the example Figure 3.3, capturing all its important features. The considered performance metrics of interest are those pertaining to delay, loss and overhead performance of the proposed mechanism. Table 3.1 summarizes the notation we use throughout this chapter.

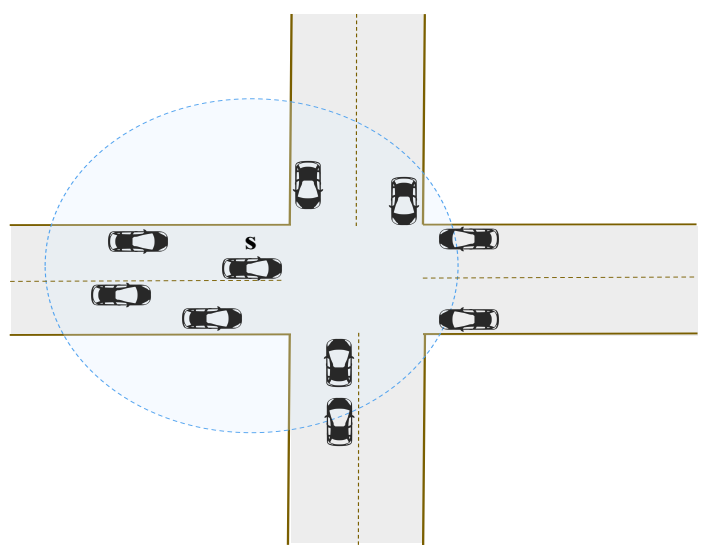

Figure 3.3: Single-hop information broadcast by node " $s "$.

\subsubsection{Considered scenario}

Taking into account all the details of the proposed mechanism makes the modeling effort extremely complicated. Instead, we consider a simplified version of the mechanism taking into account all the important details affecting delay and loss performance. The considered scenario consists of $N$ receivers and an additional station " $s$ " being the source of the broadcast message. All the stations can hear each other, that is, they could successfully receive and transmit with non-zero probability.

The simplified system for performance modeling operates as follows. First, the originator transmits a packet by first choosing a random back-off delay expressed in the number of time slots. As denoted by $T_{\text {slot }}$ in Table 3.1, the time slot is the amount of time prior to transmitting a packet. We assume that the back-off delay is drawn from the geometric distribution with parameter $r_{T}$ (mean $\left.1 / r_{T}\right)$. The probability of data packet loss due to wireless channel conditions is 
Table 3.1: Notation used throughout this chapter.

\begin{tabular}{|l|l|}
\hline Parameter & Definition \\
\hline \hline $\mathrm{N}$ & The number of the receivers \\
\hline $\mathrm{K}$ & The number of the retransmission requests allowed \\
\hline $\mathrm{D}$ & The number of steps till absorption in the Markov chain \\
\hline$T_{t i m e o u t}$ & Constant waiting time to receive the reply after a request (s) \\
\hline$T_{T}$ & Transmission time of a data packet (s) \\
\hline$T_{R}$ & Transmission time of a retransmission request packet (s) \\
\hline$T_{I}$ & The set of all nodes of a network \\
\hline$p_{T}$ & Data packet loss probability \\
\hline$p_{R}$ & Retransmission request packet loss probability \\
\hline $1 / r_{T}$ & Mean number of back-off slots prior sending a data packet \\
\hline $1 / r_{R}$ & Mean number of back-off slots prior sending a request packet \\
\hline$T_{\text {slot }}$ & Duration of a time slot (s) \\
\hline$E\left[T_{i}\right]$ & Mean time spent in the state $i$ of the chain (s) \\
\hline$E[T]$ & Mean time spent in transient set of states till absorption (s) \\
\hline$E\left[T_{i} \mid F\right]$ & Mean time spent in the state $i$, conditioned on failure (s) \\
\hline
\end{tabular}

considered to be independent and the same for all the stations and equal to $p_{T}$. This assumption is realistic for rather dense communities. If say, $i$ out of $N$ stations do not receive the packet correctly they will all choose a certain back-off value from the geometric distribution with parameter $r_{R}$ independently of each other to broadcast a retransmission request for the lost packet. The detection of packet loss is based on checking the sequence number of the received packets. Requesting stations then start a retransmission timer and wait to receive the packet of interest. Upon expiry of the timer, they will resend the request, only if it is not yet received. If no collision happens as a result of concurrent transmission of requests, the request for the packet is received correctly at each of the $(N-i)$ stations having the original data packet, with probability $1-p_{R}$. Here, again it is assumed that the request packet loss probability due to wireless channel conditions is the same for all the stations. Assuming that $j$ stations out of $N-i$ have received the request, they all will choose a certain back-off value 
from a geometric distribution with parameter $r_{T}$ to reply with the packet of interest. Subsequently, this packet of interest is correctly received at each of the $i$ stations independently with probability $1-p_{T}$. The procedure repeats itself until all the stations have received the data packet correctly or the number of allowed retransmission requests $K$, is exceeded.

In this study we assume that the collision probability is negligible compared to the probability of incorrect reception of a packet. In other words, performance degradation is mainly caused by incorrect reception of packets at the air interface. This is the case when the number of stations involved in packet distribution is rather limited and/or the contention window interval is large. Even in the last case the size of data packet is usually much larger than the maximum possible contention window size. We will however take into account the delay caused by back-off times in delay analysis and discuss how to incorporate the effect of collisions to the proposed model at the end of the chapter.

\subsubsection{Modeling and parameterization}

In this section we introduce our approach of analytically modeling the proposed reliability assurance mechanism which is based on the concept of absorbing Markov chain. For this reason we first briefly remind the basics of absorbing Markov chain theory [77].

A finite-state Markov chain is called absorbing if it contains one or more states that, once entered, cannot be left. The states of an absorbing chain that are accessible from any other states, except for absorbing ones, are called transient. A chain is said to be time-homogeneous if state transition probabilities depend on elapsed time, not absolute time. Homogeneous absorbing chains are suitable to model the processes that eventually stop on one or few states, i.e., absorb.

Recalling the operation specification of the proposed mechanism, we observe that we can represent the process of packet distribution from the source " $s$ " to all $N$ recipients using the discrete-time homogeneous absorbing Markov chain with the state space $\{S(n), n=0,1, \ldots\}$, where the state number, $S(n) \in\{0,1, \ldots, N\}$, describes the number of stations not yet having the packet of interest by time $n$. It is important to note that here we are dealing with a Markov chain embedded at the moments of state changes (i.e., transitions). $S(n)$ refers to the state of the system after the $(n-1)^{t h}$ retransmission request plus associated retransmission, or, if one of these got lost, after expiry of the corresponding timer. Furthermore, $S(0)$ is the initial system state, and $S(1)$ the state after the initial transmission. In other words, time intervals between state changes are not constant and depend on the protocol operation. 
The proposed model behaves as follows. Initially, the model is in the state $S(0)=N$ representing the situation when none of the stations received the packet yet. The source of the message performs the original transmission so that 0 or more of the stations out of $N$ receive the packet correctly. Once it happens, the model enters one of the states out of the set $S(1)=\{0,1, \ldots, N\}$ with the only absorbing state 0 . If the chain is not immediately absorbed in the state 0 it jumps between transient states as a result of retransmission requests and replies and finally gets absorbed in the state 0 , where all the stations have the packet of interest. Since the number of stations having the packet after the next retransmission attempt depends only on the number of stations having it right now, the process is Markovian in nature and can be modeled using the discrete-time homogeneous Markov chain, as described in what follows. A Markov chain with $N$ transient states and one absorbing state is characterized by a $(N+1) \times(N+1)$ transition matrix $T[77]$ as

$$
T=\left(\begin{array}{cccccc}
q_{N, N} & \ldots & q_{N, 3} & q_{N, 2} & q_{N, 1} & q_{N, 0} \\
\vdots & \ddots & \vdots & \vdots & \vdots & \vdots \\
0 & \cdots & q_{33} & q_{32} & q_{31} & q_{30} \\
0 & \cdots & 0 & q_{22} & q_{21} & q_{20} \\
0 & \cdots & 0 & 0 & q_{11} & q_{10} \\
0 & \cdots & 0 & 0 & 0 & 1
\end{array}\right)
$$

that can be written in the compact form as

$$
T=\left[\begin{array}{cc}
Q & \vec{q} \\
\overrightarrow{0} & I_{1}
\end{array}\right]
$$

where $Q$ is an $N \times N$ matrix whose elements, $\left\{q_{i j} \mid i, j=1,2, \ldots, N\right\}$ are transition probabilities between the transient states of the absorbing Markov chain, as shown in Figure 3.4 . 


$$
Q=\left(\begin{array}{ccccc}
q_{N, N} & \ldots & q_{N, 3} & q_{N, 2} & q_{N, 1} \\
\vdots & \ddots & \vdots & \vdots & \vdots \\
0 & \ldots & q_{33} & q_{32} & q_{31} \\
0 & \cdots & 0 & q_{22} & q_{21} \\
0 & \cdots & 0 & 0 & q_{11}
\end{array}\right)
$$

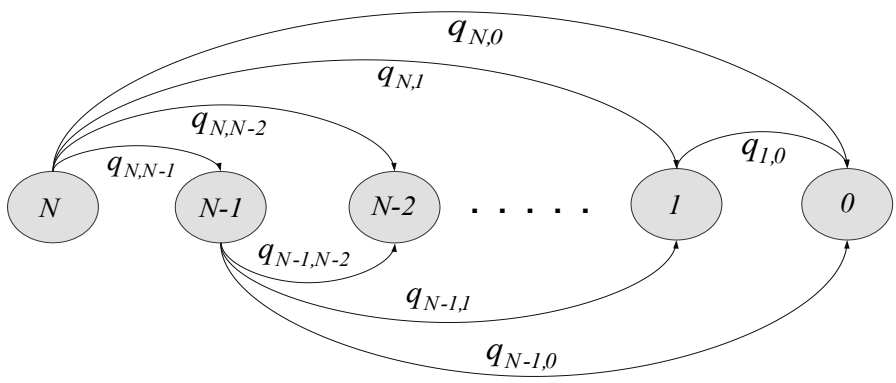

Figure 3.4: Transition probabilities between states of an absorbing Markov chain, with $\mathrm{N}$ transient and one absorbing states.

$\overrightarrow{0^{T}}$ is the vector of zeros of size $1 \times N$ and $\vec{q}$ of size $1 \times N$ is the vector containing transitions from the transient states $\{1,2, \ldots, N\}$ to the absorbing state 0 as follows.

$$
\vec{q}=\left(q_{10}, q_{20}, \ldots, q_{N 0}\right)
$$

We could reform the canonical form of the Markov chain in Eq. 3.2 into the following form

$$
q_{i 0}+\sum_{j=1}^{N} q_{i j}=1, \quad i=1,2, \ldots, N
$$


where $q_{i 0}$ is the probability of going to the absorbing state, directly from the state $i$. Introducing $\vec{e}$ as a vector of ones of size $1 \times N$, in matrix form we have

$$
\vec{q}+Q \vec{e}=1
$$

showing that the model is characterized by $Q$. We also need the initial state distribution vector $\vec{h}$, which is the probability distribution of the number of nodes having the packet after original broadcast by the sender, to fully characterize the model. To parameterize the proposed model we should provide the $N \times N$ transition probability matrix $Q$, and $\vec{h}$ a $1 \times(N+1)$ vector $\left(h_{0}, h_{1}, \ldots, h_{N}\right)$ providing the initial distribution of states of the model.

We choose to define the initial state distributions at state $S(0)$ and $S(1)$ explicitly, as the state transition probabilities in $S(0)$ are different from those in the other states. Clearly, $S(0)=N$, as none of the receivers will have the packet initially. Further, we define the state probability distribution at $S(1)$ as $\vec{h}$. This initial state distribution of the system is given by the number of stations successfully receiving the packet of interest at the first transmission attempt. Let $h_{i}$ denote the probability of residing in state $i, 0 \leq i \leq N$ after the original transmission, which means exactly $i$ stations fail to receive the packet correctly, while $N-i$ succeeded to receive the packet. Hence, recalling that $p_{T}$ is the probability of incorrect reception, $h_{i}$ is derived as

$$
h_{i}=\left(\begin{array}{c}
N \\
i
\end{array}\right) p_{T}^{i}\left(1-p_{T}\right)^{(N-i)}, i=0,1, \ldots, N
$$

which follows a binomial distribution with parameters $p_{T}$ and $N$. From now on we will use the notation $B(N, p, i)$ to denote binomial probabilities. The probability of residing in state $i, 0 \leq i \leq N$ after $k$ retransmission requests is given by $i^{\text {th }}$ element $\left(P_{i}^{k}\right)$ of vector

$$
P^{k}=\vec{h} \cdot T^{k}=\left[P_{0}^{k}, P_{1}^{k}, \ldots, P_{N}^{k}\right]
$$

In order to find $q_{i j}, i, j=1,2, \ldots, N$ we need to consider how our system evolves starting from an arbitrary state $i$. The state transition diagram for arbitrary 
state $i, i=1,2, \ldots, N$ is illustrated in Fig. 3.5. Note that the states drawn with dashed lines are the so-called "phantom" states that do not belong to the model and are shown for illustrational purposes only. The number in the phantom state represents the number of stations already having the packet that have received a retransmission request. The only transitions allowed from the state $i$ are those leading to smaller values associated with states, i.e., $j=0,1, \ldots, i$. The actual probabilities of transitions depend on the probabilities of incorrect reception of data and retransmission request packets, denoted by $p_{T}$ and $p_{R}$, respectively. On top of this when $i<N$ there is competition for resources when either a retransmission request or a data packet is sent. Assume that the system

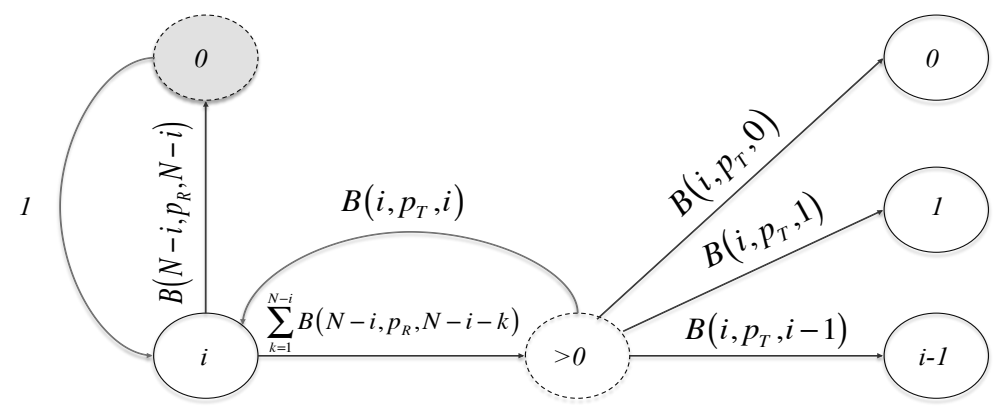

Figure 3.5: Transitions from state $i$ of the the absorbing Markov chain.

is in the arbitrary state $i$ at the time $n$, that is, $N-i$ stations have successfully received a packet so far in the first " $n$ " steps. Consider the probability, $p_{\text {success }}$, that a retransmission request for a missing data packet is successfully received by at least one station having the data packet. Since all the stations are assumed to receive a retransmission request packet independently with probability $1-p_{R}$, and other stations cancel their retransmission requests upon hearing one, the probability we are looking for is

$$
p_{\text {success }}=\sum_{k=1}^{N-i}\left(\begin{array}{c}
N-i \\
k
\end{array}\right) p_{R}^{(N-i-k)}\left(1-p_{R}\right)^{k}=1-p_{R}^{N-i}
$$

If at least one station receives the retransmission request packet correctly the data packet is retransmitted. The system state changes to another state $j, j \leq i$, as a result of this reply, see Fig. 3.5. It implies that $i-j$ stations out of $i$ stations have received the reply packet correctly, while the remaining $j$ stations have not 
received it due to transmission loss. The probability of the above-mentioned event (denoted by $B\left(i, p_{T}, j\right)$ ) is given by

$$
B\left(i, p_{T}, j\right)=\left(\begin{array}{c}
i \\
i-j
\end{array}\right) p_{T}^{j}\left(1-p_{T}\right)^{(i-j)}
$$

Observe that the latter event is conditioned on at least one station having the original data packet successfully receiving the retransmission request packet first. Consequently, we get the probability of the system transition from the state $i$ to the state $j$ as

$$
q_{i j}=\sum_{k=1}^{N-i}\left(\begin{array}{c}
N-i \\
k
\end{array}\right) p_{R}^{(N-i-k)}\left(1-p_{R}\right)^{k}\left(\begin{array}{c}
i \\
i-j
\end{array}\right) p_{T}^{j}\left(1-p_{T}\right)^{(i-j)}
$$

where $i=0,1, \ldots, N$ and $j=0,1, \ldots, i-1$.

Note that there is still a chance that no stations get the data packet correctly at the end of the retransmission attempt. This could happen due to no station getting the retransmission request correctly or no stations getting the data packet correctly. This probability is given by

$$
q_{i i}=p_{R}^{(N-i)}+\sum_{k=1}^{N-i}\left(\begin{array}{c}
N-i \\
k
\end{array}\right) p_{R}^{(N-i-k)}\left(1-p_{R}\right)^{k} p_{T}^{i}
$$

where $i=0,1, \ldots, N$ and it is the probability of remaining in the same state.

Finally, the elements of $\vec{q}$ are obtained observing that the only way to approach the state 0 from any arbitrary transient state $i$ is receiving the data packet correctly by all $i$ stations. The corresponding probability is given by

$$
q_{i 0}=\sum_{k=1}^{N-i}\left(\begin{array}{c}
N-i \\
k
\end{array}\right) p_{R}^{(N-i-k)}\left(1-p_{R}\right)^{k}\left(1-p_{T}\right)^{i}
$$


This completes the parameterization of the model.

\subsection{Performance analysis}

Below, for the proposed model, we derive the performance metrics of interest. These include (i) probability of complete delivery, (ii) $95 \%$ quantile of the number of (re)transmission attempts required to deliver a packet to all the stations, (iii) Probability Mass Function (PMF) of the number of (re)transmission attempts required to deliver a packet to all the stations, (iv) residual loss probability, (v) overhead of error recovery, (vi) mean delay to deliver a packet to all the stations and (vii) mean delay per receiver.

\subsubsection{Evaluation metrics}

Probability of complete delivery The first metric we are interested in is the probability that a packet is delivered to all receivers within $k$ steps. So, for the Markov model defined in Eq. 3.2 we are interested in computing the probability of absorption in no more than $k$ steps. Observe that the time to absorption in a Markov chain with one absorbing state is in fact a First Passage Time (FPT) distribution expressing the number of steps required to get into the absorbed state from the set of all transient states $1,2, \ldots, N$. This metric characterizes the number of (re)transmission attempts that must be performed to deliver a packet to all the stations.

Let $D$ denote the random variable describing the number of steps till absorption. Practically, $D$ describes the number of (re)transmissions that has to be performed to get the packet distributed to all the stations. The Cumulative Distribution Function (CDF) of $D$ is given by [77]

$$
F_{D}(k)=\operatorname{Pr}(D \leq k)=1-\vec{h}^{\prime}\left(Q^{k}\right) \vec{e}
$$

where $\vec{h}^{\prime}$ is the initial state probability vector, without $h_{0}$. 
95\% Quantile Relevant to CDF of $D$, we also consider quantile of the number of (re)transmission attempts as a measure to get insight regarding the trade-off between the probability of absorption and the relevant overhead. We consider $95 \%$ quantile, translated to the number of (re)transmission attempts for which the CDF of $D$ is higher that $95 \%$.

PMF of the number of (re)transmission attempts The PMF of the number of (re)transmission attempts can be derived from CDF by differentiating as

$$
\begin{aligned}
& f_{D}(0)=F_{D}(0), \\
& f_{D}(k)=F_{D}(k)-F_{D}(k-1), k=1,2, \ldots, K,
\end{aligned}
$$

which represents the probability of absorption in exactly $k$ (re)transmission steps.

Residual loss probability As the name implies, this performance metric represents the residual probability of loss for an arbitrary station, given that $K$ (re)transmission steps are allowed. It is computed as

$$
p_{\text {res }}^{K}=\sum_{i=1}^{N} i \cdot P_{i}^{K} / N,
$$

where $P_{i}^{K}$ represents the probability of being in the state $i$ after $k=K$ (re)transmission attempts as defined in equation 3.8.

Overhead of error recovery Analyzing a (re)transmission-based error recovery technique, we have to quantify how much overhead is imposed by (re)transmissions, i.e., the expense of the proposed higher layer reliability protocol. This metric can be defined in terms of the expected number of transmissions and derived by averaging over the absorption probabilities for all the 
given number of (re)transmission steps as

$$
H=1+\sum_{k=1}^{\infty} f_{D}(k) \cdot k .
$$

Mean packet distribution delay Consider now the mean delay performance of the protocol, more specifically, the expected delay until all stations have received the packet. Note that we will explicitly take into account the delay introduced by the back-off procedure. In the derivation below we will need the notion of the fundamental matrix of an absorbing Markov chain that we will introduce next.

According to the definition of the matrix $Q$ in Eq. 3.2, it is inferrable that the probability of transition from the state $i$ to the state $j$ in exactly $k$ steps is $(i, j)^{t h}$ entry of $Q^{k}$. A basic property of an absorbing Markov chain is the fundamental matrix of the chain defined as summation of $Q^{k}$ over all $k$ 's [77] as

$$
Z=\sum_{k=0}^{\infty} Q^{k}=(I-Q)^{-1}
$$

where $I$ is the identity matrix of size $N \times N$. The $(i, j)^{t h}$ entry of the fundamental matrix $Z$ represents the expected number of visits to a transient state $j$ prior to absorption, assuming the system starts at a transient state $i$.

Observe that the sojourn time in a state of the proposed model is a random variable. The mean transmission delay consists of the mean time required to perform the initial transmission followed by the mean time spent for all consecutive requests and replies until all the stations correctly receive the packet. During this time the system traverses from the initial state to the absorbing state by passing through transient states. Therefore, at first we need to know the probability of visiting either of the system states and later the amount of time spent in each of them till absorption, which may vary from state to state. The probability of visiting any arbitrary transient state $j$ of an absorbing Markov chain when starting at a transient state $i$ [77], is given by the $(i, j)^{t h}$ 
element of the following matrix

$$
W=(Z-I)\left(Z_{D}\right)^{-1}
$$

where, $Z_{D}$ is the diagonal matrix derived from the fundamental matrix $Z$ by setting all its elements to zero, except the diagonal elements. Recalling that $q_{i i}$ is the probability of staying in the state $i$, we see that the time in a state of the Markov chain is geometrically distributed with parameter $q_{i i}$. Further, as each requester station sets a constant time out, $T_{\text {timeout }}$, to receive the reply, we get the mean total duration of time it takes to perform (re)transmission attempts given that they fail, denoted by $E\left[T_{i} \mid F\right]$, as

$$
E\left[T_{i} \mid F\right]=\frac{1}{q_{i i}} T_{\text {timeout }}
$$

Let us now look at the time needed for the final, successful (re)transmission. That consists of the back-off time plus packet transmission time for both the (re)transmission request and the (re)transmission itself. We approximate the number of time slots before sending a reply by assuming that all $N-i$ stations that have the packet do receive the request. Recalling that all the stations missing the packet, choose the back-off counter from a geometric distribution with the same parameter $r_{R}$ to send a (re)transmission request, the number of time slots to access the channel is geometrically distributed with parameter

$$
r_{R}^{*}=1-\prod_{l=1}^{i}\left(1-r_{R}\right)=1-\left(1-r_{R}\right)^{i},
$$

which follows from the definition of the minimum of a set of random variables and the memoryless property of a geometric distribution. Similarly, the number of time slots to access the channel for sending the data packet is geometrically distributed with parameter

$$
r_{T}^{*}=1-\prod_{k=1}^{N-i}\left(1-r_{T}\right)=1-\left(1-r_{T}\right)^{N-i} .
$$


Now, recalling that $T_{T}$ and $T_{R}$ are the transmission time of a data packet and a request packet respectively, we could find the average residence time at the arbitrary state $i$, denoted by $E\left[T_{i}\right]$, as

$$
E\left[T_{i}\right]=E\left[T_{i} \mid F\right]+\frac{T_{\text {slot }}}{r_{R}^{*}}+T_{R}+\frac{T_{\text {slot }}}{r_{T}^{*}}+T_{T},
$$

where the first component accounts for the time spent due to $\frac{1}{q_{i i}}$ time failures and the rest considers the time spent to leave the state $i$ successfully. That is, the time prior to access the channel to send a request as $1 / r_{R}^{*}$ times $T_{\text {slot }}$ plus the transmission time of the request packet, $T_{R}$, provided that at least one station possessing the packet receives the request. Then after, it is summed up with the time prior to access the channel to send a reply as $1 / r_{T}^{*}$ times $T_{\text {slot }}$ plus transmission time of the reply packet, $T_{T}$, provided that at least one station missing the packet, receives the reply.

Summing up Eq. 3.23 over all states gives the mean packet distribution delay, i.e.,

$$
E[T]=T_{T}+\sum_{j=1}^{N} \sum_{i=1}^{j} h_{j} \cdot w_{j i} E\left[T_{i}\right],
$$

where $T_{T}$ is the time for the first original transmission, and $w_{j i}$ is the probability of visiting the arbitrary state $i$ starting from the arbitrary state $j$ of the chain, which is represented by $h_{j}$.

Mean delay per receiver Beside the mean delay performance of the system, i.e., the expected time until all stations have received the packet, it is of interest to find out the delay for a given station. That is, for each individual station the expected time until it receives the packet for the first time. This metric, $E\left[T_{S}\right]$, can be derived as

$$
E\left[T_{S}\right]=\sum_{j=1}^{N} \sum_{i=1}^{j} h_{j} \cdot w_{j i} E\left[T_{i}\right] \cdot N-i / N
$$


Table 3.2: Numerical values.

\begin{tabular}{cccccccccc}
\hline \hline Parameter & $K$ & $p_{R}$ & $p_{T}$ & $r_{R}$ & $r_{T}$ & $T_{\text {timeout }}$ & $T_{\text {slot }}$ & $T_{R}$ & $T_{T}$ \\
\hline Value & \multirow{2}{*}{30} & 0 & 0.1 & 0.5 & 0.5 & 0.012 & 0.0001 & 0.001 & 0.01 \\
\hline
\end{tabular}

summing up all the combinations of "ending up at a given state $j$ after the original broadcast, visiting a given state $i$ directly after state $j$ and residing at this state for $E\left[T_{i}\right]$ amount of time". This is basically the delay experienced by $N-i / N$ of receivers. Hence, we weight the delay by this fraction.

\subsubsection{Numerical results}

In this section we first validate the proposed model using simulations and then analyze the performance of the protocol in detail. To validate our model, the system has been implemented in the NS-3 network simulator. Further, various realistic scenarios have been analyzed using a Wolfram Mathematica implementation of the proposed analytical model. The number of nodes, data packet loss probability, the maximum number of allowed (re)transmission requests, packet transmission times and back-off timers are the inputs to the model. Accordingly, we get the delay, loss, delivery and overhead related metrics as the output.

The model has been validated by implementing the proposed protocol in NS-3 environment. That is, following model assumptions, a one-hop wireless network has been set up with the constant position mobility model and negligible propagation loss and delay. Transmission delay and probabilistic packet loss for data packets and (re)transmission request packets have been implemented at the application level to set conditions similar to those of the analytical framework. Also the reliable broadcast mechanism has been implemented at the application level on all nodes. Note that the original broadcast of the data packet is included in the number of transmissions, demonstrated in various results in this section.

Figure 3.6a shows in logarithmic scale the comparison of simulations with the results obtained using the analytical model. The metric of interest is the mean packet distribution delay (See Eq. 3.24) as a function of the number of receivers $N$ in the system. The numerical values used for the experiments are summarized in Table 3.2. The confidence limits were estimated assuming the level of significance $\alpha=0.1$ (two-sided symmetric test, $\alpha / 2=0.05$ ). Though within the confidence limits, the analytical results slightly overestimate the delay 


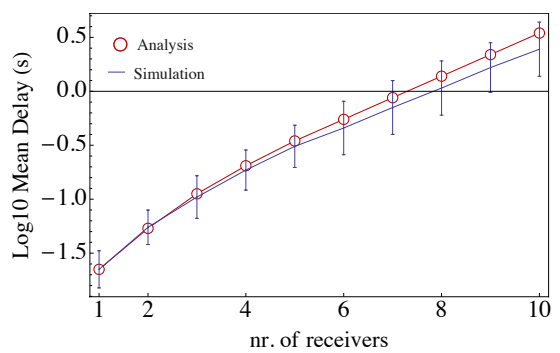

(a) $p_{T}=0.1$ and $p_{R}=0$.

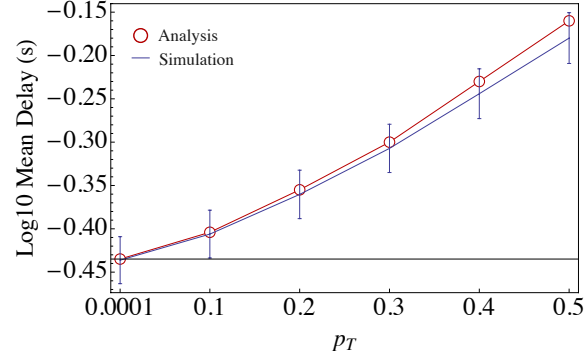

(b) 5 receivers.

Figure 3.6: Mean packet distribution delay.

values due to simplifications of the mathematical modeling. For other values of $p_{T}$ and $p_{R}$ the model follows the simulations closely as well.

Figure $3.6 \mathrm{~b}$ shows the mean packet delay as a function of the data packet loss probability, $p_{T}$, in logarithmic scale for the same set of numerical values (as for Figure 3.6a). The number of receivers was set to $N=5$ and the confidence limits were estimated assuming $\alpha=0.1$. As one may observe the analytical results are within the confidence limits for almost all values of the data packet loss probability, $p_{T}$. The model follows the simulations for other values of $N$ as well. Thus, in what follows, studying the qualitative and quantitative behavior of the system, we will use the developed analytical model only.

Now we present the results obtained from analytical modelling. Note that different numerical settings have been used and the details of each evaluation scenario are described in the corresponding part. For all results to be fitted reasonably, logarithmic scales are used.

\section{Delivery-related metrics}

Figure 3.7a and Figure 3.7b demonstrate the CDF of the number of (re)transmission steps till all the stations get the packet (i.e., probability of complete delivery). That is, the probability of absorption of the proposed Markov model within $K$ number of steps (i.e., in less than or equal to $K$ number of steps) as defined in equation 3.14.

Figure 3.8 demonstrates the $95 \%$ quantile of the number of steps till a broadcast packet is successfully received by all the receivers, as a function of the number of receivers and data packet loss probability. It is important to highlight that even for rather large number of nodes (e.g., $N=10)$ at most three attempts are needed to distribute a packet. Also, observing Figure 3.8 one may notice 


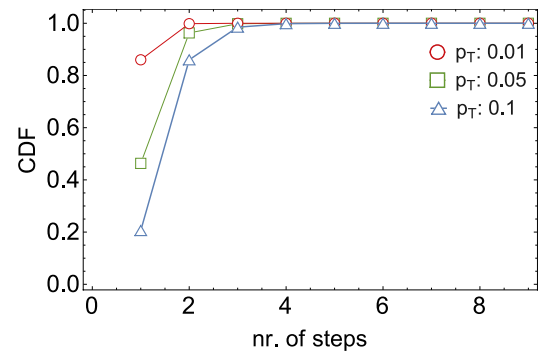

(a) Various $p_{T}, N=15$.

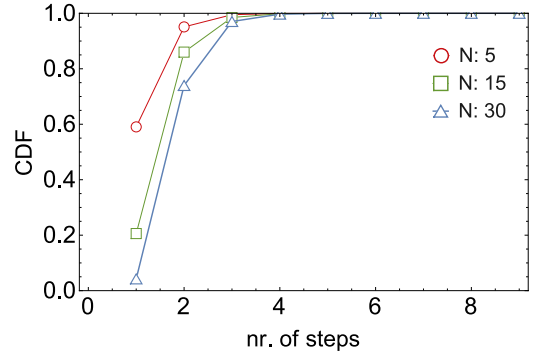

(b) Various $N, p_{T}=0.1$.

Figure 3.7: Probability of complete delivery.

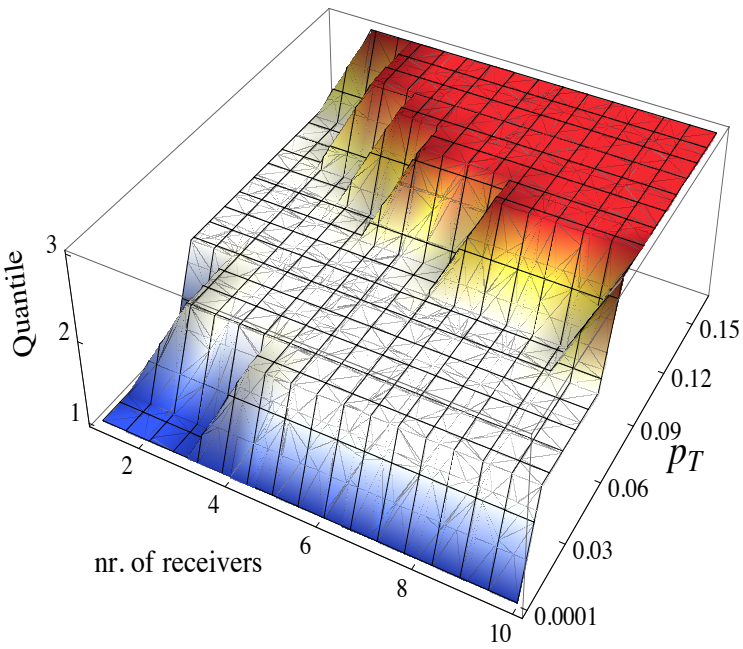

Figure 3.8: 0.95 quantile of the number of (re)transmission attempts.

that the 0.95 quantile of number of steps till a packet is successfully received is not increasing largely with the increase in the data packet loss probability.

Figure 3.9 demonstrates PMFs of the number of steps till a broadcast packet is successfully received by all the receivers (See Eq. 3.15 ) for $N=5,15,30$ and different data packet loss probabilities, $p_{T}$. Note that the probability of incorrect reception of the (re)transmission request packet, $p_{R}$, is set to zero. Recall, that this distribution is a FPT till absorption in our discrete time Markov chain model. As one may observe, for small values of $p_{T}$ the PMF nearly follows geometric distribution. When $p_{T}$ or $N$ or both get higher the distribution is 


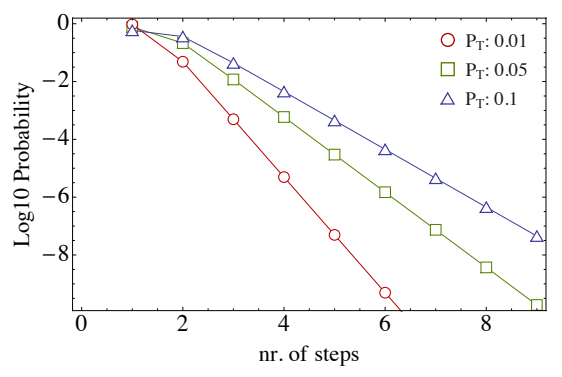

(a) $\mathrm{N}=5$ receivers.

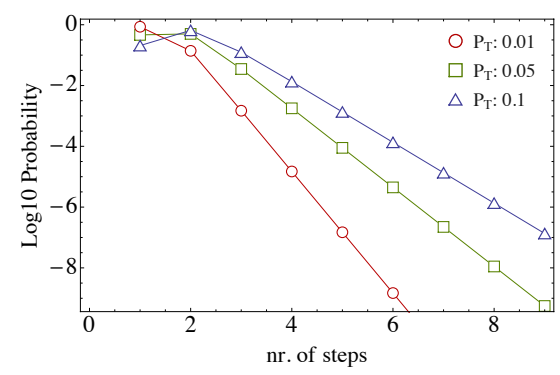

(b) $\mathrm{N}=15$ receivers.

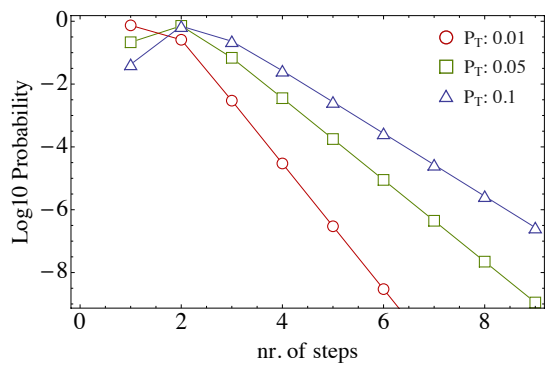

(c) $\mathrm{N}=30$ receivers.

Figure 3.9: PMF of the number of (re)transmission attempts for different number of receivers, $N$.

no longer geometric and resembles the properties of binomial distribution. For all the input parameters the probability of absorption (i.e., the probability of reaching all receivers) exponentially decreases when the number of steps increases implying that the considered system is characterized by a lightweight tail distribution. The latter is of special importance for practical application of the protocol allowing to set the numbers of (re)transmission attempts to reasonable values. As we will observe below this behavior heavily affects other metrics of interest as well.

Figure 3.10 provides the residual loss probability (See Eq. 3.16) for an arbitrary station for different data packet loss probabilities against increasing number of stations. Recall that this metric is the probability that after $k$ (re)transmission attempts a given station still misses the broadcast data. As one may observe it is extremely small even for large data packet loss probabilities implying that few (re)transmission attempts are sufficient to cover most of the stations. In Figure 3.10a and Figure $3.10 \mathrm{~b}, p_{R}=0$. The probability stays relatively flat as the number of stations increases. The practical consequences 


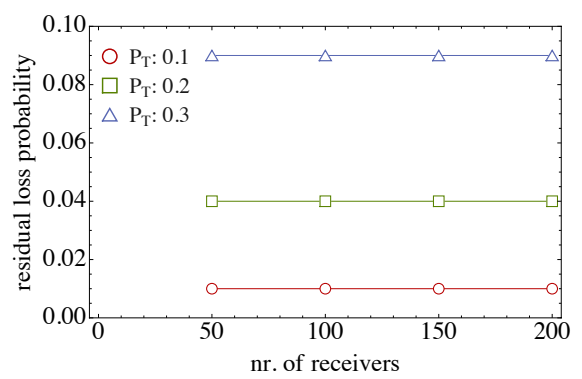

(a) After $\mathrm{k}=2$ (re)transmission trials, $p_{R}=0$.

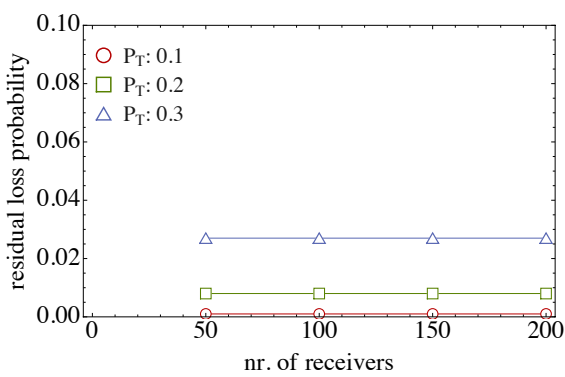

(b) After $\mathrm{k}=3$ (re)transmission trials, $p_{R}=0$.

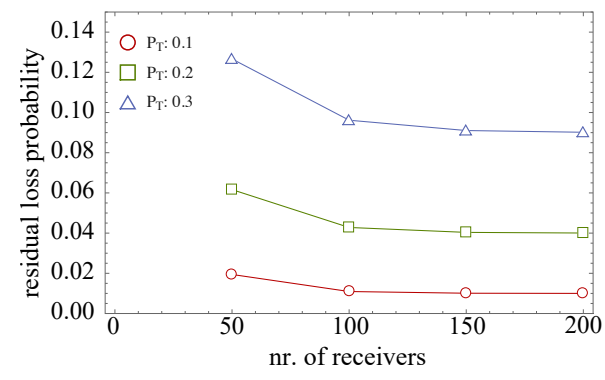

(c) After $\mathrm{k}=2$ (re)transmission trials, $p_{R}=0.5$.

Figure 3.10: Residual loss probability.

are important as it implies that the outcome of the packet distribution is rather independent from the value of $N$ in large distribution scenarios, while $p_{R}=0$. By having higher values for $p_{R}$, we expect that by increasing the number of stations, the chance of getting the packet increases slightly, as it is confirmed by Figure $3.10 \mathrm{c}$ where $p_{R}=0.5$, because other stations transmitting a (re)transmission request could compensate for a station's (re)transmission request being lost.

Another metric of interest we address is the probability distribution of the number of unsuccessful receivers after $k$ (re)transmission attempts. It is the $i^{\text {th }}$ element of the state probability vector given by multiplying the initial state probability vector to the transition probability matrix to the power of $k$, as given by Eq. 3.8. Figure 3.11 illustrates this probability distribution for one and two (re)transmission attempts for $N=50$. Observing it one may deduce that increasing the packet loss probability the probability of not being absorbed in the states with higher numbers increases, while the curve gets wider as well, implying more relaxed probability distribution over the range of system states, 


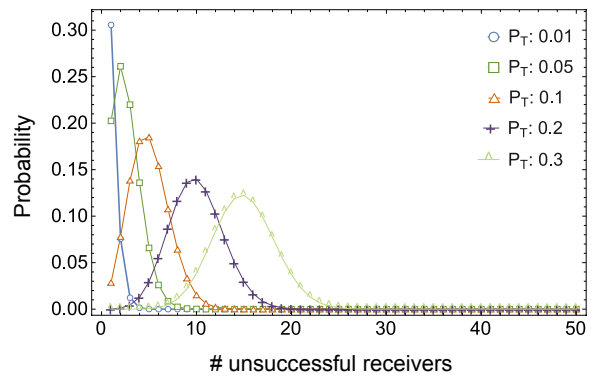

(a) Various $p_{T}, \mathrm{k}=1$.

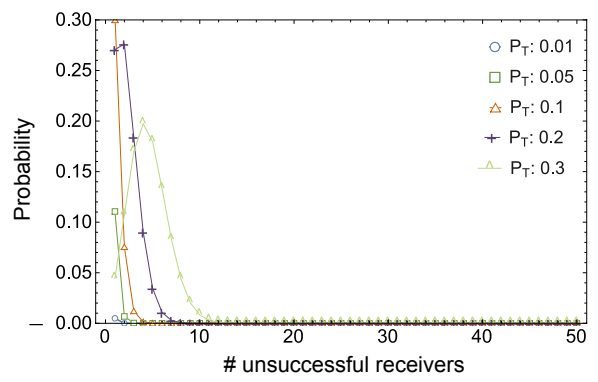

(b) Various $p_{T}, \mathrm{k}=2$.

Figure 3.11: Probability distribution of the number of unsuccessful receivers after $k$ attempts.

which is exactly 50 for this case. The evolution of the system states can be observed comparing PMFs after one and two (re)transmission requests. As we see the PMF for two (re)transmission attempts has its probability mass concentrated closer to zero implying that the number of stations not having received the packet decreases.

Figure 3.12 shows the number of (re)transmissions representing the overhead of error recovery versus the data packet loss probability and number of receivers. As expected, the number of (re)transmissions required for successful delivery of the packet increases either by increasing the data packet loss probability and/or number of receivers. For smaller values of loss probability the required number of (re)transmissions does not change noticeably when the number of receivers increases. However, for higher values of loss probability there is bigger gap among the number of (re)transmissions needed for different number of receivers. Note that the first step presents the original transmission of the packet.

\section{Delay-related metrics}

Figure 3.13a and Figure 3.13b demonstrate the mean delay experienced by an arbitrary station to receive the packet (See Eq. 3.25) for different number of receivers and packet loss probability $p_{T}$, respectively. As one may observe the delay increases exponentially when $p_{T}$ increases. While this is not a desirable feature as we always prefer a linear increase, such exponential increase is inherent for most communication protocols. Results, similar to those illustrated in Figure 3.13a and Figure 3.13b could be used to assess protocol's delay behavior quantitatively for any set of input parameters. 


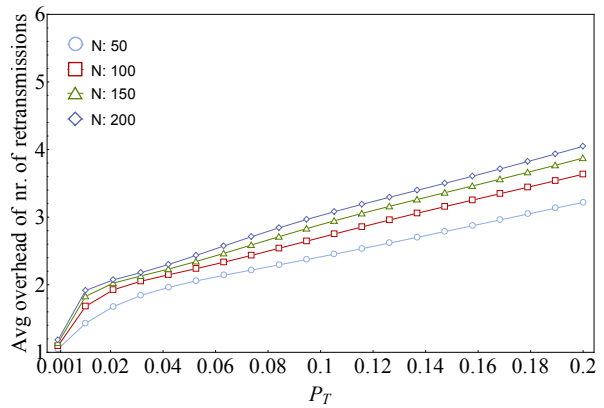

(a) Various nr. of receivers.

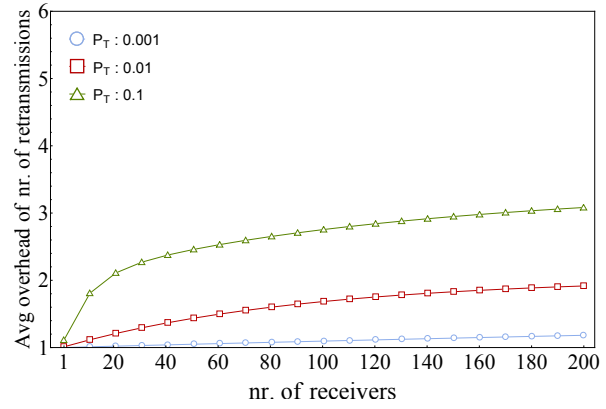

(b) Various $p_{T}$.

Figure 3.12: Overhead of error recovery in terms of the nr. of (re)transmissions.

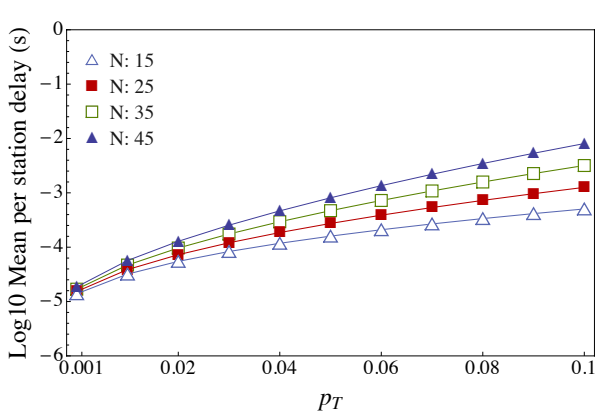

(a) Various $N$.

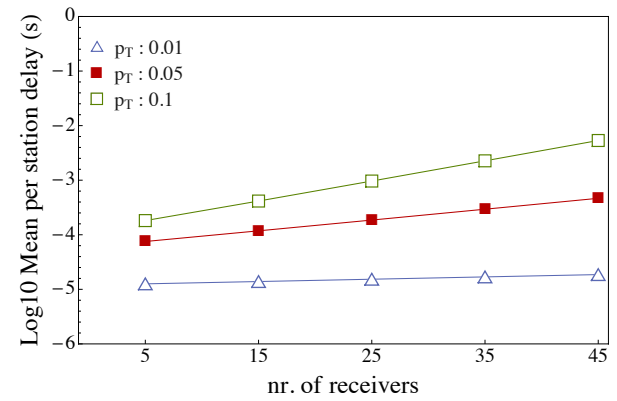

(b) Various $p_{T}$.

Figure 3.13: Mean per receiver packet distribution delay.

Figure 3.14 quantifies the effect of input parameters on the mean delay response of the system (See Eq. 3.24). In particular, the mean delay as a function of the number of receivers for three different data packet loss probabilities is shown in Figure 3.14a. As expected, the mean packet delay increases nearly exponentially as the number of receiving nodes $N$ increases. The effect of the data packet loss probability is also exponential for small values of $p_{T}$ as shown in logarithmic scale in Figure 3.14b. However, for larger values of $p_{T}$ the mean delay increases linearly rather than exponentially for all considered values of the number of receiving stations. It reveals that the number of nodes failing to successfully receive the packet increases as either the number of nodes or the data packet loss probability increases. Accordingly, higher number of nodes back-off to send (re)transmission requests. For all the missing nodes to receive 


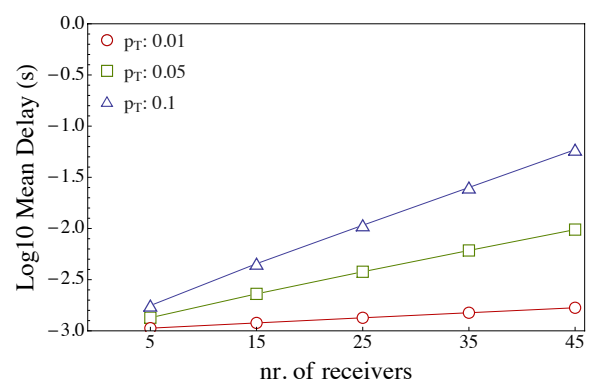

(a) Different $p_{T}$.

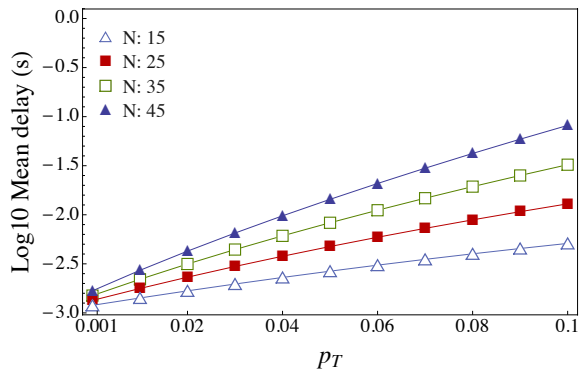

(b) Different $N$.

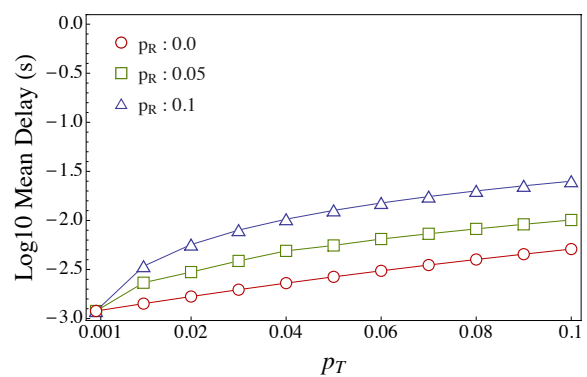

(c) Different $p_{R}$.

Figure 3.14: Mean packet distribution delay.

the packet, higher number of (re)transmission requests are sent, resulting in the increased mean E2E delay. Note that in Figure 3.14a and Figure 3.14b the (re)transmission request packet loss probability is set to zero.

Finally, Figure 3.14c demonstrates the mean delay as a function of the data packet loss probability for three different (re)transmission request loss probabilities and $N=15$. As one may observe, the effect is similar to that of the data packet loss probability $p_{T}$. Additionally, when $p_{T}$ and $p_{R}$ are both not zero the effect is qualitatively similar.

\subsection{Concluding remarks}

In this chapter we proposed and E2E reliability assurance mechanism for vehicular (geo-)broadcast. We developed an analytical approach of modeling the proposed mechanism and we evaluated its performance, in terms of relevant metrics, via experiments based on the analytical model. We demonstrated that it captures the protocol behavior reasonably by validating the model via NS-3 
simulations. The specifications, playing the main role in system performance, were considered as inputs to the model. Accordingly, we obtained and analyzed system-oriented performance measures and an extended set of results, providing a deeper insight to the system. The proposed model is general enough and allows for a number of extensions.

In relevance to time-critical vehicular safety applications, our main performance metric is E2E delay and the model demonstrates that the delay performance of the system scales exponentially with the increase in the number of stations and (data and retransmission request packet) loss probabilities. Hence, while sender-based reliability approaches do have scalability problems due to storms of acknowledgements, this receiver-based approach is also limited in its scalability if full reliability is to be achieved. Nevetheless, it is apparent from our analysis that even with high number of receivers and high packet loss probabilities, data can be reliably distributed within a limited number of retransmission attempts. The analysis shows that in a single-hop scenario, receiver-based reliable broadcasting can provide good reliability, while giving very little overhead for high number of receivers.

The current model contains several assumptions, mainly it takes the effect of collisions into account only through a load-independent loss probability. For systems with low number of nodes (up until approximately 20) and/or large contention windows the proposed system demonstrates results close to simulations. However, when these requirements are not satisfied we have to explicitly take collisions into account. The current model applies for the one-hop communication scenario and modeling the protocol behaviour in a multi-hop communication scenario is the focus of the next chapter. 



\section{Reliable V2V broadcast: multi-hop communication scenario}

In vehicular networks, nodes may act as relays for further dissemination of information. In this chapter, we aim to evaluate performance of the reliability mechanism from Chapter 3 in the context of multi-hop broadcast. First, we analytically model multi-hop broadcasting and subsequently the reliability mechanism. Using absorbing Markov modeling and probabilistic graphical modeling, we analyze its performance in terms of indicators, such as overhead and delivery ratio. The model is also validated using simulations. The results are useful in tuning the influential parameters to meet performance requirements in various circumstances. The model is not limited to a specific network topology. Hence, network characteristics such as the number of one-hop neighbors, affecting dissemination penetration rate, are considered. Via experiments, confirming the impact of the topology and the connectivity regime on the broadcast performance, we provide insight into the system functionality. The publications related to this chapter are [64, 63].

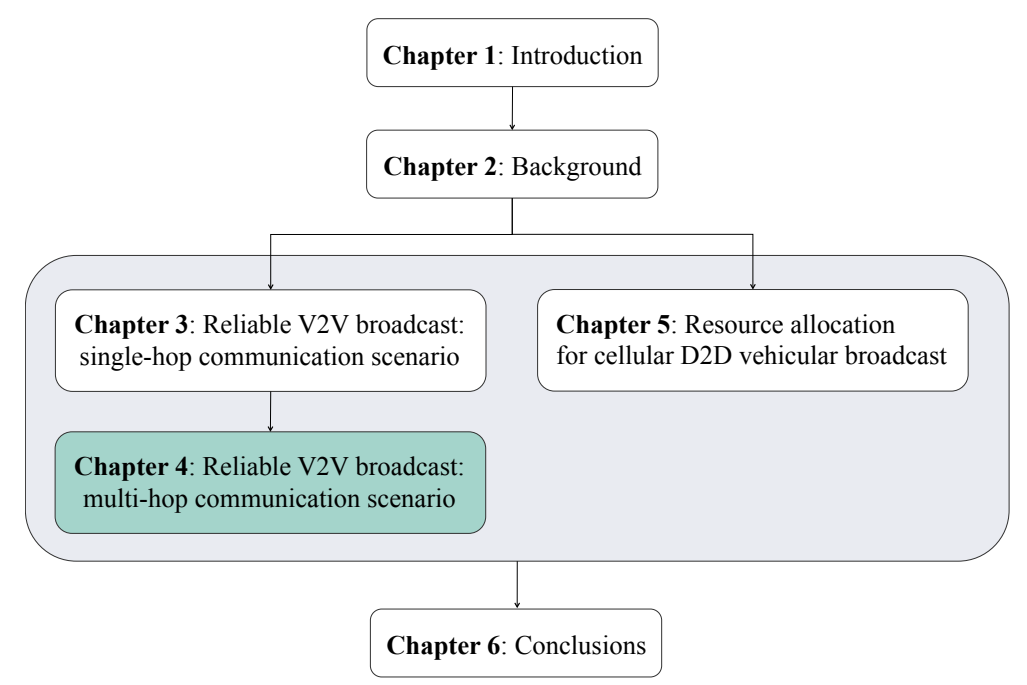


This chapter is organized as follows:

- Section 4.1 gives an introduction to vehicular multi-hop broadcasting and states the contribution of this chapter.

- Section 4.2 starts with discussing some work of relevance. The functionality of the proposed multi-hop broadcast approach and its modeling are introduced in Section 4.2.2. This includes the considered scenario and parameterization in Section 4.2.2.1 and Section 4.2.2.2, respectively. Subsequently, Section 4.2.3 is devoted to performance analysis of the proposed multi-hop broadcast approach, in terms of the relevant metrics.

- Section 4.3 focuses on the application of the reliability mechanism, introduced in Section 3.3, to the multi-hop broadcast scenario, introduced in Section 4.2.2. It starts with Section 4.3.1 discussing the literature regarding reliability improvement of multi-hop broadcast in vehicular networks. Section 4.3.3 introduces the approach of modeling the integrated multi-hop broadcast and the reliability mechanism, preceded by a brief reminder of the proposed reliability mechanism in Section 4.3.2. Section 4.3.4 is devoted to first introducing the performance indicators that we use to evaluate the system and accordingly presenting the results of validating the analytical model, using simulations. It continues by presenting extensive numerical results of performance evaluation, in terms of specified indicators.

- Section 4.4 concludes this chapter. 


\subsection{Introduction}

In Chapter 3, we analytically modeled and evaluated a receiver-based E2E reliability assurance mechanism for vehicular networks in the context of singlehop (geo-)broadcast. This mechanism enables loss detection and correction by means of sequence numbering and checking at communicating parties, as shown at high-level in Figure 4.1 and is suitable for various ITS applications, demanding strict requirements on delivery assurance. In this chapter we aim to model the aforementioned reliability mechanism in the context of multi-hop broadcast. That is, those nodes failing to receive a packet after several hops of rebroadcasts and having detected such a loss through this mechanism, will broadcast retransmission requests to their one-hop neighborhood. Accordingly, the neighbors possessing that missing packet will reply by retransmitting it. For this, at first we need to model the behavior of information dissemination by means of multi-hop broadcast. Next, by taking the results into account, we continue by analyzing the performance of the aforementioned on-demand error recovery mechanism.

Whereas simulations and experimental results can provide accurate performance figures for specific parameter settings, analytical modeling, as provided in this chapter, can give more fundamental insights and quick results for many different parameter settings. Our analytical approach is not based on a specific network scenario and can provide results for various settings and accordingly basic insights regarding the system behavior. As a result of this, our model is extensible by introducing more detail into the parameterization of the model.

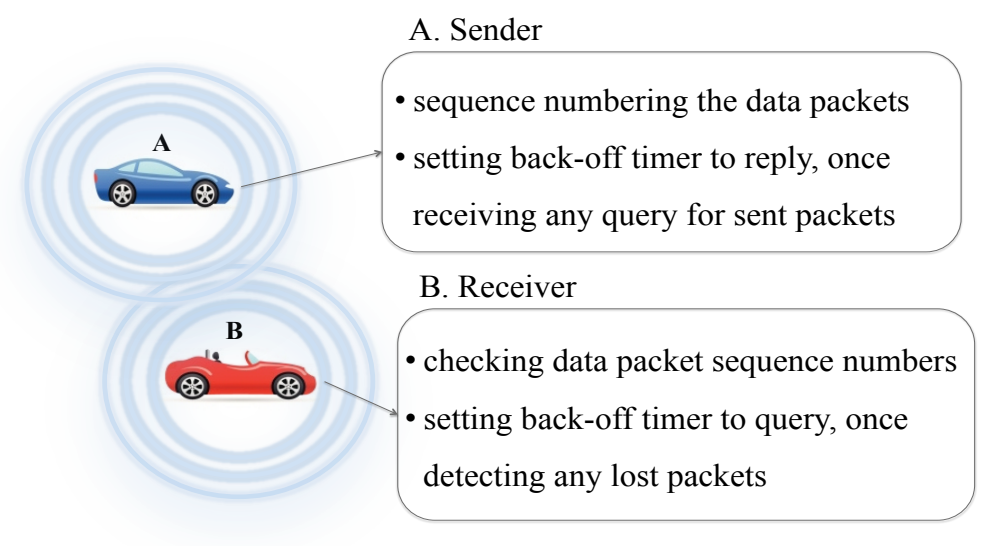

Figure 4.1: The operation principle of the E2E reliablility mechanism. 
The main contributions of this chapter are as follows:

- we derive a mathematical model of probabilistic multi-hop broadcast;

- we analyze its performance using the analytical model, in terms of the probability of receiving a broadcast packet;

- we provide fundamental insight into the behavior of multi-hop broadcast;

- we analytically model the error recovery process in the context of multihop broadcasting;

- based on the analytical model, we analyze performance of the error recovery phase;

- we validate observations from the analytical analysis, using simulations.

\subsection{Multi-hop broadcast}

\subsubsection{Related work}

V2V broadcast communication and accordingly multi-hop connectivity as an evolving research area has been approached by the research community from various perspectives, analytically or via simulations. For instance, some researchers may consider measures such as critical transmission range or vehicle density for network connectivity, while others may also consider delivery ratio, covered neighborhood, redundant traffic overhead or propagation delay. In this section, we point some of the most relevant ones regarding performance analysis.

The work in [71] proposed a Markov process to analytically model onedimensional multi-hop broadcasting. The received signal power at a node is used to derive the state transition probabilities of the proposed Markov chain. The authors in [56] based their analysis on transmission range and rate as metrics affecting the performance of hidden node affected multi-hop broadcasting in a one-dimensional topology. Khabazian et al. proposed in [78] the analytical performance modeling of multi-hop broadcast by differentiating between two priority classes. Using a two-dimensional Markov model, the authors derived the distribution of the number of low priority messages, in both transmission and back-off modes. Accordingly, they came up with the average dissemination delay of high priority messages, considering low priority messages present in the background. In [61], Fracchia et al. analytically modeled inter-vehicular multi-hop broadcast with respect to deriving the probability distribution of the number of informed vehicles and the average delay as the main performance 
measures. The authors in [61] based dissemination reliability on network connectivity. They proposed an analytical model to predict the number of nodes in the neighbourhood and accordingly adjust the probability of rebroadcast to achieve better coverage.

Hassanabadi et al. proposed the Synchronized Persistent Coded Repetition (SPCR) algorithm [72] as the main functionality of the reliability sub-layer in the application layer of the WAVE stack to increase the reliability of safety applications; that is, the rebroadcast of network coded messages. They evaluated their approach in terms of the probability of successful reception of messages by a node and also system throughput as the average number of successful messages received during a sync interval. The approach in [147] is based on the volunteers' dilemma game to conduct probabilistic rebroadcast and suppress broadcast storms. The authors evaluated the proposed protocol in terms of forwarding probability, packet delivery ratio and overhead of duplicate packets, via simulations in the NS-3. In [81], Klein Wolterink et al. analytically modeled the performance of piggybacking data on beacons in vehicular networks. Given several relevant input parameters, they captured the performance mainly in terms of the probability distribution of the E2E and per-hop delay. Another work by the same authors [151] is dedicated to analytical modeling of geographical multi-hop broadcast in VANETs as a means of evaluating its performance. Besides the measures discussed in their previous work, they investigated the required number of hops to cover a given one-dimensional dissemination distance. A work by Van Mieghem et al. in [137] analytically examines the influence of network characteristics (in terms of connectivity) on the success rate of virus spread through networks.

The Enhanced Multi-Hop Vehicular Broadcast (MHVB) [97] approach is flooding-based and uses an algorithm to select the right node to rebroadcast and thereby increase reliability. The authors followed a simulation-based evaluation criteria, defining the success rate as the main measure, which is the ratio of the number of packets received within a limited time by a node to the total number of packets received by the same node during the entire simulation time. In [100], relying on simulated scenarios, the authors compared three multi-hop broadcast algorithms as simple flooding, direction-aware flooding, and multipoint relaying. Simple flooding was found to outperform the other two in terms of delay and delivery ratio. In [? ] an E2E geocast acknowledgement scheme is proposed, in which individual ACKs are accumulated into larger messages in an aggregator and further forwarded back over multiple hops to the original source. The authors based their studies on simulations and examined the approach in terms of overhead and reliability.

Whereas most of the related work focuses on simulation studies or analytical analysis, given a particular one-dimensional scenario, in this chapter we develop 
a novel approach of modeling and analyzing the performance of probabilistic multi-hop broadcast, mainly in terms of dissemination reliability. That is, we derive a general analytical model for two-dimensional multi-hop broadcast, incorporating network topology in its basis. Hence, determinant factors of dissemination performance such as network density and the number of 1-hop neighbors are already considered in the model. To the best of our knowledge, this is the first work to quantify the probability distribution for each specific node of a network to have a broadcast packet after a given number of (re)broadcasts. Our model is not based on a particular network setting and is general enough to be applied to a wide variety of scenarios.

\subsubsection{Modeling approach}

This section presents the analytical modeling of multi-hop broadcasting as it proceeds. That is, we analytically model a single cycle of multi-hop broadcast in two-dimensional vehicular networks. The model incorporates network topology in its basis. As a result, the effect of major network characteristics such as network density and the number of 1-hop neighbors are considered in the model as described later in this section. These characteristics play a crucial role in the overall data dissemination. First, we start by describing the considered scenario and afterwards continue by introducing the modeling approach. Further, using the model, we analyze its behavior in terms of probability of possessing the packet being broadcast as an influential performance measure. The notation used throughout this chapter is summarized in the Table 4.1.

Table 4.1: Notation used throughout this chapter.

\begin{tabular}{|c|l|}
\hline Parameter & Definition \\
\hline \hline $\mathrm{N}$ & The number of network nodes (including the source node) \\
\hline $\mathrm{b}$ & The number of the rebroadcasts \\
\hline$p_{T}$ & The probability of successful packet transmission between any pair of reachable nodes \\
\hline$p_{r b}$ & The probability of data packet rebroadcast \\
\hline$R$ & The transmission range $(\mathrm{m})$ \\
\hline $\mathrm{k}$ & The number of the retransmission requests \\
\hline $\mathrm{S}$ & The set of all nodes of a network \\
\hline
\end{tabular}




\subsubsection{Considered scenario}

As shown in an example scenario in Figure 4.2, we consider a certain number of vehicles in a given two-dimensional area (e.g., a 4-lane highway), with a vehicle initiating the broadcast of a packet as the source (labeled as "s" in Figure 4.2) and the rest as the intended receivers of the broadcast data. Nodes labeled as "r" in the figure, act as relays and rebroadcast the received data. As a result of this, data is broadcast and going forward over multi hops beyond the neighborhood of the original source. Various relay node selection strategies have been proposed in the literature and in this work we consider probabilistic relaying [39] (with probability $p_{r b}$ ) by the receiver nodes. It is assumed that just vehicles within

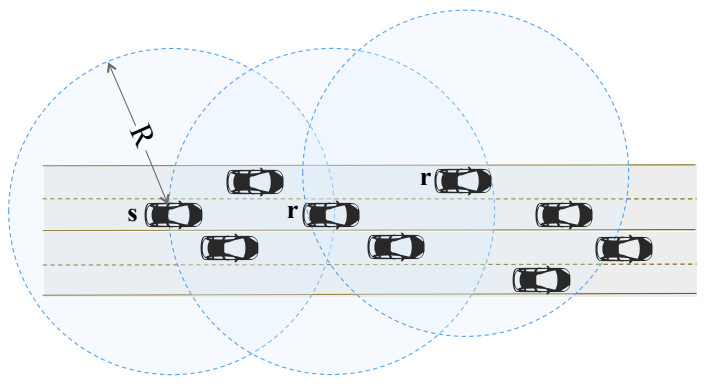

Figure 4.2: Broadcast over multi-hops beyond the neighborhood.

a given threshold distance (i.e., transmission range $R$ ) can hear each other, according to a connectivity function of effective factors (e.g., transmission rate, propagation loss, hidden nodes and collision). One-hop neighbors of the source, who successfully receive the packet for the first time, will start a back-off timer to rebroadcast the packet as a means to avoid potential collisions due to concurrent rebroadcasts. Further, upon expiration of the timer, these neighbors will forward the packet with a certain probability $p_{r b}$. The packet is rebroadcast in a multihop manner to cover the destination neighborhood.

\subsubsection{Modelling and parameterization}

Given the described scenario, we model the (re)broadcast flow as follows. Let tuple $T=\left(N, R, U\left(\left(x_{\min }, x_{\max }\right),\left(y_{\min }, y_{\max }\right)\right)\right) \quad$ denote a network topology of $N$ nodes with transmission range $R$, uniformly distributed in a two dimensional area $\left(x_{\min }, x_{\max }\right),\left(y_{\min }, y_{\max }\right)$, which is found suitable for the case [148]. Knowing the relative distance between any arbitrary pair of nodes, we can shape a reachability graph, such that any two stations falling out of the threshold dis- 
tance, $R$, are not reachable; otherwise, they are. Accordingly, we construct the $N \times N$ reachability matrix $D$, with elements $d_{i j}$ as

$$
d_{i j}= \begin{cases}1, & \text { if } \sqrt{\left(x_{j}-x_{i}\right)^{2}+\left(y_{j}-y_{i}\right)^{2}} \leq R \\ 0, & \text { otherwise. }\end{cases}
$$

All the diagonal elements are set to 1.

Figure 4.3 shows an example reachability graph of $N=7$ nodes in a $2 \mathrm{~km} \times 2 \mathrm{~km}$ area, with the transmission range $R=300 \mathrm{~m}$ and its corresponding matrix $D$ of reachability.

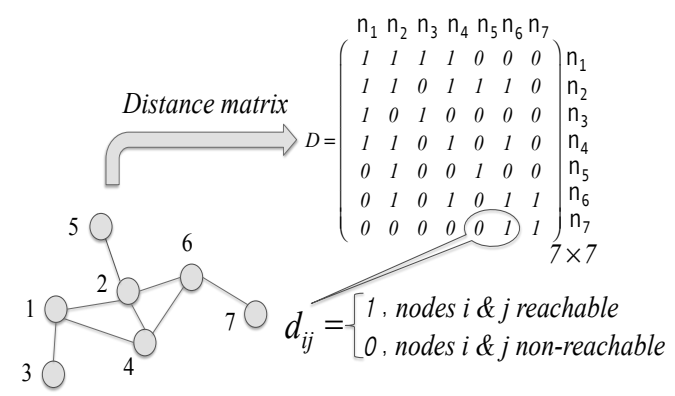

Figure 4.3: The reachability graph of a topology of $N=7$ nodes.

As mentioned earlier in the scenario description, being reachable for two nodes does not necessarily mean that packet transmissions between them are always successful. For this reason, we define the multi-variant function $f\left(\theta_{i j}\right)$, representing the impact of major factors on successful packet transmission between an arbitrary pair of nodes $i$ and $j$, where $\theta_{i j}$ is representative for the set of factors, playing a role in a transmission from node $i$ to $j$. For example, the functions proposed in [56] can be used in integration with our approach. Given this, we define the transmission probability matrix $Q_{T}$, with $i j^{\text {th }}$ element $\left(q_{i j}\right)$, derived by multiplying the corresponding function $f\left(\theta_{i j}\right)$ to the $i j^{t h}$ element of the reachability matrix $D\left(d_{i j}\right)$ (except the diagonal elements) as

$$
q_{i j}= \begin{cases}1, & \text { if } i=j \\ f\left(\theta_{i j}\right), & \text { if } \sqrt{\left(x_{j}-x_{i}\right)^{2}+\left(y_{j}-y_{i}\right)^{2}} \leq R \\ 0, & \text { otherwise. }\end{cases}
$$


Now, given the described scenario, with the source node initiating the broadcast of a packet, we define the following performance measure.

Definition 1. (Packet Delivery Ratio (PDR)). We define the packet delivery ratio as the probability that an arbitrary node, not being the source node, would have a packet, after a given number of (re)broadcasts, $b=0,1, \ldots, B$.

In what follows we are interested to quantify that measure and we begin by framing it as follows:

$$
P D R=\sum_{x \neq s} \frac{P(x, b, S \backslash\{x\})}{N-1},
$$

where $P(x, b, Y)$ denotes the probability for a particular node $x$ to have the packet received from either of nodes in the set $Y$, after $b$ number of rebroadcast steps. $S$ in $P(x, b, S \backslash\{x\})$ denotes the set of all network nodes.

Given this, we aim to find the corresponding general term, $P(x, b, Y)$. As a matter of fact, node $x$ maybe successful in receiving the packet at the original broadcast $(b=0)$ by the source node $s$ or at any of the subsequent rebroadcasts by either of the relaying nodes in set $Y$ (assuming they already received the packet at previous (re)broadcasts) until the $b^{\text {th }}$ step. Given this, we formulate such probability, using the concept of recursive function definition as follows:

$$
P(x, b, Y)= \begin{cases}P(x, b-1, Y)+\left(P_{S, Y}\right)_{x}^{b}, & \text { if } b \geq 1 \\ q_{s x}, & \text { if } b=0 \\ 1, & \text { if } x=s\end{cases}
$$

where the expression in the first line corresponds to the case $b \geq 1$ and it implies node $x$ either has the packet within $b-1$ rebroadcast steps (i.e., the first term

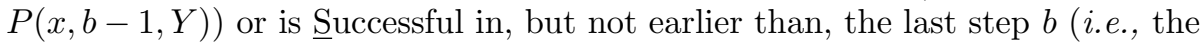
second term represented as $\left.\left(P_{S, Y}\right)_{x}^{b}\right)$. Note that $S$ in $\left(P_{S, Y}\right)_{x}^{b}$ stands for success, not to be confused with the set of nodes $S$ in $P(x, b, S \backslash\{x\})$. The expression in the second line corresponds to the case $b=0$, implying the probability for node $x$ to successfully receive the packet at the original broadcast by the source node which is simply $q_{s x}$ ( i.e., the $s x^{t h}$ element of the transmission probability matrix $Q_{T}$ ). Clearly, if $x=s$, the probability of having the packet is 1 (i.e., the expression in the third line).

Now, we need to find an expression for $\left(P_{S, Y}\right)_{x}^{b}$ in Eq. 4.4. Clearly, the probability for node $x$ to be successful at an arbitrary step makes sense if the 
packet has not been received at all the steps earlier than this step. Hence, we interpret node $x$ to be "successful" at $b^{t h}$ step, if it has Failed to receive the packet until this step (the corresponding probability is denoted by $\left(P_{F, Y}\right)_{x}^{b-1}$ ), but succeeds in Getting the packet exactly at this step from either of the nodes in set $Y$ (the corresponding probability denoted by $\left.\left(P_{G, Y}\right)_{x}^{b}\right)$. Accordingly, $\left(P_{S, Y}\right)_{x}^{b}$ could be decomposed as:

$$
\left(P_{S, Y}\right)_{x}^{b}=\left(P_{F, Y}\right)_{x}^{b-1} \times\left(P_{G, Y}\right)_{x}^{b}
$$

We can formulate $\left(P_{F, Y}\right)_{x}^{b-1}$ in terms of the above-mentioned function as:

$$
\left(P_{F, Y}\right)_{x}^{b-1}=1-P(x, b-1, Y)
$$

As for the second term $\left(P_{G, Y}\right)_{x}^{b}$, the probability of node $x$ receiving the packet exactly at the $b^{\text {th }}$ step, at first it is necessary that at least one node $n$ out of the set $Y \backslash\{x\}$ being successful exactly at $(b-1)^{t h}$ step, and later a successful delivery from node $n$ to node $x$ must take place at $b^{t h}$ step. One may note that here we consider "the probability of node $n$ being successful at exactly the $(b-1)^{t h}$ rebroadcast" and not "the probability of node $n$ having the packet after $(b-1)$ rebroadcasts". The reason is that in the scenario, each node is supposed to rebroadcast the packet just once and it happens once that node gets the packet for the first time. Hence, we differentiate between the mentioned two events to avoid overstating the probability of node $x$ receiving the packet from node $n$ at every single step of rebroadcast until the $(b-1)^{t h}$ rebroadcast. Given this fact, $P(n, b-1, Y \backslash\{x\})-P(n, b-2, Y \backslash\{x\})$ expresses such a probability in terms of the above-defined function. Note that here the set $Y$ excludes node $x$. As mentioned earlier, in order to achieve the condition in which node $x$ gets the packet from node $n$ at step $b$, a successful delivery from node $n$ to node $x$ must take place at the $b^{\text {th }}$ step. Recalling from the already defined transmission probability matrix $Q_{T}$, the probability of successful delivery from $n$ to $x$ at $b^{\text {th }}$ step is derived as $q_{n x}$, assuming initiation of transmission by node $n$ with probability $p_{r b}$, at first. Consequently, we compute $\left(P_{G, Y}\right)_{x}^{b}$ in Eq. 4.5 
as follows:

$$
\left(P_{G, Y}\right)_{x}^{b}=\left\{\begin{array}{l}
1-\prod_{\forall n \in Y \backslash\{x\}}(1- \\
\left(P_{1}(n, b-1, Y \backslash\{x\})-P_{1}(n, b-2, Y \backslash\{x\})\right) \\
\left.\times p_{r b} \times q_{n x}\right), \quad \text { if } b \geq 2 \\
1-\prod_{\forall n \in Y \backslash\{x\}} \\
\left(1-P(n, 0, Y \backslash\{x\}) \times p_{r b} \times q_{n x}\right), \quad \text { if } b=1 \\
q_{s x}, \quad \text { if } b=0 .
\end{array}\right.
$$

One may note from this equation that as $n$ is representative for all network nodes (except $x$ ), we need to take them all into account, since $x$ can receive the packet from either of them. We use the concept of complementary event probability for this purpose. That is, the probability of $x$ getting the packet from either of $n$ nodes is equal to " 1 - the probability of not getting the packet from any of these nodes". Again complementarily, we can calculate the probability of not receiving the packet from node $n$ as " 1 - probability of successfully getting the packet from node $n$ ". Now, we can substitute Eq. 4.7 and Eq. 4.6 in Eq. 4.5. 
Further, by substituting Eq. 4.5 in Eq. 4.4, we arrive at the following expression for $P(x, b, Y)$ :

$$
P(x, b, Y)=\left\{\begin{array}{l}
P(x, b-1, Y)+(1-P(x, b-1, Y)) \times \\
\left(1-\prod_{\forall n \in Y \backslash\{x\}}(1-(P(n, b-1, Y \backslash\{x\})-\right. \\
P(n, b-2, Y \backslash\{x\})) \\
\left.\left.\times p_{r b} \times q_{n x}\right)\right), \quad \text { if } b \geq 2 \\
q_{s x}+\left(1-q_{s x}\right) \times\left(1-\prod_{\forall n \in Y \backslash\{x\}}\right. \\
\left.\left(1-P(n, 0, Y \backslash\{x\}) \times p_{r b} \times q_{n x}\right)\right), \quad \text { if } b=1 \\
q_{s x}, \quad \text { if } b=0,
\end{array}\right.
$$

and herewith, we complete the expression for such a probability metric of the model.

\subsubsection{Performance analysis}

\subsubsection{Preliminaries}

In this section we present results of evaluating the proposed model. We considered influential parameters such as network density, nodes' transmission range, number of rebroadcast steps and packet transmission loss probability as inputs, in order to track their impact on the defined probability metric. Scenarios with various settings have been examined by implementing the described model, partly in Matlab (to generate topologies and corresponding reachability graphs) and Wolfram Mathematica (to obtain the output probability metric) to evaluate the performance of the system in terms of the obtained reliability metric. That is, the average probability of packet reception by an arbitrary node in a network with certain numerical specifications. We consider a two dimensional area of size $2 \mathrm{~km} \times 2 \mathrm{~km}$. In this section, the most remarkable results are highlighted. 
Note that in our implementation, we made some assumptions, such that we consider probability $f\left(\theta_{i j}\right)=p_{T}$ (the probability of successful transmission between any arbitrary pair of in-range nodes) as the indicator of the average effect of dissemination impairment factors, as discussed earlier (e.g., mobility-deriven loss, propagation loss, collisions, hidden nodes, and transmission loss). As a matter of fact, vehicles can be considered in constant positions while data is being disseminated. Hence, we reasonably considered a constant position mobility model. Further, propagation loss is considered to be included in $p_{T}$. However, in more complex scenarios, dissemination functions, dependent to propagation loss, could be embedded into our model, as described earlier in Section 4.2.2. Collisions are considered rather unlikely to happen, due to random back-off timers prior to rebroadcasts. Yet, the effect of collision is considered loadindependently in loss probability $p_{T}$. Considering the scenario, shown in Figure 4.2 , one may note that data is penetrated gradually in the network and in such a setting it is rather unlikely losses due to hidden nodes occur for first-hand receivers of data, in the right direction of data propagation. The rebroadcasting probability $p_{r b}$ is considered 1 and accordingly the number of rebroadcasts $\mathrm{b}$ is considered equal to the number of nodes $\mathrm{N}$, assuming each node rebroadcasts only once, unless it is explicitly mentioned.

Recalling from Eq. 4.8, given a particular scenario, the model captures the probability for each individual node having a broadcast packet after a certain number of rebroadcasts. However, to obtain a more general insight into the behaviour of multi-hop broadcasting, we provide mean results here by taking an average over twenty experimental runs with various topologies for the same numerical setting and $0.95 \%$ confidence intervals. Note that due to overlap of the confidence intervals for different input values, they are presented for limited number of graphs, as a means of better visualization.

\subsubsection{Numerical results}

Figures $4.4 \mathrm{a}$ and $4.4 \mathrm{~b}$ demonstrate the average probability of packet reception by an arbitrary node, for varying one-hop success probability $p_{T}$, with the transmission range $R=0.6 \mathrm{~km}$, after $b=1,3$ rebroadcasts in a network of $N=10$ and $N=50$ nodes, respectively. The changing flow of these graphs implies that the rebroadcast steps are more forceful in dense settings of vehicles. It is because in rather a sparse network a very limited number of vehicles can receive the packet after each rebroadcast step and accordingly, there is no significant difference in the mean probability to own the packet. Therefore, we conclude the positive effect of the number of nodes, in other terms network connectivity, on the overall performance. Whereas, for fewer nodes, it is the success rate making the main contribution to the performance improvement as a result of increasing 
the rebroadcast steps. On the other hand, considering Figures $4.4 \mathrm{a}$ and $4.4 \mathrm{~b}$, one may note that how a higher success probability $p_{T}$ prevails the number of rebroadcast steps, leading to converging graphs for different number of rebroadcasts. This is because either most of the nodes (see Figure 4.4a) or even all of them (see Figure 4.4b) already receive the packet by the first rebroadcast step, with probability $p_{T}$. Hence, increasing the number of rebroadcast steps causes little difference in the outcome probability, particularly for higher values of $p_{T}$.

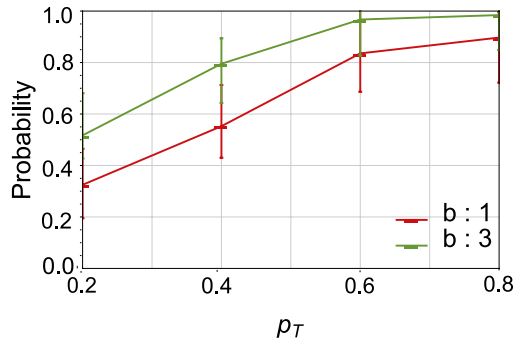

(a) $N=10$.

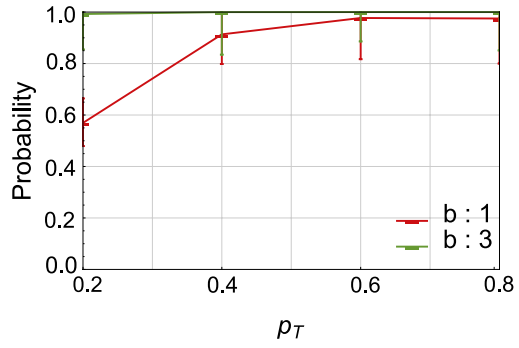

(b) $N=50$.

Figure 4.4: Reliability for varying $p_{T}, b, R=0.6 \mathrm{~km}$.

The confidence intervals shown in Figures $4.4 \mathrm{a}$ and $4.4 \mathrm{~b}$ indicate the spread of PDR outputs for various experiments.

We take a closer look at the spread of values for an example numerical setting in Figure 4.5. Corresponding topologies for upper and lower extremevalued experiments for $p_{T}=0.6$ are presented in this figure. One may note that the considerable difference between different experiments of the same numerical setting emphasizes the relevance of network graph characteristics. Note that in this case ten experiments are presented to avoid excessive overlap of the results.

Figures $4.6 \mathrm{a}$ and $4.6 \mathrm{~b}$ demonstrate the average probability of packet reception by an arbitrary node in a network of increasing number of nodes with transmission ranges of $R=0.3,0.6,1.2 \mathrm{~km}$, the number of rebroadcast steps set to $b=3$, with the success rates $p_{T}=0.4$ and $p_{T}=0.8$, respectively. These Figures imply a faster growth with larger $R$ and, as expected, the higher the $R$, the higher the effect of network density on overall probability improvement. These observations are in line with the observations shown in Figure 4.4.

Figures $4.7 \mathrm{a}$ and $4.7 \mathrm{~b}$ demonstrate the average probability of packet reception by an arbitrary node with an increasing transmission range $R$ in a network of $N=10$ nodes and success rates $p_{T}=0.2,0.4,0.8$, for $b=1$ and $b=3 \operatorname{step}(\mathrm{s})$ of rebroadcast, respectively. Comparing these figures, we note the positive impact of a higher number of rebroadcast steps on the output probability metric. 


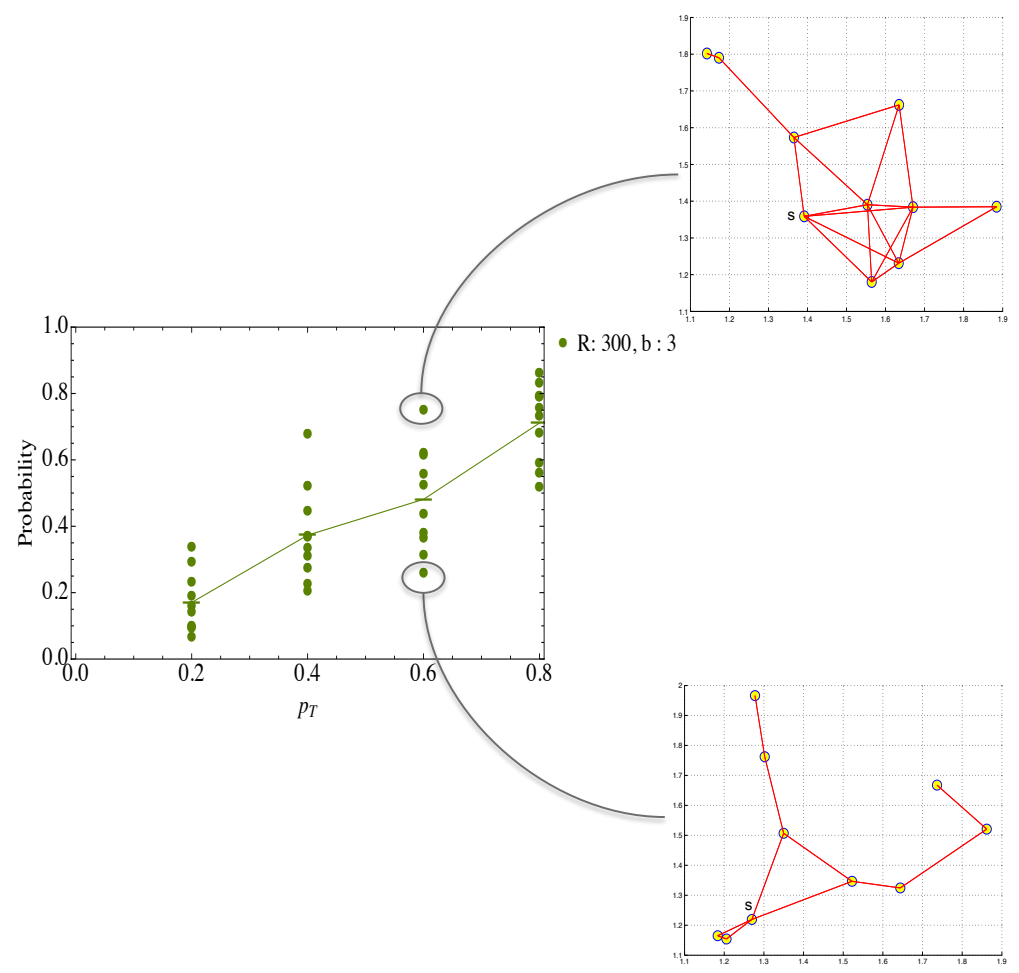

Figure 4.5: Reliability for varying $p_{T}, N=10$.

That is, by increasing $b$ higher probability is achieved with lower transmission ranges. In Figures $4.7 \mathrm{c}$ and $4.7 \mathrm{~d}$, we see the same graphs, but for $N=50$. Besides confirming the previous observation, comparing Figures $4.7 \mathrm{a}$ and $4.7 \mathrm{c}$, and also Figures $4.7 \mathrm{~b}$ and $4.7 \mathrm{~d}$, we note that increasing the success rate $p_{T}$ is more influential the sparser the network becomes. In the graphs, we can track a remarkable improvement of the output probability metric in the course of increasing the transmission range, particularly when it is supported by either a higher success rate and / or network density. Further, for $b=1$ step of rebroadcast, the success rate $p_{T}$ and number of nodes $N$ have similar effects on the overall output probability. However, for $b=3$ steps of rebroadcast, network density has a far greater effect on performance improvement. Slowly growing linear to constant curves for rather higher transmission ranges, imply the fact that most of the nodes are already accessible by this point. As expected, reacha- 
bility is achieved with lower transmission ranges in a denser network. In Figures $4.7 \mathrm{~b}$ and $4.7 \mathrm{~d}$ the situation is improved after three steps of rebroadcast, to the extent that we see a rather high dip linear increase in Figure $4.7 \mathrm{~b}$ and a sharp jump to the highest probability by a low transmission range $R=0.4-0.6 \mathrm{~km}$ in a dense network as shown in Figure 4.7d.

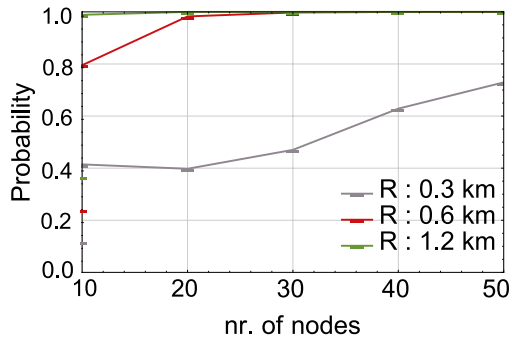

(a) $p_{T}=0.4$

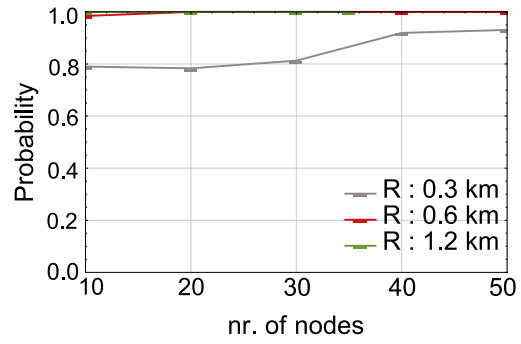

(b) $p_{T}=0.8$.

Figure 4.6: Reliability for varying $\mathrm{nr}$. of nodes and $R, b=3$.

In Figure 4.8, we consider a sample setting of $N=10$ nodes to track the impact of influential metrics as the success rate $p_{T}$, the transmission rate $R$ and the number of rebroadcast steps $b$, along with two corresponding topologies. Figures $4.8 \mathrm{a}$ and $4.8 \mathrm{~b}$ show the same layout of 10 nodes, but with different transmission ranges $R=0.3 \mathrm{~km}$ and $R=2 \mathrm{~km}$, respectively and accordingly different network diameters. That is, the maximum of all the shortest paths in the network. Now, we focus on the effect of increasing $p_{T}$ for these two cases whereas the number of rebroadcast steps is limited to $b=1$ and $b=3$, as demonstrated in Figures 4.8c and 4.8d, respectively. Besides the improving effect of a larger transmission range, it can be inferred from the graphs that for higher $p_{T}$, the difference between output probabilities for fewer and more rebroadcast steps is absolutely more considerable for lower transmission ranges than larger transmission ranges, as they diverge. Visible from the topologies, one may note that in a less connected network (Figure 4.8a), the number of rebroadcast steps has a higher impact on accessibility and accordingly reliability, due to the additional coverage provided by each rebroadcast. However, in a more connected network (Figure 4.8b) most of the nodes are already accessible from any other node in the network, regardless of the number of rebroadcast steps (most of the nodes are already covered by the first step of broadcast). It underlines the crucial role of the number of the rebroadcast steps in sparse networks of vehicles. This observation is inline with results published in [132]. 


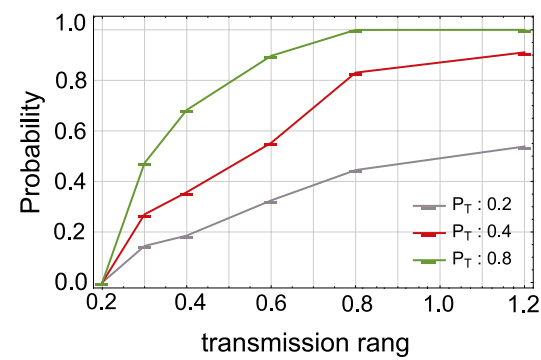

(a) $b=1, N=10$

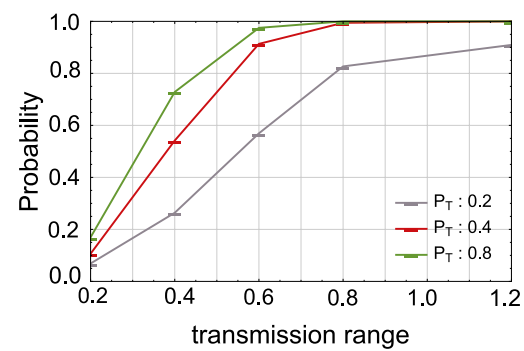

(c) $b=1, N=50$

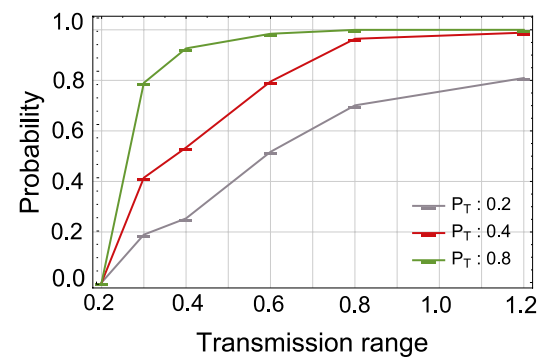

(b) $b=3, N=10$

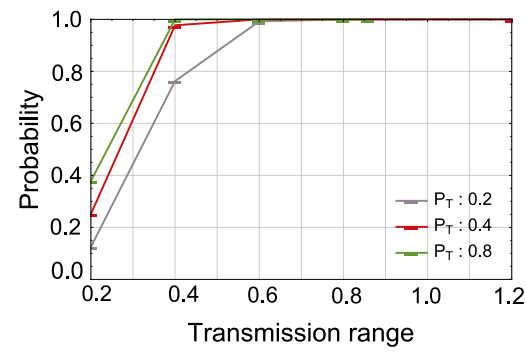

(d) $b=3, N=50$

Figure 4.7: Reliability for varying $R$ and $p_{T}$.

To further investigate the impact of the network diameter on the output probability as can be seen in Figure 4.8, Figures 4.9a and 4.9b demonstrate the average probability of packet reception by an arbitrary node in a network of $N=10$ nodes against increasing network diameter, for two extreme success rates $p_{T}=0.2$ and $p_{T}=0.8$, respectively. Transmission ranges are set from $R=0.3 \mathrm{~km}$ to $R=2 \mathrm{~km}$ with $50 \mathrm{~m}$ distance. We run twenty experiments with different topologies for each specific transmission range and later take an average over all PDR outputs belonging to identical diameters, spanning over all transmission ranges, along with the confidence intervals. These graphs support the aforementioned observation regarding the scale of the impact of higher rebroadcast steps in sparse networks. Further, it is noticeable that in a highly connected network (i.e., a lower network diameter) with a lower success rate (see Figure 4.9a), increasing the number of rebroadcast steps makes a bigger difference rather than in a less connected network (i.e., a higher network diameter). However, this is the other way around for higher success rates. That is, in sparse networks there is a larger gap between the probabilities corresponding to different number of rebroadcast steps. One may note that in a highly connected network as almost all nodes are accessible by the first rebroadcast step, 


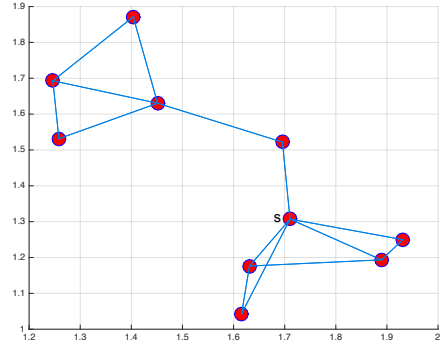

(a) $R=0.3 \mathrm{~km}$

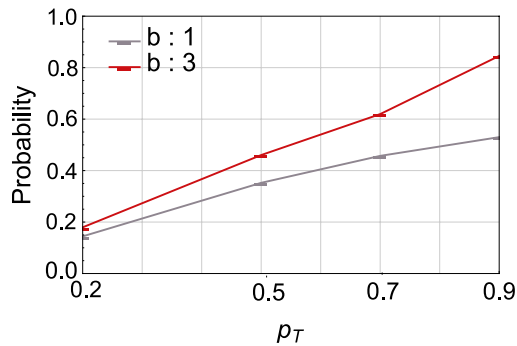

(c) $R=0.3 \mathrm{~km}$

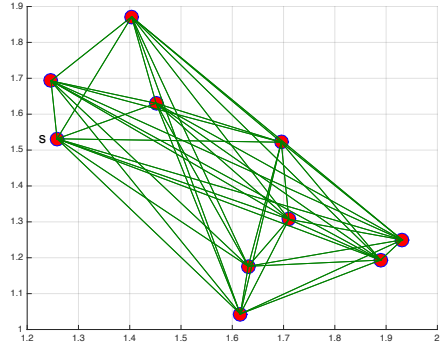

(b) $R=2 \mathrm{~km}$

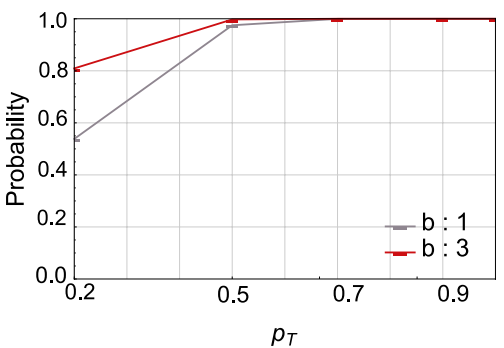

(d) $R=2 \mathrm{~km}$

Figure 4.8: Reliability for varying $p_{T}, N=10$.

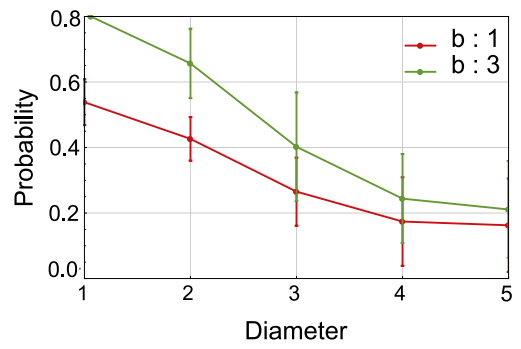

(a) $p_{T}=0.2$.

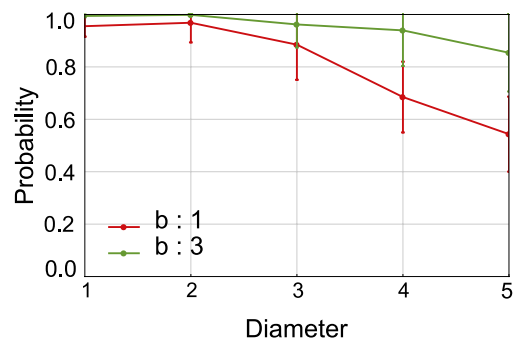

(b) $p_{T}=0.8$.

Figure 4.9: Reliability for varying network diameter, $N=10$.

the success rate is more outstanding while increasing the number of rebroadcast steps, influencing the resulting probability. That is why in Figure 4.9a with a lower success rate, the outcome probability for $b=1$ is considerably less than for $b=3$. However, in Figure $4.9 \mathrm{~b}$ with the success rate $p_{T}=0.8$, the outcome probability is almost the same for lower network diameters. 
Obtaining the same metric against the network diameter, this time changing as a result of varying number of nodes $N$ (instead of varying the transmission range $R$ ), we observe an interesting fact. That is, the outcome probability for a certain network diameter is roughly the same, reflecting the independence of the outcome probability from the factor deriving the change in the network diameter. In other words, this result emphasizes the network diameter as a major descriptive network characteristic to be taken into account in performance analysis.

Figures 4.10a, 4.10b and 4.10c demonstrate the average probability of packet reception by an arbitrary node over an increasing number of rebroadcast steps $b$, for the transmission range $R=0.3,0.6 \mathrm{~km}$, the success rate $p_{T}=0.2,0.4,0.8$ and the number of nodes $N=30,50$. Comparing these figures, one may note the difference in the additional coverage provided by more rebroadcasts in scenarios with different transmission ranges. Note that in rather sparse networks as shown in Figure $4.10 \mathrm{~b}$, with a very low success rate $p_{T}=0.2$, the output probability does not improve beyond a certain limit, despite more rebroadcast steps. However, a denser network as shown in Figure 4.10c compensates for a low success rate.

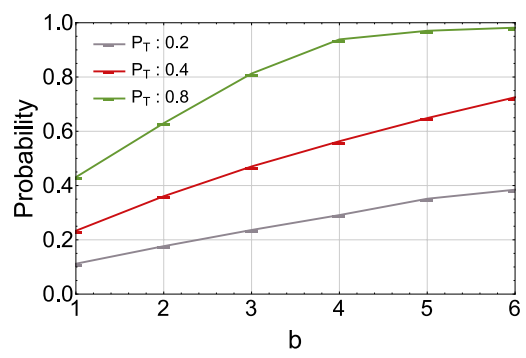

(a) $R=0.3 \mathrm{~km}, N=30$.

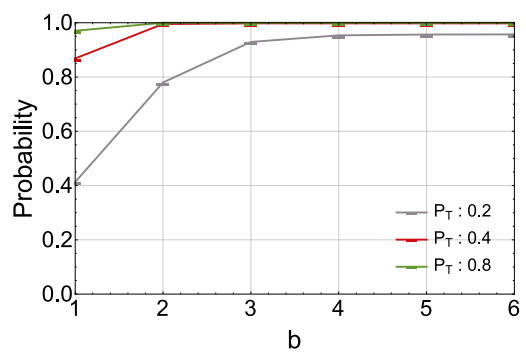

(b) $R=0.6 \mathrm{~km}, N=30$

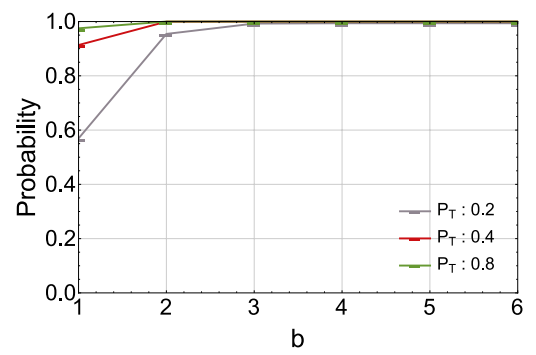

(c) $R=0.6 \mathrm{~km}, N=50$

Figure 4.10: Reliability for varying $b$ and $p_{T}$. 


\subsection{Error recovery}

Recalling from Section 4.2 on modeling the probabilistic multi-hop broadcast, at the end of a given number of rebroadcasts $b$, there would be nodes failed to successfully receive the broadcast packet. Assuming such nodes detected the loss using the reliability mechanism, described earlier in Chapter 3, they are supposed to broadcast a retransmission request for each lost packet. We call this phase, after the end of the multi-hop rebroadcast (first phase), the error recovery (second phase). The focus of this section is on modeling this phase of operation and later analyzing its performance on providing reliable delivery of data, with a reference to some work of relevance first.

\subsubsection{Related work}

Unreliability of vehicular environments makes it quite challenging to guarantee E2E (geo-)broadcast reliability, particularly when it comes to multi-hop communication. In this section, we mainly remark some work regarding reliability improvement of multi-hop broadcast.

Klein Wolterink et al. proposed Constrained Geocast in [150] which targets the vehicles based on their future and not the current position. In [40] authors proposed a spatiotemporal multicast/geocast protocol. Besides the geographic region, the time aspect is also considered in this approach, such that the geocast message is transmitted to all vehicles which are in the specific geographic region of interest at time $t$. Dynamic Time-Stable Geocast (DTSG) protocol [109] improves reliability by keeping the geocast message alive for some adjustable time in the geocast region. Geocache [85] proposed by Lakas et al. is a peer-to-peer pull-based geocast protocol which improves reliability by allowing vehicles to cooperatively collect and disseminate data. A caching mechanism is also considered, which reduces the amount of information exchanged between vehicles. Direction based Geocast Routing Protocol (DG-CastoR) [31] is based on link availability estimation for routing and considers the time factor in defining geocast regions by creating a virtual community, called rendezvous group, between mobile nodes that are able to communicate in a near future.

Yang et al. proposed in [154] a cooperative repetition by piggybacking old messages to the new ones as a means of improving reliability. The authors in [136] proposed a cross-layer decision based routing by taking performance measures from PHY and Medium Access Control (MAC) layers (e.g., Signal to Noise Ratio (SNR) and Signal to Interference plus Noise Ratio (SINR)) into the Network layer to calculate the communication range in order to optimize routing and accordingly improve reliability. Hassanabadi et al. proposed Synchronized Persistent Coded Repetition (SPCR) algorithm [72] as the main functionality of 
the reliability sub-layer in the application layer of the WAVE stack to increase the reliability of safety applications. That is rebroadcast of network coded messages. Simple and Robust Dissemination (SRD) approach [114] proposed by Schwartz et al. utilizes different strategies depending on the network density such that in dense networks the minimum number of nodes are assigned to relay the message (i.e., by means of broadcast suppression techniques) and in sparse networks the store-carry-forward model is used to deliver messages whenever multi-hop connectivity among vehicles is not available.

Cha et al. proposed Grid-based Predictive Geographical Routing (GPGR) [37] in which node positions are predicted during the relay node selection procedure as a means of increasing reliability. The authors in [61] based dissemination reliability on network connectivity. They proposed an analytical model to predict the number of nodes in the neighbourhood and accordingly adjust the probability of rebroadcast for a better coverage. Enhanced Multi-Hop Vehicular Broadcast (MHVB) [97] approach is flooding based and uses an algorithm to choose the right node to rebroadcast and subsequently increase reliability. In [100] authors compared three multi-hop broadcast algorithms as simple flooding, direction-aware flooding, and multi-point relaying. Though simple flooding outperforms the other two in terms of delay and delivery ratio, none of them has a explicit error recovery implemented. In [120] an E2E geocast acknowledgement scheme is proposed, in which individual ACKs are accumulated into larger messages in an aggregator and further forwarded back over multiple hops to the original source.

In summary, most of the related work result in implicit delivery reliability, by considering some rebroadcast decision making mechanisms. Works with explicit reliability schemes, either mostly do not rely on accurate information regarding necessity of error recovery from the receiving side or rely on, probably unnecessary, overloading acknowledgments. The reliability mechanism we analyse in this section is based on on-demand error recovery from the receiving side, avoiding redundant traffic.

\subsubsection{E2E reliability mechanism}

Recalling from Chapter 3, the mechanism discussed in this section, is a receiverbased E2E reliability assurance for (geo-)broadcast in vehicular environments. It enables error recovery by implementing a sequence numbering and checking functionality at communicating parties, as shown in Figures 3.1 and 3.2 in Section 3.3 of Chapter 3.

Through this mechanism, on failure of receiving a packet, a retransmission request is broadcast to all one-hop neighbors. Accordingly, the neighbors possessing that missing packet will reply by retransmitting it. As a means of col- 
lision avoidance, due to concurrent requests and replies, back-off timers are considered prior to requests and replies. Note that request and reply packets are broadcast to the single-hop neighborhood and they would not be sent, if overheard during the back-off (i.e. other nodes send the same request or reply that is overheard by the node backing off to send it). For further detail regarding its functionality, refer to Section 3.3 of Chapter 3. It is worth to remind that here we assume that the underlying ETSI ITS standard geo-networking infrastructure [50] is functionally available, as the basis to implement such a reliability mechanism.

\subsubsection{Modeling approach}

As mentioned earlier, at the end of the multi-hop rebroadcast phase, we end up with a situation where some nodes may have and some other may not have a broadcast packet with some probability. Such probabilities are computed according to Eq. 4.8 in Section 4.2.2.2.

Let $n_{i} \in\{0,1\}$ denote the status of an arbitrary node $\{i \mid i \in 1,2, \ldots, N\}$ regarding having $\left(n_{i}=1\right)$ or not having $\left(n_{i}=0\right)$ a packet. We could reasonably model the process of error recovery after the end of the multi-hop broadcast as a discrete-time absorbing Markov chain, with the system state at time $t$ characterized as

$$
S(t)=\sum_{i=1}^{i=N} n_{i} \times 2^{i-1},
$$

where $\left\{n_{i} \mid i \in 1,2, \ldots, N\right\}$ would be the status of node $i$ at that time. Since the source node has the status 1 , in principle the number of states is $2^{(N-1)}$. However, due to the network topology constraints regarding node connectivities, in practice the state space would be less and different for each particular topology.

Figure 4.11 shows an example of the state space for $N=4$ nodes, with principally $2^{(4-1)}=8$ states. In the binary representation of the states in this figure, the leftmost position represents the source node. Since the nodes with status "0" may receive the packet, as a result of request / reply rounds of error recovery mechanism, the system jumps between the transient states. Clearly, the transition between states, in the direction where the status of any given node changes as $1 \rightarrow 0$, has logically zero probability to take place. At the end, the desired state, where all nodes have the packet, is the absorbing state $\alpha$ and once entered cannot be left. 


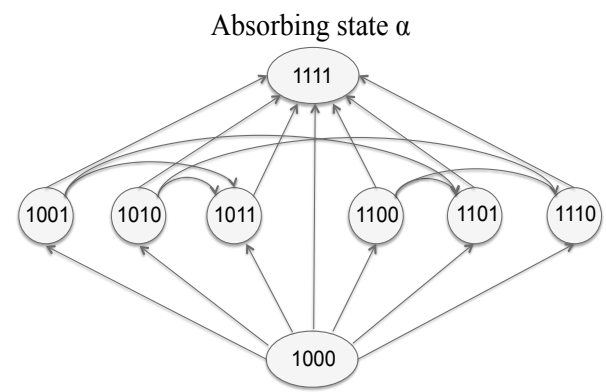

Figure 4.11: The Markov chain of 4 nodes, with binary state numbering.

An absorbing Markov chain with $M$ transient states and one absorbing state is canonically characterized by $[77]$

$$
T=\left[\begin{array}{cc}
Q & \vec{q} \\
\overrightarrow{0} & I_{1}
\end{array}\right]
$$

where $Q$ is an $M \times M$ transition probability matrix, with elements $\left\{q_{i j} \mid i, j=1,2, \ldots, M\right\}$, representing the probability of transition from the transient state $i$ to the transient state $j . \vec{q}$ is a $1 \times M$ absorbing probability vector, containing probabilities of direct transitions between the transient and absorbing state denoted as

$$
\vec{q}=\left(q_{1 \alpha}, q_{2 \alpha}, \ldots, q_{M \alpha}\right)
$$

Note that matrix $Q$ and its elements are not the same as matrix $Q_{T}$, described earlier in Section 4.2.2.2. $I_{1}$ is a $1 \times 1$ (because of one absorbing state) identity matrix and $\overrightarrow{0^{T}}$ is a $1 \times M$ vector of zeros.

The following condition must hold for an absorbing Markov chain, given by Eq. 4.10.

$$
\vec{q}+Q \vec{e}=1
$$


That is, the probabilities of leaving any arbitrary system state to other states or staying at the same state, all should sum up to one, where $\vec{e}$ is a vector of ones of size $1 \times M$.

Besides $Q$ and $\vec{q}$, a $1 \times(M+1)$ vector $\vec{P}$ of the initial probability distribution of system states, is needed to fully parameterize an absorbing Markov chain.

$$
\vec{P}=\left(P_{\alpha}, P_{1}, \ldots, P_{M}\right)
$$

In our case, it includes the probabilities of ending up in either of the system states (including the absorbing state with probability $P_{\alpha}$ ), after the end of the multi-hop rebroadcast (first phase). These probabilities could be derived from Eq. 4.8.

The Hamming weight (i.e., the number of nodes with the status "1") of an arbitrary state $i$, denoted by $H(i)$, is the number of potential replying nodes in that state and accordingly, $N-H(i)$, denoted by $F(i)$, is the number of potential requesting nodes (with the status " 0 ") in the same state. The Hamming distance between two states $i$ and $j$ (with logically non-zero probability of transition), denoted by $H D(i, j)$, shows the number of " $0 \rightarrow 1$ " changes. In other words, it shows the number of nodes that received a packet, as a result of transition from state $i$ to $j$. Given this, we aim to find the probability of transition from arbitrary state $i$ to $j$, denoted by $q_{i j}$.

There is a difference between modeling multi-hop and single-hop communication scenarios. In a similar system with "single-hop" communication scenario, all nodes of a network are supposed to hear each other. Hence, no matter which particular nodes miss a packet, it suffices considering a discrete-time absorbing Markov chain with the state space $\{S(n) \in 0,1, \ldots, N\}$, where the state number $S(n)$ describes the number of nodes not yet having a packet by time $n$ [65] [66].

However, in a "multi-hop" setting not all nodes are in one-hop neighborhood of each other and for modeling we need to differentiate among nodes, based on their positions in the network. Hence, we explicitly consider:

(i) the set of node(s) sending request(s) among all the potential requesters, with the status " 0 ", in a particular system state (denoted by the random variable $A$ );

(ii) the set of node(s) receiving the request(s) among all the potential repliers, with the status " 1 ", in that system state (denoted by the random variable $B$ ); 
(iii) the set of node(s) replying back to the received request(s) (denoted by the random variable $C$ );

(iv) the set of node(s) receiving the reply(ies) among all the existing nodes with the status " 0 " (denoted by the random variable $D$ ), resulting in the corresponding $0 \rightarrow 1$ status turns and consequently, the changed system state.

One may note that these four random variables correspond to four steps of a transition from an arbitrary state $i$ to an arbitrary state $j$. Such randomness implies the fact that a transition might be achieved through various ways, depending on the instances of these random variables, as shown in the example in Figure 4.12. Each of these ways may result in a different transition probability which contributes to the overall $q_{i j}$ probability of transition from state $i$ to state $j$.

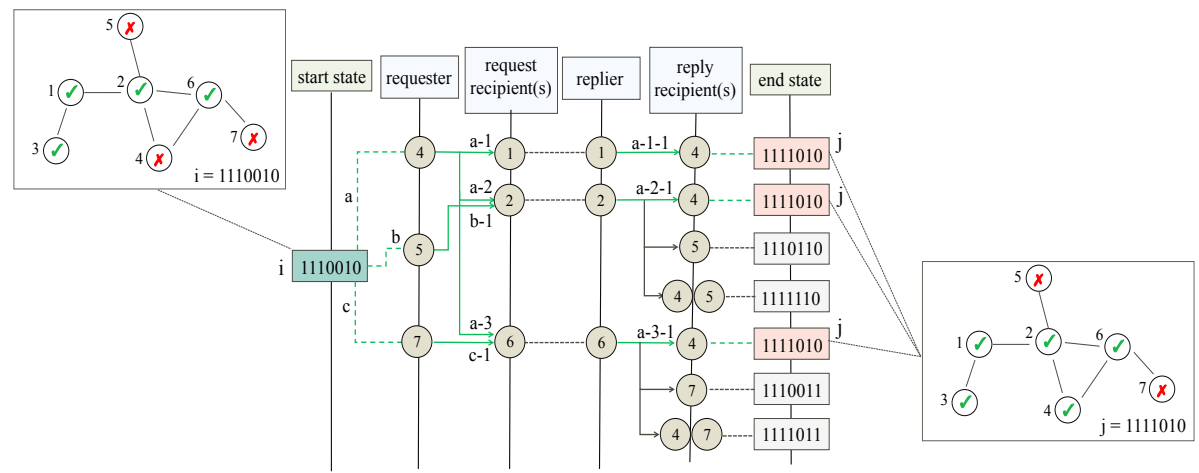

Figure 4.12: Transition from state $i=1110010$ to state $j=1111010$.

Recalling from the system state space and the Hamming weight property of states, we attribute an arbitrary state $i$ to two vectors $\overrightarrow{r e p_{i}}$ of size $1 \times H(i)$ and $\overrightarrow{r e q_{i}}$ of size $1 \times F(i)$, representing the ID's of the potential repliers and requesters in state $i$ respectively, as follows.

$$
\text { State } i \Rightarrow\left\{\begin{array}{l}
\overrightarrow{r e p_{i}}=\left(r e p_{i_{1}}, r e p_{i_{2}}, \ldots, r e p_{i_{H(i)}}\right) \\
\overrightarrow{r e q_{i}}=\left(r e q_{i_{1}}, r e q_{i_{2}}, \ldots, r e q_{i_{F(i)}}\right) .
\end{array}\right.
$$


For instance, considering $i=1110010$ as a transient state of a network of $N=7$ nodes and labeling nodes from left to right, we have

$$
\text { State } i \Rightarrow\left\{\begin{array}{l}
\overrightarrow{r e p_{i}}=(1,2,3,6) \\
\overrightarrow{r e q_{i}}=(4,5,7) .
\end{array}\right.
$$

Further, we define vector $\overrightarrow{r e c_{i j}}$ of size $1 \times H D(i, j)$ (i.e., the Hamming distance between states $i$ and $j$ ), corresponding to the transition from state $i$ to state $j$. This vector represents the ID's of the nodes that have received the packet as a result of transition from state $i$ to state $j$ as

$$
\overrightarrow{r e c_{i j}}=\left(r e c_{i j_{1}}, r e c_{i j_{2}}, \ldots, r e c_{i j_{H D(i, j)}}\right) .
$$

Considering example states $i=1110010$ and $j=1111010$, the $\overrightarrow{r e c_{i j}}$ vector would be $\overrightarrow{r e c} \overrightarrow{i j}=(4)$. Since node 4 is the only node receiving the packet, as a result of transition from state $i$ to state $j$.

Due to the topology-dependent reachability limitations between nodes, the random variables $A, B, C$ and $D$ are conditionally dependent. That is, the identity of nodes that act at each step of a transition, depends on the identity of nodes that acted in the previous step. Bayesian Networks can capture such conditional dependencies. Given this, we define a Bayesian network, represented by the Directed Acyclic Graph (DAG) [101], shown in Figure 4.13.

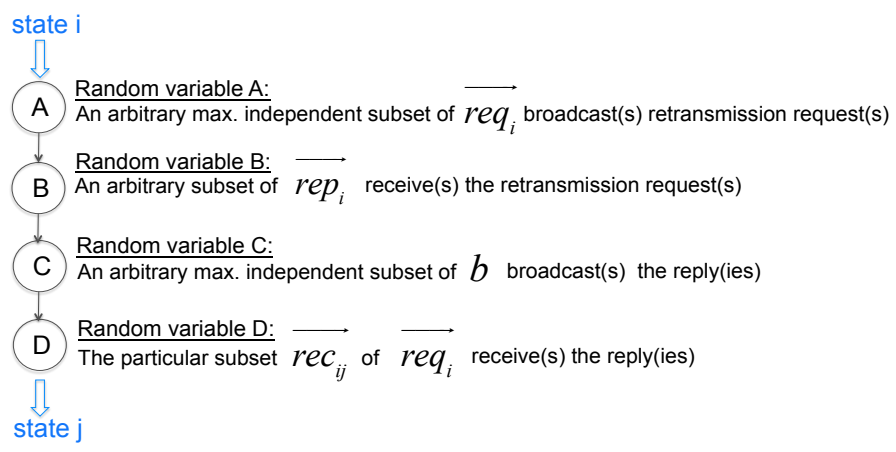

Figure 4.13: The DAG of the transition from state $i$ to $j$. 
The rationale behind considering "maximum independent" subsets in the first and third step, lies in the fact that according to the reliable (geo)cast protocol, neighboring nodes hear each other and do not send requests / replies for the same packet. This implies that in these steps, we just need to consider the independent subsets (i.e., pairwise non-adjacent nodes) of potential requesters /repliers, as the ones that may really send requests / replies for the same packet.

Following this, we have the joint probability of $A, B, C$ and $D$, by the chain rule of Bayesian networks [101] as

$$
P(A, B, C, D)=P(A) P(B \mid A) P(C \mid B) P(D \mid C) .
$$

Recalling the last step of the transition, we are interested in extracting $P(D=d)$ (denoted by $P(d)$ ) in Eq. 4.17, which is indeed $q_{i j}$. We can compute $P(d)$ from the joint probability by the method of marginalization [75], which is summing out variables $A, B$ and $C$. Accordingly, $P(d)$ is given by

$$
P(d)=\sum_{\forall a \in A} \sum_{\forall b \in B} \sum_{\forall c \in C} P(a) P(b \mid a) P(c \mid b) P(d \mid c) .
$$

By pushing the sums we have $P(d)$ as

$$
P(d)=\underbrace{\sum_{\forall c \in C} P(d \mid c) \underbrace{\sum_{\forall b \in B} P(c \mid b) \underbrace{\sum_{\forall a \in A} P(b \mid a) \underbrace{P(a)}_{\mathrm{P}(\mathrm{a})}}_{\mathrm{P}(\mathrm{b})}}_{\mathrm{P}(\mathrm{c})}}_{\mathrm{P}(\mathrm{d})}
$$

This is computationally less complex and could be solved by an inside-out dynamic programming method. In what follows, we calculate $P(a), P(b \mid a), P(c \mid b)$ and $P(d \mid c)$ in four steps. 
Step 1. $\mathbf{P}(\mathbf{a})$ This is the probability of a given Maximum Independent Subset (MIS) $a$ of nodes in $\overrightarrow{r e q_{i}}$, to be the requesters for a missing packet. We reasonably assume that in practice all the nodes that miss a packet and are pairwise far enough not to hear each other, will send a request for that packet. This implies that only the nodes in a MIS of nodes in $\overrightarrow{r e q}$ will send such a request. Considering all the MISs are equally likely to be chosen, the probability $P(a)$ is given by $1 / n r\left(M I S\left(\overrightarrow{r e q_{i}}\right)\right)$, where $n r\left(M I S\left(\overrightarrow{r e q_{i}}\right)\right)$ represents the number of all MISs of $\overrightarrow{r e q}_{i}$.

Step 2. $\mathbf{P}(\mathbf{b} \mid \mathbf{a})$ We define the following two probabilities for a given node $n \in \overrightarrow{r e p_{i}}$, to either belong to the subset $b$ or its complement $b^{\prime}$ related to $\overrightarrow{r e p_{i}}$.

$$
P(n \in b)=1-\left(1-p_{T}\right)^{\left|a_{n}\right|},
$$

where $\left|a_{n}\right|$ represents the cardinality of the set of nodes in $a$ that can reach node $n$. Such probability implies that node $n$ should receive a request, at least from one of the nodes in $a_{n}$. Accordingly, the probability $P\left(n \in b^{\prime}\right)$ is given by the following equation.

$$
P\left(n \in b^{\prime}\right)=\left(1-p_{T}\right)^{\left|a_{n}\right|} .
$$

Consequently, $P(b \mid a)$ is given by

$$
P(b \mid a)=\prod_{\forall x \in b} P(x \in b) \prod_{\forall y \in b^{\prime}} P\left(y \in b^{\prime}\right),
$$

implying that the probability that only the nodes in the subset $b$ and no other node in $\overrightarrow{r e p}$ receive the request(s), sent by nodes in the subset $a$.

Step 3. $\mathbf{P}(\mathbf{c} \mid \mathbf{b})$ Inline with the assumption in Step 1, $P(c \mid b)$ is given by

$$
P(c \mid b)=1 / \operatorname{nr}(M I S(b)),
$$


where $n r(M I S(b))$ represents the number of all MISs of the subset $b$.

Step 4. $\mathbf{P}(\mathbf{d} \mid \mathbf{c})$ Similar to Step 2, in order to compute $P(d \mid c)$, we define the following two probabilities for a given node $n \in \overrightarrow{r e q_{i}}$, to either belong to the subset $d$ or its complement $d^{\prime}$ related to $\overrightarrow{r e q}_{i}$.

$$
P(n \in d)=1-\left(1-p_{T}\right)^{\left|c_{n}\right|},
$$

where $\left|c_{n}\right|$ represents the cardinality of the set of nodes in $c$ that can reach node $n$. Such probability implies that node $n$ should receive a reply, at least from one of the nodes in $\left|c_{n}\right|$. Accordingly, the probability $P\left(n \in d^{\prime}\right)$ is given by the following equation.

$$
P\left(n \in d^{\prime}\right)=\left(1-p_{T}\right)^{\left|c_{n}\right|} .
$$

Consequently, $P(d \mid c)$ is given by

$$
P(d \mid c)=\prod_{\forall x \in d} P(x \in d) \prod_{\forall y \in d^{\prime}} P\left(y \in d^{\prime}\right)
$$

where it implies the probability that only the nodes in $d$ and no other node in $\overrightarrow{r e q}$ receive the reply(s), sent by nodes in the subset $c$. Now, by substituting all the relevant terms in Eq. 4.19, we arrive at $P(d)=q_{i j}$ as follows.

$$
\begin{aligned}
P(d)=q_{i j} & =\sum_{\forall c \in C^{x}} \prod_{x=1}^{|d|} P(x \in d) \prod_{y=1}^{\left|d^{\prime}\right|} P\left(y \in d^{\prime}\right) \times \sum_{\forall b \in B} 1 / n r(M I S(b)) \\
& \times \sum_{\forall a \in A^{x=1}} \prod^{|b|} P(x \in b) \prod_{y=1}^{\left|b^{\prime}\right|} P\left(y \in b^{\prime}\right) \times 1 / \operatorname{nr}\left(\operatorname{MIS}\left(\overrightarrow{r e q}_{i}\right)\right) .
\end{aligned}
$$


$P(d)=q_{i j}$ in Eq. 4.27 is capable of capturing all paths maybe traversed through, during the transition. Note that in a given scenario the transition from a particular state $i$ to a particular state $j$ could be logically feasible (i.e., there is no $1 \rightarrow 0$ change in the status of any node). However, due to the reachability constraints $q_{i j}$ maybe just 0 , implying it is not practicable to transit directly from state $i$ to $j$.

The elements of the absorption vector $\vec{q}$ are derived similarly by considering $d=\overrightarrow{r e q_{i}}$ in Eq. 4.27. Given this, we have the main attributes of the Absorbing Markov Chain (AMC) and this completes the parameterization of the system.

\subsubsection{Performance analysis}

\subsubsection{Evaluation metrics}

The Key Performance Indicators used to assess the performance of the mechanism, are introduced as follows.

Complete delivery probability Considering the retransmission requestbased property of the introduced error recovery technique, it is relevant the probability of successful packet delivery to all receivers within $k$ retransmission steps. Hence, for the modeled AMC, we focus on the CDF of the number of retransmission steps $k$, until absorption. Note that for an AMC, such probability is given by a FPT distribution, interpreted as the number of steps, required to end up in the absorbing state [77]. Given this and denoting the random variable, describing the number of retransmission steps until absorption by $S$, the CDF of $S$ is given by

$$
F_{S}(k)=\operatorname{Pr}(S \leq k)=1-\vec{P}^{\prime}\left(Q^{k}\right) \vec{e},
$$

where $\vec{P}^{\prime}$ is the initial state probability vector $\vec{P}$, without $P_{\alpha}$.

PMF of the number of retransmission steps Besides absorption within a limited number of retransmission steps, it may be of interest as well, the probability of absorption in exactly a predefined number of retransmission steps. That is the PMF of the number of retransmission steps and obtained from CDF, 
as follows.

$$
\begin{aligned}
& f_{S}(0)=F_{S}(0), \\
& f_{S}(k)=F_{S}(k)-F_{S}(k-1), k=1,2, \ldots, K .
\end{aligned}
$$

Overhead of error recovery Based on the fact that this receiver-based error recovery mechanism is built up on retransmission requests, it is also relevant to know the imposed overhead of such a scheme and investigate how many retransmission steps $k$ are to be done in a given scenario, for all the nodes to successfully receive a packet. This is given by the following equation.

$$
H=\sum_{k=1}^{\infty} f_{S}(k) \cdot k .
$$

It is interpreted as the expected number of retransmission steps, obtained by taking average over probabilities of absorption, for all the given number of retransmission steps $k$.

Residual loss probability In order to keep a reasonable trade-off between the overhead and improved reliability, it is also equally important to keep track of the system failure, in the case of the limited number of allowed retransmission steps. For this, we define the residual loss probability metric. That is, the average probability for an arbitrary station to be failed, after $k=K$ retransmission steps, given by

$$
p_{\text {res }}^{K}=\frac{\sum_{i=1}^{M}\left|S_{i}(n=0)\right| \cdot P_{i}^{K}}{M}
$$

where $\left|S_{i}(n=0)\right|$ is the cardinality of the set of nodes with status "0" in state $i$ and $P_{i}^{K}$ is $i^{t h}$ element of vector $\vec{P} \cdot T^{K}$, representing the probability of being in state $i$ after $k=K$ retransmission steps.

\subsubsection{Numerical results}

The results of evaluating the performance of the system, in terms of the introduced metrics, are presented here. First, we implement the analytical model 
and validate it using simulations and then we continue analyzing the system in detail, relying on the validated analytical model. Key system parameters such as the number of nodes, successful packet transmission probability, the transmission range and the number of retransmission requests are considered as the inputs to the analytical model. The results for each numerical setting are obtained by averaging over twenty random topologies of a given number of nodes and transmission range, uniformly distributed in a $2 \mathrm{~km} \times 2 \mathrm{~km}$ twodimensional area. Note that the rebroadcasting probability $p_{r b}$ is considered 1 and accordingly the number of rebroadcasts $b$ in the first phase is considered equal to the number of nodes $N$, assuming each node rebroadcasts only once, unless it is explicitly mentioned. Also note that the number of retransmission steps $k$ refers to retransmission requesting steps in the error recovery phase, not to be confused with the number of rebroadcasts $b$ in the first phase. Logarithmic scales are used in some graphs, as a means of reasonable presentation.

We use NS-3 to validate our model. For this, we set up a multi-hop wireless network of nodes with constant positions. This is a reasonable setting, considering the dissemination time of a packet, which is in the order of milliseconds, with regard to velocity of moving vehicles. The propagation loss is assumed to be negligible. Probabilistic packet transmission loss and the reliability assurance mechanism are implemented at the application level on all nodes.

Figure 4.14a shows the results, obtained via simulations and the analytical analysis, as the overhead of error recovery, in terms of the number of retransmission steps, against increasing probability of successful packet transmission $p_{T}$, in a network of $N=10$ nodes with the transmission range $R=0.5 \mathrm{~km}$. As expected, overhead follows a descending flow by an increase in $p_{T}$ and the two curves follow each other closely. Figure $4.14 \mathrm{~b}$ shows the results, obtained via simulations and the analytical analysis, for the residual probability of loss in a network of $N=10$ nodes and $R=0.5 \mathrm{~km}$. This probability decreases at the expense of more retransmissions. In particular, we observe a sharper decrease supported by a higher probability of successful packet transmission $p_{T}=0.98$. The results obtained from simulations are inline with the analysis, being within the confidence limits. Though they slightly fall apart, due to practical implementation differences between platforms and simplifications of the mathematical modeling. Relying on these observations, confirming the validity of the analytical modeling, in the rest of the paper, we merely consider the analytical model.

Figure 4.15 demonstrates CDF and PMF of the number of retransmission steps, until all stations receive the packet in a network of $N=5$ nodes, during the error recovery phase, for different packet success probabilities, $p_{T}$. Figure $4.15 \mathrm{c}$ and $4.15 \mathrm{~d}$ demonstrate the same metrics for $N=10$ nodes. For both $N$, we observe that PMFs are more evenly distributed for lower success probabilities, confirming necessity of more retransmission steps to be carried out to end up 


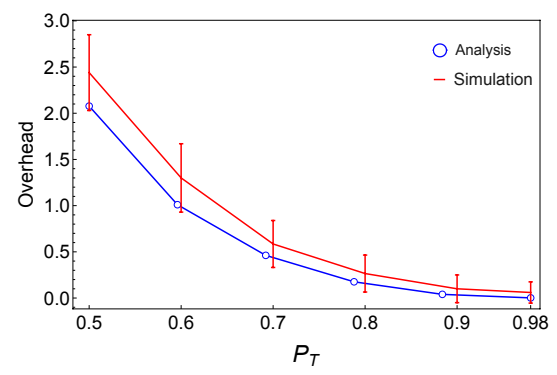

(a) Overhead of error recovery.

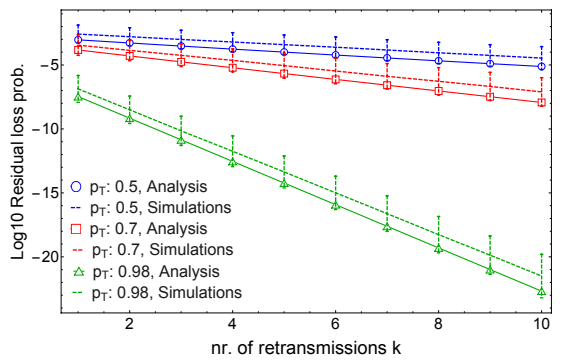

(b) Residual loss probability.

Figure 4.14: Validation test cases.

in the absorbing state, where all nodes have successfully received the broadcast packet. Comparing the peer graphs for $N=5$ and $N=10$, we follow a faster decrease in PMF values, against increasing number of retransmission steps, for $N=10$. This indicates the positive effect of increased network density on system absorption. The common remark regarding Figure $4.15 \mathrm{~b}$ and $4.15 \mathrm{~d}$ is the lightweight tail distribution of probability of absorption over the number of retransmission steps. This could be a determinative knowledge for the trade-off between improved reliability and imposed overhead, in terms of the number of retransmission steps.

It is likely that all nodes successfully receive a packet at the initial rebroadcast phase. Such a probability $\left(P_{\alpha}\right.$ in $\left.\vec{P}\right)$, which is not included in Figure $4.15 \mathrm{~b}$ and Figure 4.15d, contributes to the overall probability of successful packet delivery to all nodes. Such that, adding this probability to the corresponding sum of PMFs of all the number of retransmission steps (i.e. CDF), sums up to 1, as it is supposed to be and shown in Figure 4.15a and Figure 4.15c. The same is valid for all the other experiments.

In Figure 4.16, we see the effect of the number of rebroadcasts $b$ in the first phase (i.e. the multi-hop rebroadcast), on the error recovery (second phase) performance, for the transmission range $R=0.5 \mathrm{~km}$ and the packet success probability $p_{T}=0.5$. These figures demonstrate CDF and PMF of the number of retransmission steps $k$, until all stations successfully receive the packet, during the error recovery phase, given different number of rebroadcasts $b$, in the first phase.

These observations are mainly useful in the case of applying probabilistic rebroadcasts in the first phase. For instance, we see no big difference between results, belonging to $b=3$ and $b=10$ number of rebroadcasts. Hence, the 


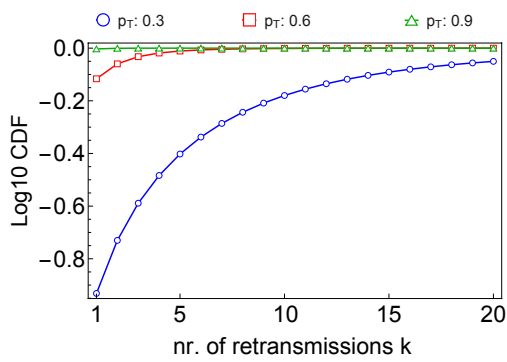

(a) $C D F, N=5$

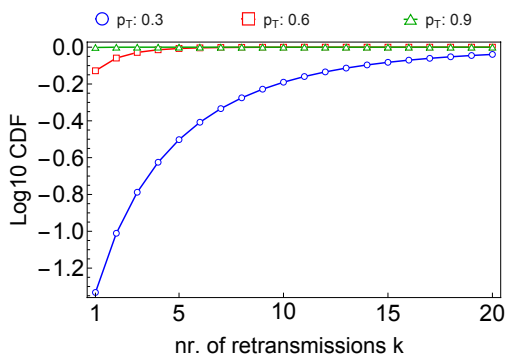

(c) $C D F, N=10$

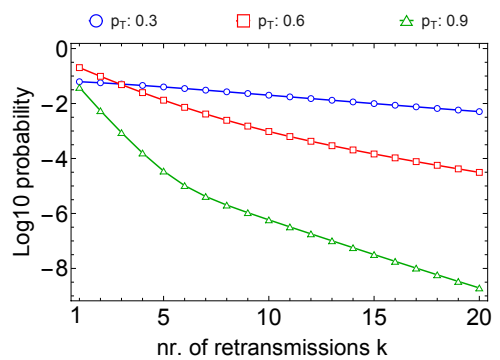

(b) $P M F, N=5$

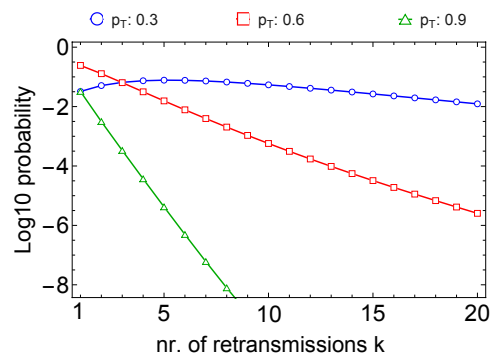

(d) $P M F, N=10$

Figure 4.15: CDF and PMF of the number of retransmission steps for different probabilities, $N=5,10$.

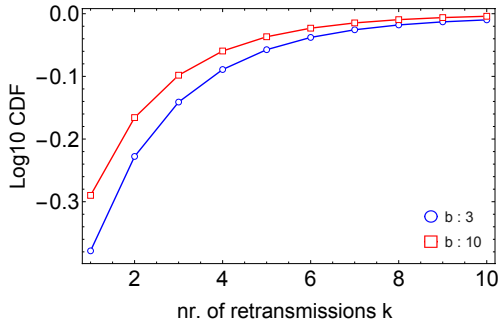

(a) $C D F, N=10$

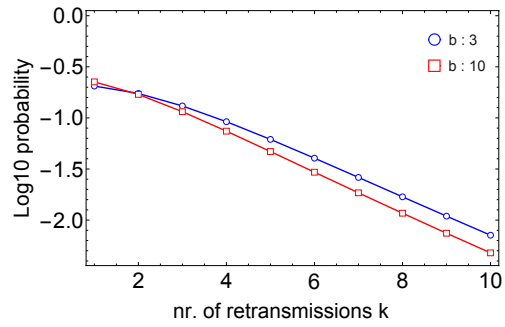

(b) $P M F, N=10$

Figure 4.16: $\mathrm{CDF}$ and PMF of the number of retransmission steps for $p_{T}=0.5$ and different rebroadcast steps, $b=3,10$.

rebroadcasting probability $p_{r b}$ could be adjusted, not to overload the network with excessive rebroadcasts. 


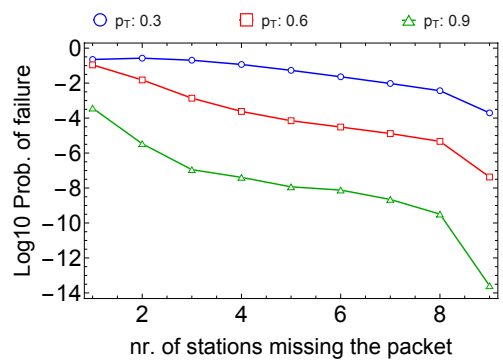

(a) \#retrans $=2$

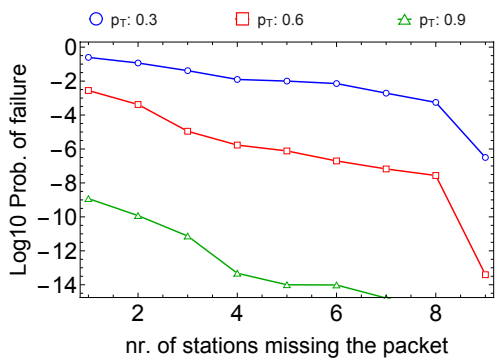

(b) \#retrans $=8$

Figure 4.17: Failure prob. after given retrans. steps, $N=10, R=0.5 \mathrm{~km}$.

Figure 4.17 demonstrates the probability that a given number of stations fail to receive the packet, after $k=2,8$ retransmission requests, for three different probabilities $p_{T}=0.3,0.6,0.9$. What is straight in these figures, is sharper decrease of the probability of failing having higher number of unsuccessful stations, for higher probability of success $p_{T}$. The same behavior, in a more intense scale, is observable while comparing Figures $4.17 \mathrm{a}$ and $4.17 \mathrm{~b}$. In particular, when supported by better channel conditions (i.e. for the higher $p_{T}=0.9$ ), increasing the number of retransmission steps $k$, results in way more significant improvement.

Figure 4.18a demonstrates the overhead of error recovery, in terms of the number of retransmission steps $k$ against increasing probability of successful packet transmission $p_{T}$, for three transmission ranges $R=0.3,0.5,0.8 \mathrm{~km}$, in a network of $N=10$ nodes. We note a smooth decay in the imposed overhead, as either $p_{T}$ or $R$ increases. The dominant $p_{T}$ factor, suppressing the gap between curves corresponding to various $R$, is traceable as the curves merges towards higher $p_{T}$ s.

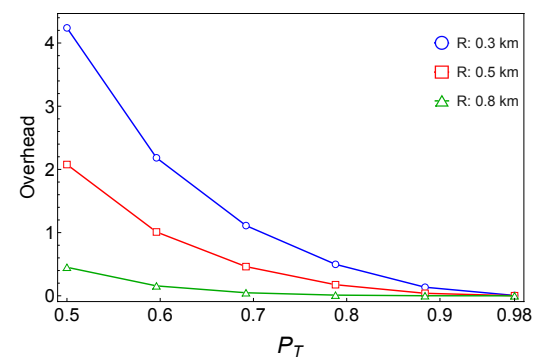

(a) Overhead of error recovery.

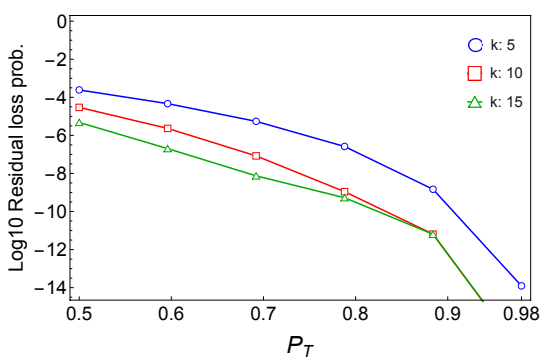

(b) Residual loss probability.

Figure 4.18: Recovery overhead and residual probability of loss, $N=10, R=0.5 \mathrm{~km}$. 
The residual loss probability is shown in Figure 4.18b for a network of $N=$ 10 nodes and $R=0.5 \mathrm{~km}$. Expected behavior of the system is confirmed by the model, as we see smaller probabilities of residual loss, against increase in $p_{T}$. Non-significant gap between curves, belonging to three different number of retransmission steps $k$, implies the fact that most of nodes are reachable by fewer number of retransmissions.

\subsection{Concluding remarks}

In this chapter, we analytically modeled and evaluated a reliability assurance mechanism in the context of multi-hop broadcast. For this, we first proposed an analytical model of two-dimensional multi-hop broadcast in Section 4.2. We defined the probability of broadcast packet reception by nodes of a network after a given number of (re)broadcast attempts, as a dissemination performance indicator and we quantified it, using the model. Further, considering major network characteristics, such as network density, nodes' transmission range and packet transmission loss probability, we investigated how they affect that output probability, by evaluating various numerical settings of the model. Such observations could be used in optimizing the flexible parameters, such as transmission range, relaying probability and the number of rebroadcast steps, in various network conditions.

The main strength of this model is that it takes into account the network topology in terms of connectivity graphs and consequently, the effect of major characteristics such as network density and the number of 1-hop neighbors. We also considered network graph characteristics and examined the impact of network connectivity schema on the output probability, while other numerical settings were not changing. Also, future broadcast optimization algorithms could benefit from the contributions of this work. As for instance, the proposed model could be used to derive the impact of an optimization algorithm on the defined performance measure, or a new measure introduced in the framework of the model.

In the numerical part of this work, we considered the rebroadcast probability $p_{r b}$ as 1 (e.g., simple flooding). Based on results in [132], one may note that decreasing $p_{r b}$ not only does not deteriorate the performance in rather dense networks, but also diminishes the broadcast storm effect by saving many redundant rebroadcasts. Besides probabilistic broadcasting, various mechanisms proposed in [132] could be utilized, with optimal settings for various scenarios, in conjunction with our work to achieve even better performance results from other perspectives, such as network load and collision avoidance. 
By taking the result of modeling two-dimensional multi-hop broadcast as input, for the next step towards improving the reliability, in Section 4.3 we focused on analytical modeling of the receiver-based error recovery scheme, introduced in Chapter 3, in the context of multi-hop broadcasting.

Using simulations, we proved the validity of the analytical model. Our observations are useful for designing relevant optimization schemes, for reliable data dissemination. For instance, parameters such as nodes' transmission range or, particularly in this case, the number of retransmissions could be tuned, given various network densities and channel conditions.

The results presented in Section 4.3.4.2, emphasize the fact that even though retransmission requests and replies are broadcast in single-hop, the proposed E2E reliability mechanism provides reliable delivery in multi-hop scale, with limited number of retransmission attempts. That is due to the hop-by-hop penetration of the request and reply messages sent by multiple nodes in the network.

The model is extensible such that elaborate $f(\theta)$ functions, considering detailed parameterization of data dissemination, can be augmented into the transition probability matrix that we developed.

Ensuring ultra reliable broadcast, using existing technologies [59] is not yet achieved. Motivated by the concept of heterogeneous networking, as the main design criteria for the next generation of the mobile networking system, in Chapter 5 we will investigate the potential gain of using cellular communication technologies, to contribute to reliable vehicular communications. 


\section{Resource allocation for cellular D2D vehicular broadcast}

In this chapter, we focus on the URLLC service of the next generation mobile networking system (i.e., $5 G$ ) as the basis for D2D broadcast communication, a promising enabler for broadcast-based Cooperative Intelligent Transport Systems (C-ITS) applications with strict reliability requirements. In particular, we look at the resource management aspect of D2D communication to contribute to reliable broadcasting. We propose a resource allocation scheme which is adaptive to the varying state of a vehicular network. By focusing on the network load and topology as the main criteria, our scheme aims for management of the system capacity and interference situations while allocating resources, in order to meet the performance requirement of D2D vehicular broadcast, in terms of reliability. The results confirm the effectiveness of our approach and provide insight on the optimal network design regarding the allowed data rate and resource assignment according to application requirements. The publication related to this chapter is [67].

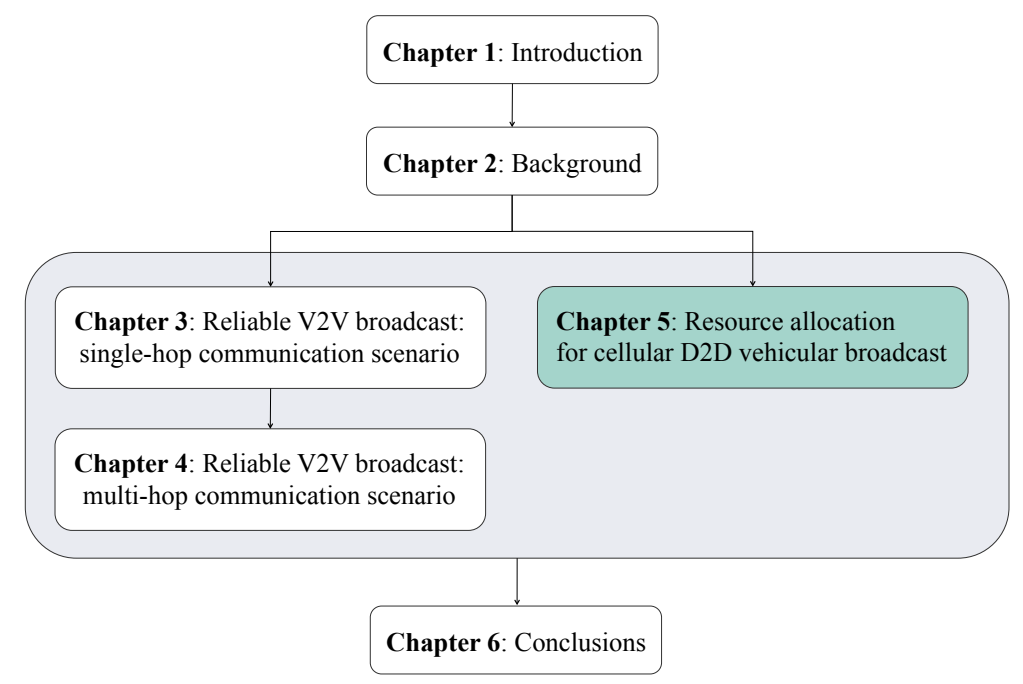


This chapter is organized as follows:

- Section 5.1 gives an introduction to D2D communication technology and its application for vehicular broadcasting; It also states the contribution of this chapter.

- Section 5.2 discusses relevant literature.

- Section 5.3 describes basic assumptions regarding resource allocation and the propagation model, as the preliminaries to our proposed resource allocation approach.

- Section 5.4 introduces our approach of adaptive resource allocation.

- Section 5.5 details the first modeling approach of consideration where our resource allocation scheme is applied in and further evaluated in terms of relevant performance indicators.

- Section 5.6 details the second modeling approach of consideration where our resource allocation scheme is applied in and further evaluated in terms of relevant performance indicators.

- Section 5.7 concludes this chapter. 


\subsection{Introduction}

Reliable wireless communication between vehicles is crucial for high-performance ITS applications. The current underlying technology falls short of supporting such demands. Accordingly, transformations in vehicular communications and the underlying technology are being discussed.

IEEE 802.11p is the main standard for vehicular communication, with the modified PHY layer from IEEE 801.11a standard based on Orthogonal Frequency Division Multiplexing (OFDM) and the MAC layer from IEEE 801.11e standard based on the EDCA. Despite desirable features such as fully distributed direct communication without network coverage and no need for a priori formation of a BSS for communication, it has shortcomings, mainly due to its intrinsic characteristics. IEEE $802.11 \mathrm{p}$ has scalability issues due to its contention-based medium access (i.e., CSMA/CA) from the EDCA protocol. Accordingly, its throughput performance decreases significantly under high network loads and a maximum latency cannot be guaranteed. Also, the probability of collision increases in high network densities, due to the hidden node problem and this results in high packet error rates. Decentralized Congestion Control (DCC) [51] is introduced in ETSI standards as a cross-layer function to address congestion by adjusting controllable parameters such as transmission power, frame duration and frame transmission rate based on the channel load. However, the exact relation between these parameters and the performance of the DCC algorithm is still an open issue, due to the lack of insight on the hidden node problem in Carrier Sense Multiple Access (CSMA) [124] [125]. Further, the behavior of DCC by reducing the packet transmission rate and thus reducing congestion may not be reasonable for safety applications where the packet throughput is important factor [128]. The short communication range is another limitative aspect of IEEE 802.11p, resulting in coverage extension issues. Although RSUs can be deployed to increase the communication range and also to provide the Internet access through infrastructure, their large-scale deployment is not economically viable, given the already existing coverage from the mobile networking system. Another bottleneck is the inefficient utilization of the $5.9 \mathrm{GHz}$ frequency band. This spectrum is split into channels of $10 \mathrm{MHz}$, with one of them considered as the safety channel (i.e., $\mathrm{CCH}$ ). However, such a fixed channel allocation is not suitable for the time-varying vehicular networks. In addition, IEEE 802.11p does not have a default acknowledgement technique for broadcast type of communication and as a consequence lacks reliability assurance. Finally, central security management of IEEE 802.11p, which is based on digital signatures, may result in significant delay, in particular for safety applications. The decentralized approach may also suffer from large overhead. 
Motivated by all these shortcomings, the cellular mobile network with a large-scale coverage, has recently been considered as an alternative for vehicular communication. Transmissions in such a system are scheduled by a network scheduler (i.e., eNodeB), hence avoiding collisions. This is crucial in particular for highly loaded networks, as a maximum delay can be guaranteed, given the central resource allocation and priority based admission control. With respect to security, utilizing the existing mobile networking system will facilitate management in terms of revocation of certificates, update procedures and lower overhead as a means of highly secure vehicular communication. However, the 3GPP cellular system is originally designed for mobile broadband communication, with specific requirements. Conventional cellular communications rely on the infrastructure. That is, all the traffic traverses through base stations (i.e., eNodeBs) in uplink and downlink. Though it has benefits such as mobility management, it may lead to inefficiencies mainly in terms of latency, scalability and energy consumption.

Research activities have been initiated in the recent releases of 3GPP LTE (i.e., the fourth generation cellular system $4 G$ ) towards incorporating features supporting new types of communication. In this regard, LTE sidelink (or D2D) communication was introduced for the first time in Release 12 (mainly for public safety applications), by adding new interfaces (PC3, PC4, PC5) and the component ProseFunction [21] into the legacy cellular network [89] [30] [21] [20]. As shown in Figure 5.1, the wide area network LTE interface (Uu) connects user devices to mobile network base stations for further connection to mobile core networks, for provision of Internet and other network management services. The direct communications interface (PC5) is considered for enabling direct discovery and communication of users in the proximity of each other without traversing the network infrastructure, for provision of low-latency and highreliability services. More details regarding the interfaces and the functionality of the component ProseFunction is out of the scope of this thesis.

Release 14 includes support for V2X communication by introducing LTE-V technology [24] based on D2D communication (also known as LTE-V2X and cellular V2X), with the enhanced PC5 interface [21] for vehicular use cases (i.e., high speed and high density). LTE-V predominates over the IEEE 802.11p standard, namely due to its large-scale coverage, high data rate, high penetration rate and high-level Quality of Service (QoS) support. Accordingly D2D-based vehicular communication is considered a promising approach to fulfill requirements of safety-critical vehicular applications (See [79] [141] [36] [143] [157] [156] for more details regarding performance comparison between two technologies). In this respect, URLLC as one of the three main services of the next generation (i.e., 5G) mobile networking system, targeting high reliability and low latency communications, is a good fit and accordingly considered a suitable service for 


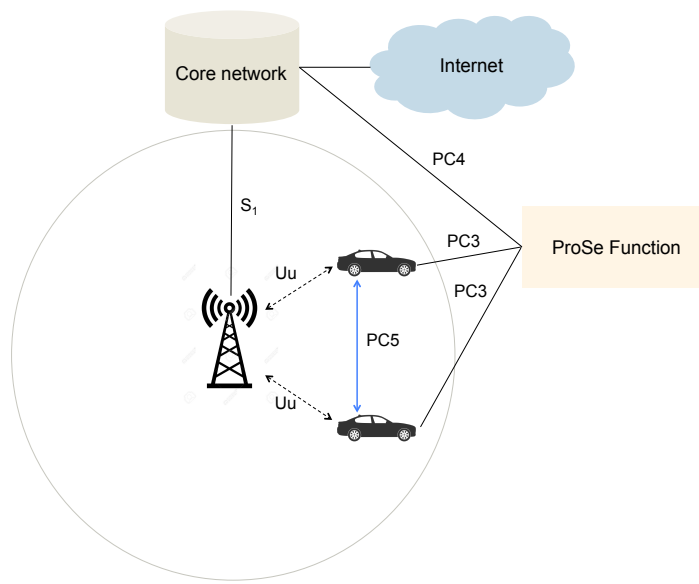

Figure 5.1: Direct (V2V) vs. infrastructure-based (V2I) communication modes.

D2D-based broadcast. The other two are extreme Mobile BroadBand (eMBB) and massive Machine-Type Communication (mMTC) [19]. As demonstrated in Figure 5.2, each of these services target performance indicators for different use cases, where the safety critical vehicular applications with strict latency and reliability requirements are proposed to be addressed by the URLLC service.

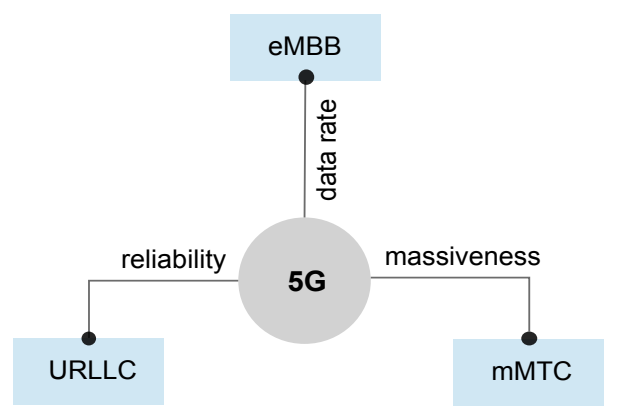

Figure 5.2: Main services of the fifth generation mobile networking system [19].

The availability of the cellular infrastructure as a central management entity, eliminates issues like collision and resource detection in D2D communications. On the one hand D2D communication benefits from scheduling capabilities of the eNodeB and on the other hand, D2D communication does not undergo two-hop uplink / downlink communication. This results in gains in terms of 
spectral efficiency, low latency, low transmission power and high data rate. In this respect, the resource allocation management can be differentiated into two modes as centralized (scheduled) and distributed (autonomous). In the first case, the cellular network assigns the radio resources for D2D communication, whereas in the second case users access them randomly on their own [22] from a dedicated resource pool. While the first mode requires cellular network coverage, the second mode does not necessarily rely on the network coverage.

Having an efficient way of managing resources, is an essential factor in realizing advantages of D2D communication. As shown in Figure 5.3, D2D communication can be classified into two main categories, from resource spectrum utilization point of view. One is out-band (i.e., using unlicensed bands for D2D transmission) which is out of the scope of this chapter, since we are interested in utilizing the scheduling capabilities of the cellular infrastructure. The other is in-band (i.e., using the licensed cellular spectrum for D2D transmission) which can be further divided into underlay and overlay schemes [29] [45]. The underlay in-band D2D refers to the case where the radio resources are shared between the cellular users and D2D users. In conventional cellular communication Resource Block (RB)s are mostly assigned on a per user basis, by the base station. This implies orthogonal (i.e., not interfering) resource allocation for users. The same way can be adopted for underlay D2D resource allocation, where only unused RBs are considered for allocation to D2D users. However, taking into account the ever-increasing number of users and scarcity of resources, efficient spectrum utilization and accordingly the reuse of the RBs come into prominence. This may lead to interference between transmissions using the same resources. As a means of avoiding interference, dedicated resources (i.e., resource pool) may be considered for D2D communication. This is called the overlay in-band D2D.

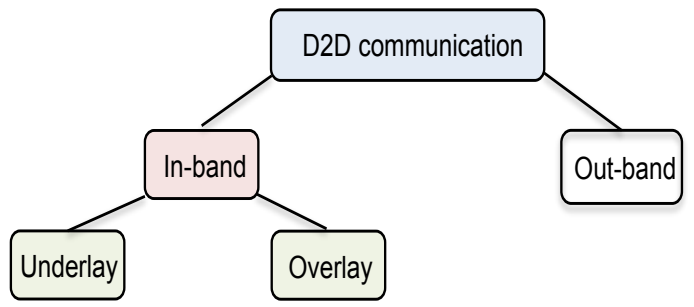

Figure 5.3: D2D classification.

There are different challenges in realizing resource efficient and reliable D2D communication. It is important to take into account aspects such as the system capacity and interference conditions, while allocating resources. The first aspect is about the problem of instantaneous network load management, given 
non-stationary demand for D2D communication. With regard to the second aspect, spatial resource reuse plays a crucial role. The problem is, while having many concurrent D2D communications is targeted as a means for better resource utilization, it may lead to increasing interference and accordingly performance degradation, in the case of resource reuse. All these imply the fact that a fixed resource allocation scheme cannot fit into the varying state of a vehicular network.

In this chapter, we study the application of D2D communication overlaying the cellular spectrum for vehicular broadcasting. By considering the current fourth generation of the mobile networking system as the baseline, we propose a D2D resource allocation scheme which is adaptive to the network load and topology in order to support reliable V2V broadcast.

The main contributions of this chapter are as follows:

- we propose a centralized resource allocation scheme for D2D vehicular broadcast, with adaptive functionality according to the varying network condition;

- we analyze performance of the proposed resource allocation scheme in the context of two modeling approaches, featuring different characteristics and application requirements. While one is focused on the system behavior over a rather long period of time, the other looks at a limited communication time;

- based on the developed models, we confirm the effectiveness of the proposed scheme via numerical results from both the system and end-user point of view.

\subsection{Related work}

The surge of study on heterogeneous networking brought along the focus on incorporating direct D2D communication in cellular networks and this, also supported by the promising gains, has led to significant amount of research activities in industry and academia. In this section we refer to some recent work of relevance to the fundamental aspect of resource management in D2D communication.

In [118] the authors considered a distributed resource allocation scheme and proposed a MAC protocol, where D2D transmitters contend for accessing data channels. However, the time needed to get access to the medium may give rise to scalability issues in dense scenarios, in particular for safety critical ITS applications. 
The authors in [106] proposed an underlay D2D resource and power allocation scheme in a two step joint optimization. The resources are allocated, conditioned on satisfaction of the minimum throughput of the D2D multicast groups. Accordingly, power allocation is done by applying an approximation of Shannon capacity. Though the authors proposed a less complex sub-optimal solution, full knowledge of Channel State Information (CSI) is assumed in order to compute the levels of SINR for all users which is not a reasonable assumption, due to the overhead and the amount of required time.

A similar work in [142] proposed a two step interference coordination scheme for a single cell, with the aim of improving system throughput and resource utilization. First, the upper bound of a D2D transmitter power is derived via a power control scheme. Then, given this upper bound, resource allocation rules are applied such that controlling the interference from cellular users to receivers in a single D2D multicast group. The authors considered all possibilities for resource allocation permutations and the corresponding level of SINRs. Though they proposed a sub-optimal version of the scheme with less complexity, it may not be tolerable the overhead of computing the interference level of each resource for all D2D users.

Sun et al. proposed in [129] an algorithm for resource sharing and power allocation in a two step optimization problem, by taking into account latency and reliability requirements of vehicular applications. Doppler et al. proposed in [45] power control by the base station as a means of interference management when a D2D transmitter reuses the cellular uplink resources. In [153] it is proposed using a MAC protocol where interference management is based on the Signal to Interference Ratio (SIR) and it is functioning similar to RTS/CTS in WiFi. Receivers measure the SIR of the received control signals by the transmitters and accordingly decide to respond to the one with the highest level of SIR. The work in [107] proposes an interference management approach where it is assumed the knowledge regarding resource allocation for all the cellular users is available both for other cellular users and also D2D users. That is, each D2D transmitter predicts the interference from all cellular users and accordingly selects for reuse the resource of a cellular users which results in the least interference. The authors in [35] proposed a location-based resource allocation scheme where a cell area is divided into non-overlapping sections and predefined set of resources are considered for each section. The resources of a section can be reused in another section with enough distance, as a means of interference management. However, such a fixed area division and resource grouping may not meet the dynamicities of the network and as a result resources can be inefficiently underutilized. In [91] it is proposed to define a set of transmission patterns and accordingly randomize multiple D2D broadcast transmissions over the time domain. As a means of transmission scheduling, it is assumed that 
each user obtains the information regarding the transmission patterns and path losses from all other users and chooses a pattern such that the resultant interference is less than a predefined threshold which is a computationally expensive approach. The scalability is another issue, in terms of the transmission patterns for higher number of users.

Most of the existing work assume the CSI of all links to be available at the base station, in order to efficiently manage allocation of resources. This is probably not a reasonable assumption, in particular considering the computation overhead for dynamic networks. Moreover, very limited work is available for overlay D2D and also the majority of the previous studies consider the radio resources to be reused by at most one other user and evaluate the performance of a single D2D pair. Whereas intelligent reuse of resources by as many as possible users will significantly improve spectrum efficiency. Arguable is also the scalability of the distributed resource management schemes.

Considering the aforementioned limitations, in this chapter we develop and analyze a dynamic mechanism of central resource management for D2D broadcast communication, overlaying the cellular network. For this, the base station does not rely on the CSI of the involved links. Rather, the location information of D2D transmitters are sufficient which is often available in ITS applications via Global Positioning System (GPS) data, hence lightening the overhead of control signaling. Dynamicity of our approach is two-fold, in terms of the network load and topology. To be more specific, we propose a resource allocation scheme, where first given the network load and system bandwidth, the number of obtainable resources, out of the available bandwidth are computed. Then, given the network topology, D2D transmitters are spatially grouped and assigned with resources, as a means of interference-controlled reuse of the allocated resources.

In what follows, we first provide in Section 5.3 the preliminaries. These include resource allocation basics and assumptions in Section 5.3.1 and the propagation model considered for broadcast in Section 5.3.2. We continue by introducing our resource allocation mechanism in Section 5.4. Section 5.5 and 5.6 detail two modeling approaches which we consider for evaluating the performance of our resource allocation mechanism.

\subsection{Preliminaries}

In this section, we present in detail resource block allocation preliminaries and assumptions from [22] as the foundation of our proposed resource allocation approach. Further, we present the data propagation approach used for broadcasting. 
Table 5.1 lists the common notation used in this chapter, unless otherwise mentioned later. If applicable, values are mentioned for each parameter. Note that notation and / or values specific to each modeling approach are introduced in the corresponding section.

Table 5.1: Notation used throughout this chapter.

\begin{tabular}{|c|c|}
\hline Parameter & Definition \\
\hline$B W$ & The bandwidth of the system $(10 \mathrm{MHz})$ \\
\hline$N_{R B}^{U L}$ & The total nr. of uplink RBs (50) \\
\hline$N_{R B_{D 2 D}}^{U L}$ & The nr. of uplink RBs for Broadcast Group (BG)s (20) \\
\hline$N_{V_{T}}$ & The nr. of D2D BGs \\
\hline$N_{V_{R}}$ & The nr. of receivers per BG \\
\hline$N_{C}$ & The nr. of Cellular User (CU) (10-20) \\
\hline PoolPeriod & The time duration of the resource pool \\
\hline$R_{V_{T}}$ & The transmission range of D2D transmitters $(2 \mathrm{~km})$ \\
\hline$R_{e N B}$ & The coverage range of the eNodeB $(5 \mathrm{~km})$ \\
\hline bitmap & The time resource pattern \\
\hline$T T I$ & Transmission time interval (1 ms) \\
\hline$I_{M C S}$ & Modulation and coding scheme index \\
\hline$M S$ & The size of the message to be transmitted \\
\hline$N_{M S}$ & The number of the messages to be transmitted \\
\hline$L_{C R B}$ & The predefined nr. of contiguous RBs per BG (5) \\
\hline$T B S$ & The predefined transport block size ( 872 bits) \\
\hline$P_{T X}^{D}$ & The D2D transmitter power $(30 \mathrm{dBm})$ \\
\hline$P_{T X}^{C}$ & The CU transmitter power $(40 \mathrm{dBm})$ \\
\hline$P_{R X}^{D}$ & The D2D received signal power $(\mathrm{dBm})$ \\
\hline$S I N R_{T}$ & The tolerable SINR level at a node \\
\hline$N_{0}$ & Noise power spectral density $(-174 \mathrm{dBm} / \mathrm{Hz})$ \\
\hline
\end{tabular}




\subsubsection{Resource allocation}

Based on the 3GPP standardization framework (Release 12), we consider the uplink spectrum for D2D communication and accordingly its physical data channel follows the structure of the PUSCH, using the SC-FDMA technique [22] [20]. The rationale for considering the uplink is three-fold. Compared to downlink, the uplink spectrum is usually underutilized. In uplink, the interference from D2D links may affect the base station which usually has interference cancellation capabilities. While in downlink, it is the cellular users that get involved in interference with D2D users and also the performance of D2D users degrades, due to strong interference from the base station. Further, SC-FDMA has lower peak to average ratio, compared to OFDMA used in downlink and this will result in less energy consumption.

The smallest resource allocation unit that we consider in the model, is a RB. That is, $180 \mathrm{KHz}$ (i.e., $12 \times 15 \mathrm{KHz}$ subcarriers) in frequency and $0.5 \mathrm{~ms}$ (i.e., 1 slot) in time. However, the smallest scheduling time interval in LTE is 2 slots (i.e., 1 subframe), implying the allocation of RBs in pairs. For a given user, the transmission occurs in a set of subframes within the resource pool and in a set of RBs within those subframes. The set of subframes is specified by a bitmap, such that only the subframes with the corresponding status 1 in the bitmap are used for the transmission and from now on we call them "on" subframes / bits [22]. As for the resource blocks, the allocation is based on the Uplink type 0, specified by Resource Indication Value (RIV). RIV is a value specified in the resource allocation field of a scheduling grant message, called Downlink Control Information (DCI). RIV corresponds to two values as the ID of the starting $\mathrm{RB}$ for allocation, denoted by $R B_{\text {start }}$ and a length in terms of the contiguously allocated RBs, denoted by $L_{C R B}$ as presented in Eq. 5.1 [22] (See Figure 5.4). Given RIV, a user is able to figure out the RBs assigned for its transmission.

$$
R I V=\left\{\begin{array}{l}
N_{R B}^{U L}\left(L_{C R B}-1\right)+R B_{\text {start }}, \text { if }\left(L_{C R B}-1\right) \leq \frac{N_{R B}^{U L}}{2} \\
N_{R B}^{U L}\left(N_{R B}^{U L}-L_{C R B}+1\right)+\left(N_{R B}^{U L}-1-R B_{\text {start }}\right), \text { otherwise }
\end{array}\right.
$$

where $N_{R B}^{U L}$ is the total number of uplink RBs.

In LTE the MAC Protocol Data Unit (PDU) transmitted over the Physical channel is called the transport block and its size varies for each user, depending on the value for $L_{C R B}$ and the modulation and coding scheme, specified 
by its index as $I_{M C S}[22]$. For the example case of $L_{C R B}=5$ and $I_{M C S}=10$ (i.e., modulation $16-Q A M$ ), we have a transport block of size 782 bits.

The D2D scheduling interval is PoolPeriod which is the time duration of a resource pool. This implies that every PoolPeriod D2D transmitters obtain new resources, based on the allocation mechanism. The scheduling interval for cellular users is semi-persistent, composed of 8 Transmission Time Interval (TTI)s which is a bitmap length. That is, at each $8 m s$ interval cellular users obtain new resources, scheduled by the base station in an orthogonal manner.

D2D broadcast is an open-loop communication, with no Hybrid Automatic Repeat Query (HARQ) feedback scheme. Hence, transmission repetitions (in four consecutive subframes) are assumed as a means of reliability improvement [22]. That is, the first transport block is transmitted four times in the first four "on" subframes of the resource pool and each next transport block is transmitted four times in the next four "on" subframes. Figure 5.4 shows an example allocation for four broadcast groups within a part of a resource pool with eight subframes (i.e., the length of a bitmap).

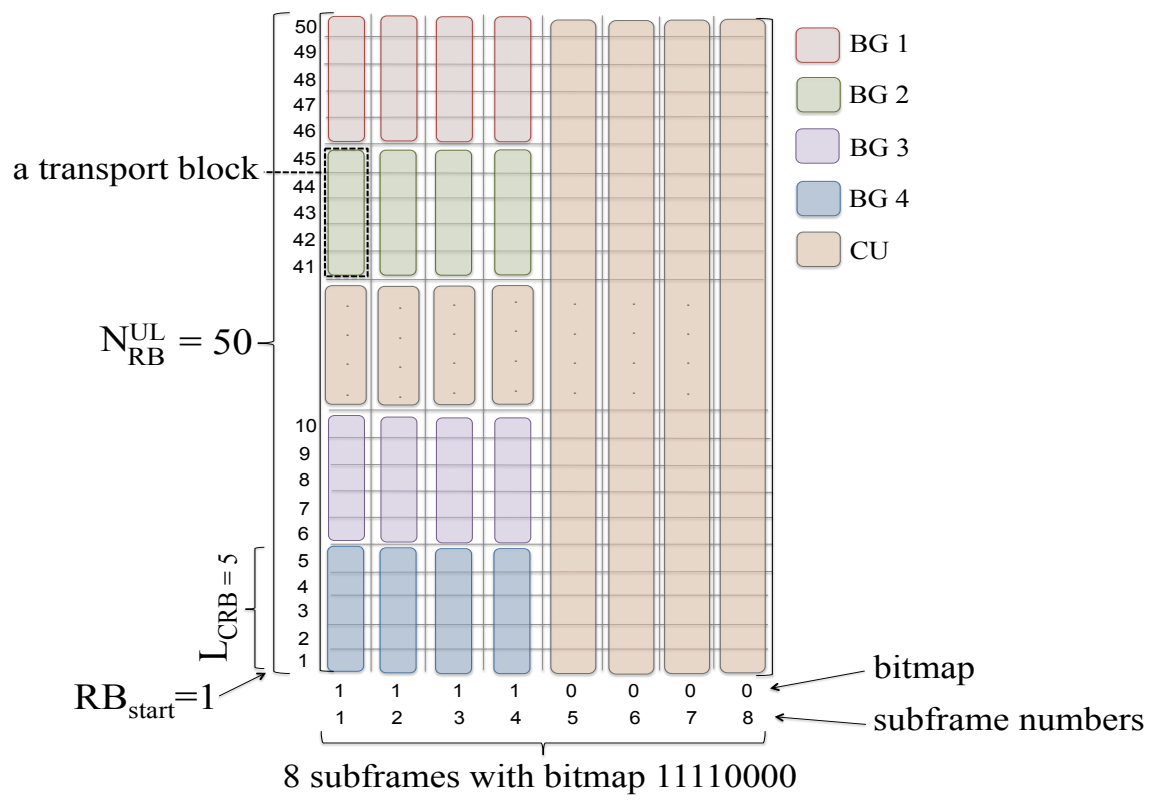

Figure 5.4: An example resource allocation. 


\subsubsection{Propagation model}

The power of the received signal $P_{R X}$ at a given receiver is computed according to

$$
P_{R X}=P_{T X}-L
$$

where $P_{T X}$ and $L$ are the transmission power and the propagation loss in $\mathrm{dB}$, respectively. Many factors may influence the propagation loss $L$, namely path loss, shadowing and fast fading effects due to multipath propagation of the signal. The impact of shadowing on the system performance can be neglected, due to the high transmission power for rather short D2D coverage distance. We also reasonably assume that due to OFDM robustness, the fast fading effect is mitigated. Hence, this is mainly the path loss affecting the signal degradation and for D2D communications, it is modeled using the Hata path loss model [18] $[23]$ as

$$
L=148+40 \log _{10}(d),
$$

where $d$ is the distance between the given transmitter and receiver, in kilometer. With this, $P_{R X}$ can be computed and the SINR determines if this is enough to realize successful reception of data at a given receiver, in the presence of noise and the Interfering Signal (IS)s as

$$
S I N R^{l}=\frac{P_{R X}^{l}}{N^{l}+\sum_{\forall i \in I S} P_{R X_{i}}^{l},}
$$

where $P_{R X_{i}}^{l}$ is the power of the received signal from interferer $i$. Superscript $l$ indicates linear scale.

\subsection{Adaptive resource allocation mechanism}

In this section, we introduce our resource management approach with an adaptive function, driven by change in the network state. The network state is characterized by its density and topology. Our approach aims adapting to the varying network state by means of an efficient and interference-aware resource 
allocation and hence keeping desirable performance. Note that our approach aims D2D resource management within a cell. D2D users of the same cell are the only source of intra-cell interference, since due to considering in-band overlay D2D as mentioned earlier in Section 5.1, the cellular users of the same cell cannot reuse resources reserved for D2D users of the cell. Accordingly, they are not considered in the design of the mechanism. However, as a means of evaluating performance of the mechanism in the presence of inter-cell interference, a second cell composed of both D2D broadcast groups and cellular users is considered, as described later in Section 5.5 and Section 5.6.

We specify the network density as the average channel load level. To quantify this, we define the network parameter load index, denoted by $L I$, as the number of D2D transmitters to be competing for the same resource. From now on, we call them co-transmitters. We assume that the base station has the knowledge about the number of transmitters $\left(N_{V_{T}}\right)$ requesting for D2D broadcast [20], at the beginning of each transmission scheduling interval. Given this and considering all resources as equally probable to be occupied, the ratio of the number of broadcast groups $\left(N_{V_{T}}\right)$ to the available number of resources would be a reasonable approximation of $L I$, known at the base station. For example, for $N_{V_{T}}=8$ and the number of available resources $N_{R B_{D 2 D}}^{U L} / L_{C R B}=20 / 5=4$, we have $L I=8 / 4=2$.

We also define an upper limit for $L I$, denoted by $L I_{T}$, as the maximum tolerable channel load (i.e., the maximum number of co-transmitters per channel) to avoid interference. Considering the area of a given cell with radius $R_{e N B}$ and the transmission range $R_{V_{T}}$ of broadcast groups, $L I_{T}$ can be characterized as the maximum number of co-transmitters in the cell with pairwise distance no less than $2 \times R_{V_{T}}$, in order to avoid interfering co-transmissions, as demonstrated in the example Figure 5.5. This can be simplified and dealt with as a circle packing problem. Though, for vehicles residing exactly on the border of the transmission range $R_{V_{T}}$ of the corresponding transmitter, the desired signal can be of the same strength of interfering signals from other transmitters. $L I_{T}$ is reasonably approximated by $L I_{T}=\left\lfloor 0.83 \times\left(R_{e N B} / R_{V_{T}}\right)^{2}-1.9\right\rfloor[92][62]$.

As demonstrated in Algorithm 1, the base station acquires $L I$ and $L I_{T}$ at the beginning of each transmission scheduling interval (i.e., PoolPeriod) and takes the appropriate action accordingly, based on the network density (specified by three possibilities for the traffic regime $\left(L I \leq 1,1<L I \leq L I_{T}, L I>L I_{T}\right)$ and the network topology which we explain in what follows.

$\mathbf{L I} \leq \mathbf{1}$. Such a traffic regime implies that the number of D2D transmitters, seeking for resources, is less than the number of available resources $R$. In such a case there is no need for resource reuse and the base station schedules the resources for D2D transmitters, in an orthogonal way. Hence, there would be one-to-one correspondence between each user and a resource. 


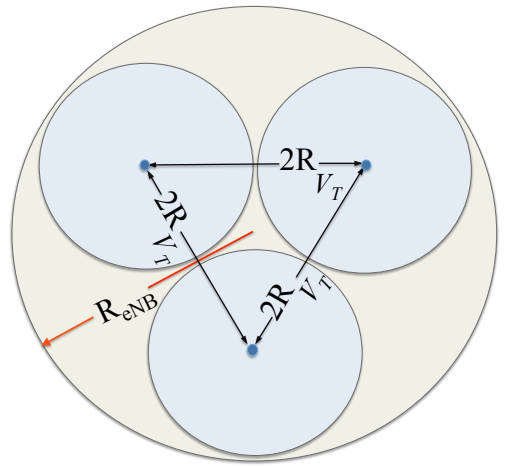

Figure 5.5: Three non-overlapping D2D transmitters within a cell.

$\mathbf{1}<\mathbf{L I} \leq \mathbf{L I}_{\mathbf{T}}$. In this case, resource reuse is inevitable as the number of D2D transmitters is more than the number of available resources $R$. Since $L I$ is less than the upper limit, presumably the users can be served interference-free with the current number of available resources, based on which $L I$ is computed. This would be checked by the base station as follows. Given the availability of users' location information at the base station, the adjacency graph of the current D2D transmitters is obtained. Such that, each D2D transmitter represents a vertex in the graph and there exist edges between every two D2D vertices, with distance less than $2 \times R_{V_{T}}$. Given this, the base station separates all vertices into as few as possible disjoint maximal independent subsets. The D2D transmitters represented by corresponding vertices in each subset are supposed to use an identical resource, since they are pairwise non-adjacent and we call them a reuse group/subset. If the number of these reuse groups is less than or equal to the number of radio resources $R$, there would be one-to-one correspondence between each reuse group/subset and a resource. That is, all users belonging to a reuse group/subset can safely reuse their assigned resource, without experiencing strong interference. We consider all the resources equally probable to be assigned to a reuse group/subset. Note that as a means of resource efficiency, the algorithm tries to keep the number of the reuse groups as few as possible. We call this approach spatial resource reuse.

It can also happen that due to topology limitation, it is not feasible to achieve the grouping of users into $R$ or fewer number of disjoint subsets of independent vertices. In such a circumstance, the approach specified for the case $L I>L I_{T}$ is adopted.

$\mathbf{L I}>\mathbf{L I}_{\mathbf{T}}$. In this case $L I$ exceeds its upper limit and serving the users with the current $L_{C R B}$ may lead to significant performance degradation, due to the 
increasing number of interfering users. Depending on the application requirement, either of the following procedures would be adopted. The first option is using the spatial resource reuse approach described for the case $1<L I \leq L I_{T}$ by keeping the number of resources unchanged. Given this, the users are grouped into $R$ disjoint subsets which are no longer independent. This implies that some users will inevitably experience interference. However, in order to keep the number of these users as low as possible, the subsets are created with the minimum possible number of edges, as clarified in the algorithm. Once formed, each of these $R$ subsets would be coupled with a resource. According to the second option, fewer RBs are considered for allocation to each user. This gives room to obtain a higher number of resources out of total $N_{R B_{D 2 D}}^{U L}$ number of RBs. To achieve this, $L I$ is set equal to its upper limit $L I_{T}$ and the new number of resources $R$ would be extracted from this equation. Subsequently, the new $L_{C R B}$ (i.e., number of RBs per user) is derived. Accordingly, the reuse groups/subsets would be formed and associated to the new number of resources, as explained earlier for the case $L I<L I_{T}$. Note that due to the reduced number of RBs, the transmission of the message(s) may not be fulfilled within one PoolPeriod. In such a circumstance, two alternatives are considered. According to the first one, the transmission would be extended to the next PoolPeriod(s). We call this alternative spatial resource reuse with data rate adjustment, since transmission of the same amount of data over a longer period of time leads to a lower data rate. According to the second alternative, a higher order modulation would be applied, in order to fulfill the transmission of the message(s) within one PoolPeriod. We call this alternative spatial resource reuse with adaptive modulation.

Figure 5.6 shows the proposed adaptive resource allocation mechanism, as explained above, via a block diagram. As clear from the figure, different choices are to be adopted, according to the traffic regime characterized by the network density and spatial distribution of vehicles characterized by the network topology. As mentioned earlier, in case of the traffic regime $1<L I<=L I_{T}$, it is mostly feasible the grouping of the network nodes into $R$ number of maximimal independent subsets. It means that we only rely on the "spatial resource reuse" aspect of the proposed resource allocation mechanism. If due to topology constraints such grouping is not feasible (and also in case of the system overload $\left(L I>L I_{T}\right)$ ), the number of RBs to be allocated per user can be reduced or not (in such a case, the subsets are no longer independent from each other).

We differentiate between two modeling approaches, where in the first one we consider both reducing (i.e., considering "spatial resource reuse + reduced $n r$. of $R B s "$ ) and not reducing (i.e., considering only "spatial resource reuse") the number of RBs to be allocated per user. In order to compensate the resource shortage in case of the reduced number of $\mathrm{RBs}$, data rate adjustment is considered. Such an approach can be considered 
for applications with no strict latency requirement. In the second modeling approach, the number of RBs to be allocated per user is definitely reduced (i.e., considering "spatial resource reuse + reduced $n r$. of RBs") in case of necessity and in order to compensate the resource shortage adaptive modulation is considered at the expense of higher error rates. This approach is suitable for highly latency-sensitive applications. The two modeling approaches are discussed in detail in Section 5.5 and Section 5.6, respectively.

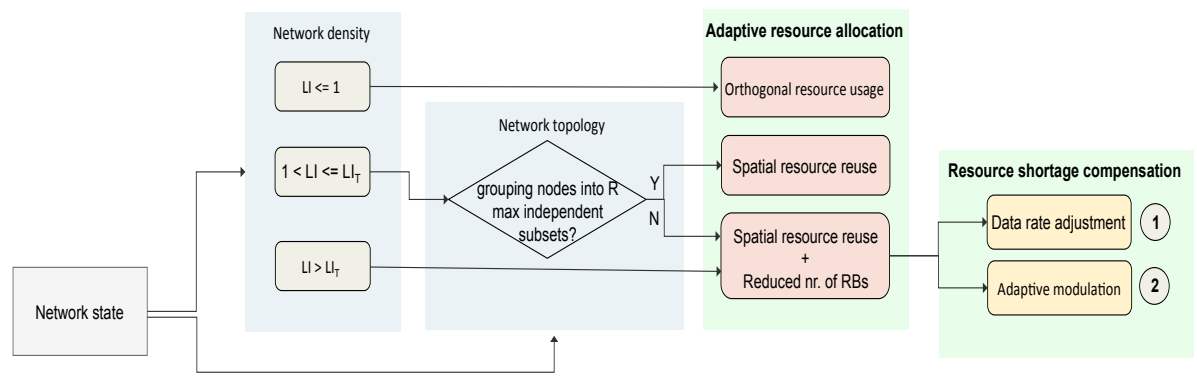

Figure 5.6: Adaptive resource allocation mechanism functional block diagram. 
Algorithm 1 Adaptive resource allocation.

Input: $N_{V_{T}}, B W, L_{C R B}, N_{R B_{D 2 D}}^{U L}, R_{V_{T}}, R_{e N B}$

Output: Load and location aware "BG-resource" coupling initialization:

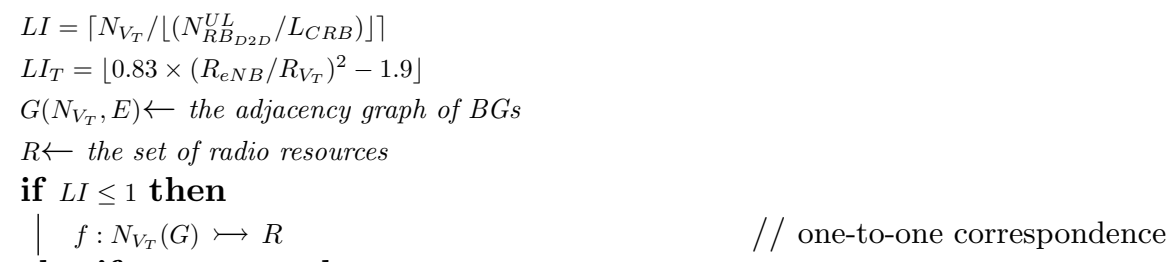

1 else if $1<L I \leq L I_{T}$ then

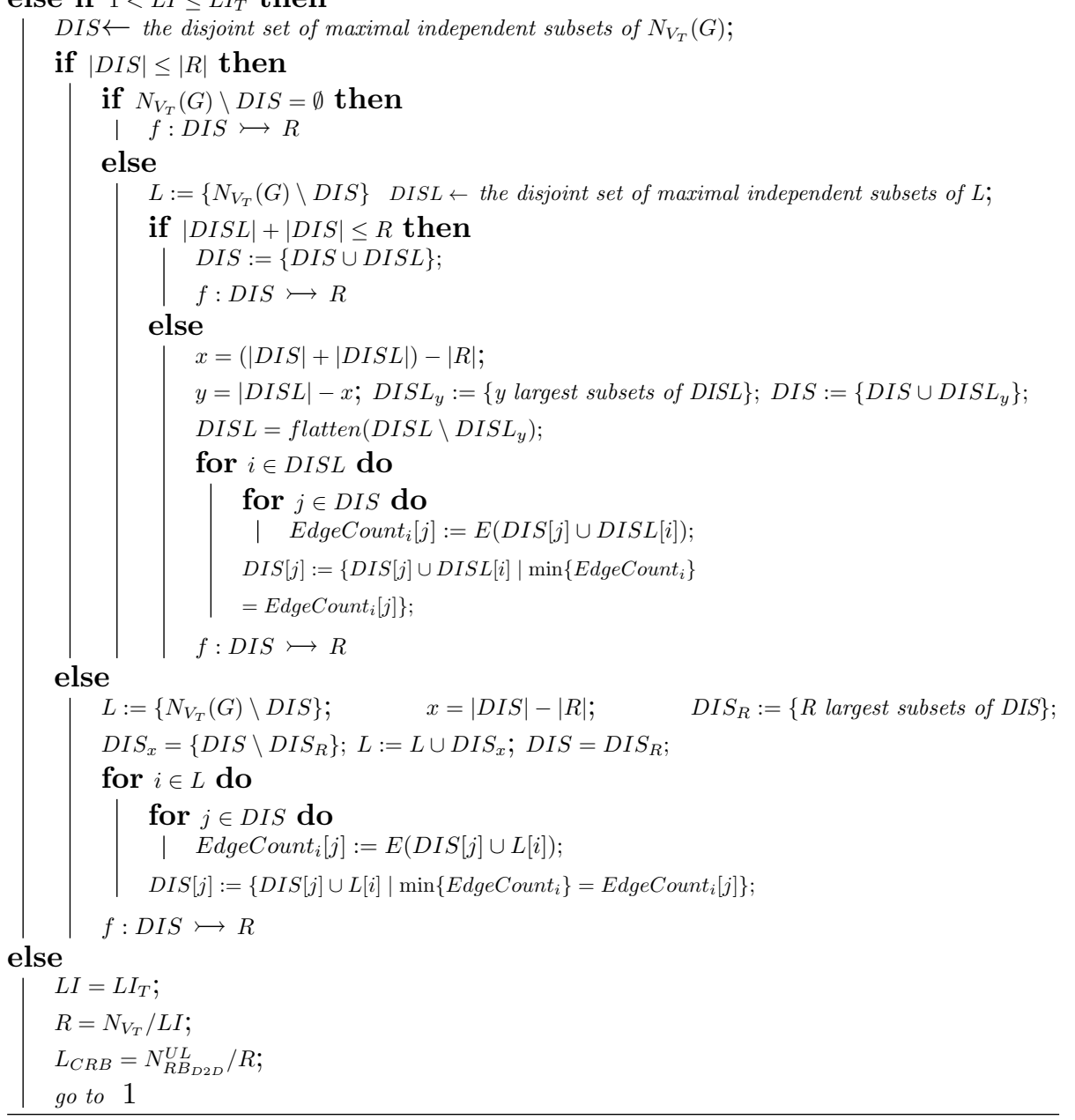




\subsection{Modeling approach 1}

In this section, we present the first modeling approach which we consider for evaluating the performance of the resource allocation mechanism proposed in Section 5.4.

In this approach, we consider a situation where a given number of vehicles in various locations in a cell, get active at the same time as D2D transmitters to successively broadcast messages of the same type to the vehicles around, given the application-driven transmission range. Hence, we are in a situation where there are multiple vehicular users demanding for instantaneous and concurrent broadcast of information to their neighborhood, using D2D links enabled by the LTE wireless communication interface. Hence, they seek for radio resources and upon access, ensuing the allocation mechanism, they broadcast messages to all the intended receivers within their coverage. We call D2D transmitters along with the vehicles in their coverage a BG. What we are interested in, is to evaluate the performance of the applications demanding such instantaneous transmission opportunities, given the proposed resource allocation mechanism.

Table 5.2 lists for each cell the parameters and the corresponding values, unless otherwise mentioned later.

Table 5.2: Parameter values for modeling approach 1.

\begin{tabular}{|c|c|}
\hline Parameter & Value \\
\hline \hline$N_{V_{T}}$ & $1-20$ \\
\hline$N_{V_{R}}$ & 10 \\
\hline PoolPeriod & $40-120 \mathrm{~ms}$ \\
\hline$I_{M C S}$ & 10 \\
\hline$M S$ & $5-15$ Packets \\
\hline$N_{M S}$ & 100 \\
\hline$S I N R_{T}$ & $10 \mathrm{~dB}$ \\
\hline
\end{tabular}

The formation of multiple broadcast groups in a cell may turn the system capacity into a bottleneck given the load incurred over the course of broadcasting messages by those groups. Recalling the functionality of the proposed adaptive resource allocation mechanism introduced in Section 5.4 (See Figure 5.6), under heavy loads of high number of BGs (i.e., $L I>L I_{T}$ ) the number of RBs allocated per user can be decreased (i.e., relying on spatial resource reuse and reduced 
number of RBs) or not (i.e., relying only on spatial resource reuse). In this modeling approach, we consider both options for performance evaluation and this is indicated while presenting the results. The alternative in order to cope with the resource shortage in case of the reduced number of RBs, is "data rate adjustment". That is, broadcast of multiple messages unachievable within duration of one PoolPeriod, is to be accomplished over consecutive PoolPeriods and as a result of this data rate is reduced.

Figure 5.7 shows an example modeling scenario, with the left-side cell being of our focus for performance evaluation and the other cell considered as a means of inter-cell interference impact on broadcast performance. For this, the following settings are applied. We consider $N_{V_{T}}$ D2D transmitters distributed following uniformly random positions in the area of a cell with one base station, each of them having $N_{V_{R}}$ receivers, also with uniformly distributed random positions within the coverage $R_{V_{T}}$ of the transmitter. Note that, due to the overlay resource allocation scheme in this work, the cellular users do not have any interference effect on D2D broadcast groups of the same cell and hence they are not considered in the main cell for performance evaluation. Given this, the intra-cell interference would be due to the resource reuse between broadcast groups in the cell. As a means of considering the interference also at the inter-cell level, we consider a second cell with the same assumptions, except the fact that we also consider cellular users with uniformly distributed random positions. Therefore, the inter-cell interference can be originated by cellular users and/or broadcast groups in the second cell, reusing resources of broadcast groups in the main cell.

Note that in case of overlapping broadcast neighborhoods, receiver vehicles are associated only with one of the broadcaster vehicles. Also, we consider half-duplex D2D communication, so that a broadcaster is not considered as the receiver of another broadcaster.

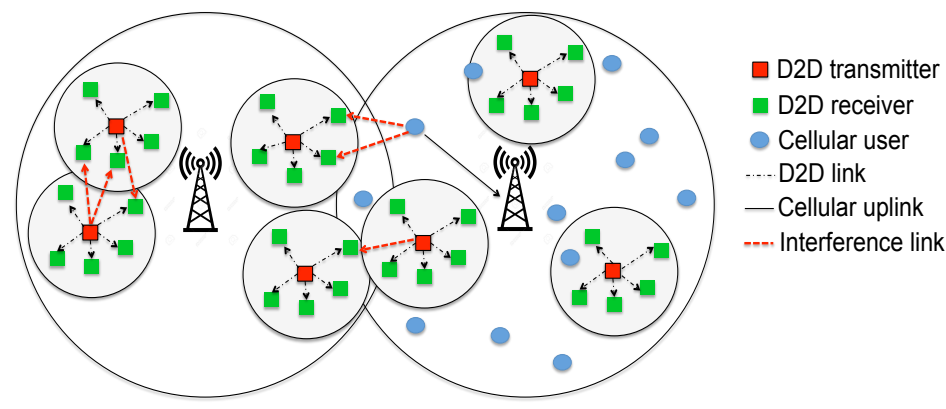

Figure 5.7: An example modeling scenario. 
In this model we define $S I N R_{T}$, as the threshold $S I N R$ required for coverage. Accordingly, transmission to an arbitrary receiver is considered successful or failed, given the $S I N R$ of the signal received by the receiver being higher or lower than this threshold, respectively.

\subsubsection{Performance analysis}

In this section, for the considered modeling approach we first introduce the performance measures and accordingly present the obtained results. For this, we implement the proposed model and resource allocation mechanism in Matlab and then by considering key parameters as inputs to the system, evaluate its performance in terms of the introduced metrics.

\subsubsection{Evaluation metrics}

Complete Message Delivery Ratio (CMDR) Considering the reliability requirement of D2D broadcasting, in particular for safety-critical vehicular applications, the complete message delivery ratio is a relevant metric, representing the average percentage of receivers of an arbitrary broadcast group that successfully receive "all" the broadcast messages, as shown in Eq. 5.5.

$$
C M D R=\frac{\sum_{i=1}^{N_{V_{T}}} \frac{N_{V_{R}}^{i}}{N_{V_{R}}}}{N_{V_{T}}} \times 100,
$$

where $N_{V_{R}}^{i}$ is the number of receivers of the broadcast group $i$ that successfully receive all the messages.

Spectrum Efficiency (SE) Due to the rapidly growing demand, efficient utilization of the scarce spectrum is of significant importance, mainly from the system point of view. This metric specifies how efficiently the available radio resources are utilized by indicating the success ratio per unit bandwidth and is defined according to Eq. 5.6.

$$
S E=\frac{N_{V_{T}} \times(C M D R / 100)}{\# R B s},
$$


where \#RBs refers to the number of utilized RBs out of the ones $\left(N_{R B_{D 2 D}}^{U L}\right)$ available for D2D resource allocation.

Packet Loss Ratio (PLR) Most applications demand timely delivery of data. To assure this timeliness transmissions are considered over a limited range of the time domain of the spectrum (specified via PoolPeriod by the application) and it is indicated in terms of the packet loss ratio if this timeliness is met. For an arbitrary receiver, this metric refers to the ratio between the lost packets (i.e., transport blocks) and the total number of broadcast packets, as shown in Eq. 5.7.

$$
P L R=\frac{\sum_{i=1}^{N_{V_{T}}} \frac{\sum_{j=1}^{N_{V_{R}}} \frac{(\text { \#lost packets })^{i, j}}{N_{M S} \times M S}}{N_{V_{R}}}}{N_{V_{T}}} \times 100,
$$

where (\#lost packets) $)^{i, j}$ is the number of lost packets of receiver $j$ of broadcast group $i$.

\subsubsection{Numerical results}

The performance of the system, evaluated in terms of the above-mentioned metrics, is the focus of this section. Considering effective factors such as the number of broadcast groups $N_{V_{T}}$, the message size $M S$, the time duration of the resource pool PoolPeriod as inputs to the system, we analyze the system functionality. Note that being within $95 \%$ confidence intervals, the results for each numerical setting are obtained by averaging over twenty random topologies of a given number of broadcast groups, uniformly distributed in the area of the main cell. We consider the the random reuse resource allocation approach, as the baseline for comparison to our proposed approach. As the name implies, in this approach radio resources are chosen on a random basis from the set of resources available in the D2D resource pool. The results help providing insight on how the system performs under different assumptions.

The complete message delivery ratio for an arbitrary receiver, against increasing density of D2D broadcast groups is shown in Figure 5.8 for the proposed adaptive approach and the random reuse approach, as the baseline for comparison. Note that in this figure both the single cell and two cell cases are considered. With a single cell in isolation, it is better seen the improvement 
provided by the proposed approach, independent of the inter-cell interference in a multicell scenario. Also, in this figure the spatial resource reuse approach is considered with no data rate adjustment in order to better see the impact of spatial resource reuse, distinctly. What is first observed in this figure, is that even without data rate adjustment in case of dense scenarios, the proposed approach succeeds in keeping the delivery ratio significantly higher than the baseline approach.

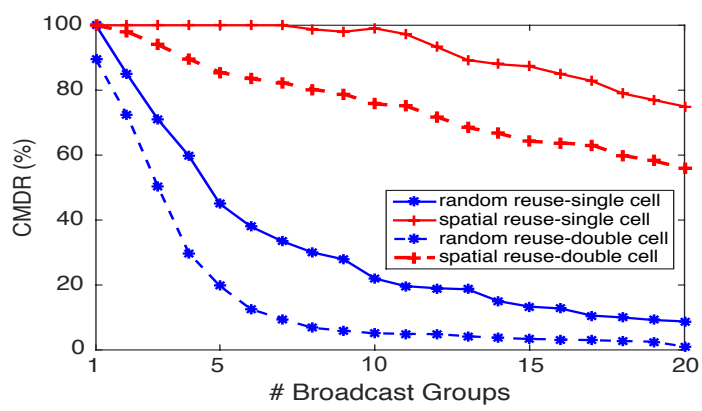

Figure 5.8: CMDR PoolPeriod=40ms, MS=1500Byte.

Note that in the setting for the two cell scenario, a uniformly distributed random number of broadcast groups between 1 and 10 are considered in the second cell, with the baseline resource allocation approach. Despite the corruptive effect of the inter-cell interference on the delivery ratio, compared to the single cell case, it is recognized a significant improvement against the random reuse scenario.

Figure 5.9 shows the spectrum efficiency against increasing number of broadcast groups, for the same setting.

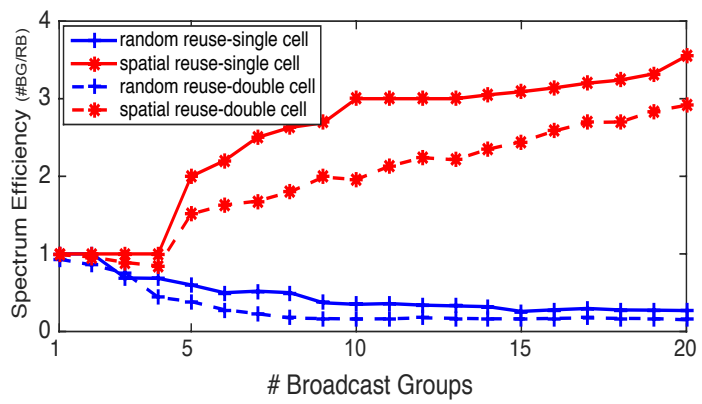

Figure 5.9: Spectrum efficiency, PoolPeriod $=40 \mathrm{~ms}, \mathrm{MS}=1500$ Byte 
Despite increasing density of D2D broadcast groups in the cell, the radio resources are used in a more conservative way via the proposed approach, due to the purposive spatial reuse. Whereas, the random reusing of the resources comes significantly short of successfully serving denser scenarios. Due to the one-to-one correspondence between each user and a resource in our approach, the spectrum efficiency equals the delivery ratio up to the first four broadcast groups. After this point, due to the spatial reuse of the resources, the number of utilized resources is less than the number of served broadcast groups. Recalling from Eq. 5.6, this results in the significant increase in the spectrum efficiency, as traceable in the figure. Though by taking the effect of inter-cell interference into account, the resource efficiency is shrinked compared to the single cell case, we still see a significant gap between two curves. Note that the resource allocation in the second cell follows the baseline approach.

The average packet loss ratio for a receiver of an arbitrary broadcast group is shown in Figure 5.10, against the increasing density of D2D broadcast groups, for the proposed adaptive approach and the random one. While the random approach deteriorates quickly towards denser scenarios, our approach performs noticeably more reliable and scalable.

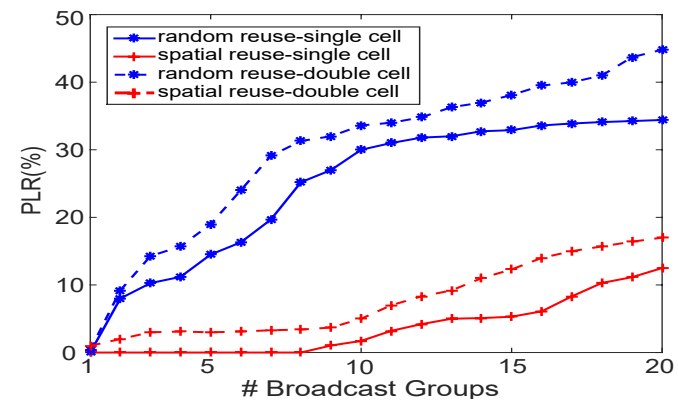

Figure 5.10: PLR, PoolPeriod=40ms, MS=1500Byte.

The complete message delivery ratio against increasing number of broadcast groups and the message size is demonstrated in Figure 5.11 for a two cell scenario. Besides the decreasing flow of the delivery ratio in both directions as expected, it is clearly visible that our approach distinctly outperforms the baseline approach. Again with significant difference between two approaches, Figure 5.12 shows the packet loss ratio for the same setting.

In Figure 5.13 we can see the effect of the data rate adjustment on the delivery ratio, in dense scenarios. Note that here the data rate adjustment is applied only for the cases where $L I>L I_{T}$. That is, for the number of broadcast groups more than 13 , given $L I_{T}=\left\lfloor 0.83 \times(5 / 2)^{2}-1.9\right\rfloor=3$. The improvement of 


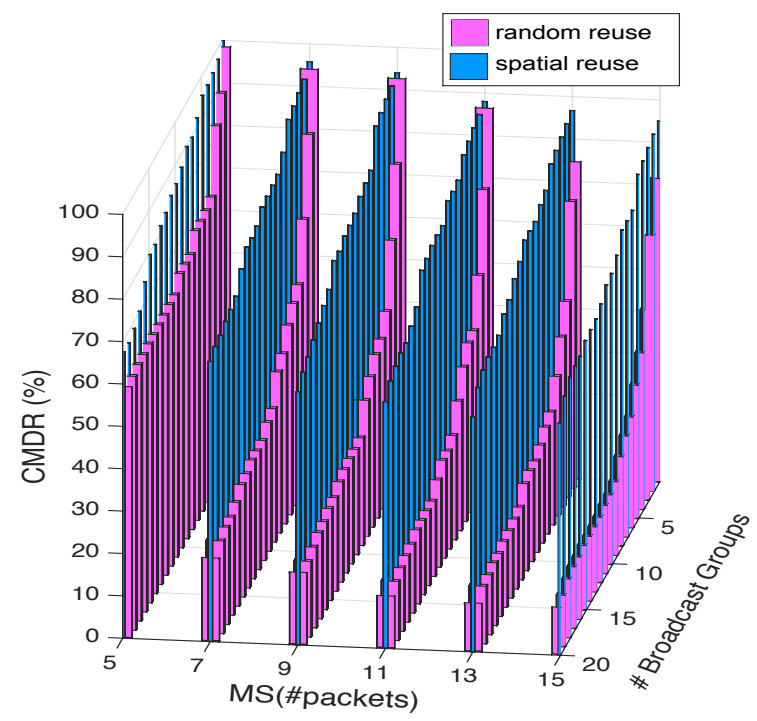

Figure 5.11: CMDR, PoolPeriod=100ms.

the delivery ratio is achieved at the expense of lower data rates as shown in the table corresponding to Figure 5.13. As mentioned earlier, the criteria for data rate adjustment is derived by the application requirements on the data rate and delivery ratio. Hence, it could be adopted a system either serving an increasing number of broadcast groups and decreasing the data rate within the allowed range (if needed) or blocking the new broadcast groups, in order to keep the data rate unchanged. In this figure, we consider the first case and with the maximum number of 20 broadcast groups, the data rate is not less than the minimum we considered (i.e., $0.328 \mathrm{Mbps}$, corresponding to $2 \mathrm{RBs}$ ). 


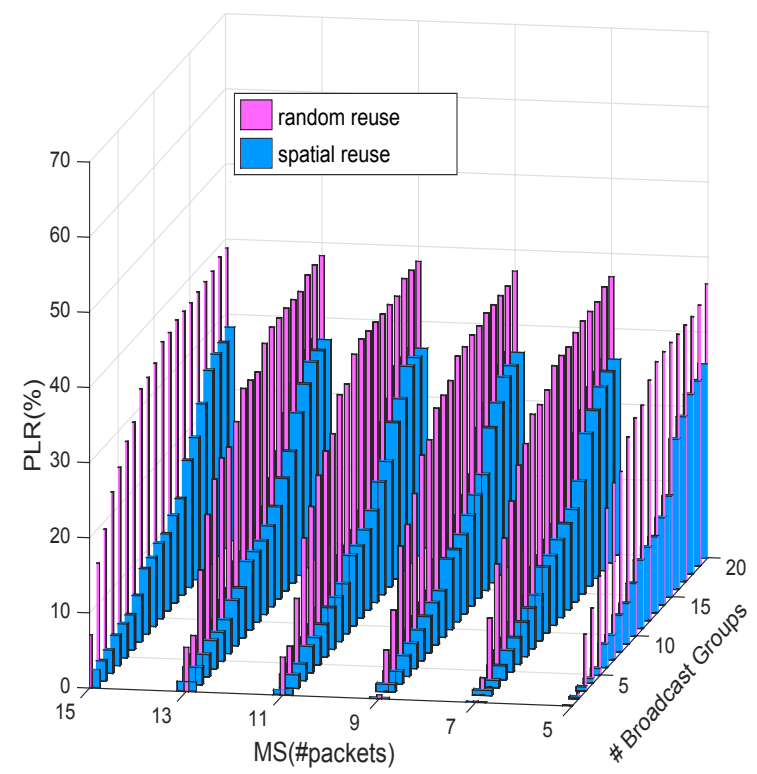

Figure 5.12: PLR, PoolPeriod=100ms.

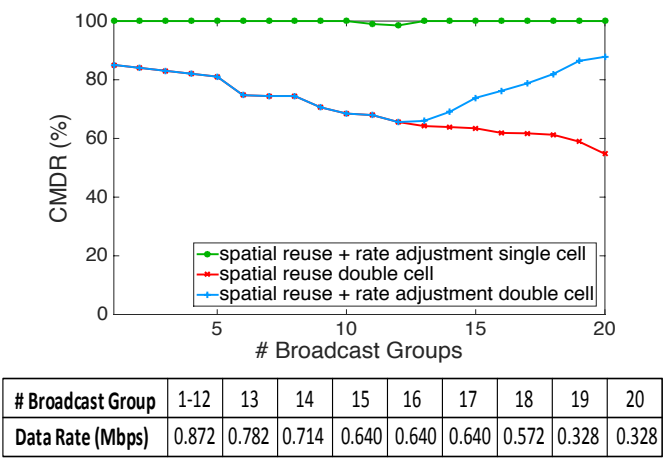

Figure 5.13: CMDR, PoolPeriod=100ms, MS=1500Byte.

\subsection{Modeling approach 2}

In this section, we present the second modeling approach, where we consider for evaluating the performance of our adaptive resource allocation mechanism proposed in Section 5.4. As one may recall, in the first modeling approach, 
we considered a constant number of concurrently active broadcast groups and investigated how they perform while completing broadcast of $N_{M S}$ number of messages. Under high loads, in that approach transmissions were carried out with lower data rates and over longer periods of consecutive PoolPeriods, due to fewer number of RBs allocated per user.

The second modeling approach differs from the first one with respect to three aspects. Firstly, we consider a varying number of active broadcast groups. That is, an arbitrary number of vehicles, drawn from the Poisson distribution with rate $\lambda$ (we call it BG activation rate), get active (as a result of emergency situations occurred within the cell) among a given high number of vehicles with uniformly random positions, at the beginning of a transmission scheduling interval (i.e., a PoolPeriod). Second, in this modeling approach, broadcast groups are supposed to fulfill broadcast of a single message within duration of a PoolPeriod. This is of relevance to safety-critical applications where such a situation may arise in the wake of an emergency and reliable broadcast within short duration of time is of high importance. And third, the number of receivers per broadcast group is not constant, and vehicles falling within the application-driven coverage range of an active vehicle are considered as its intended receivers and accordingly a broadcast group is formed.

Recalling the functionality of the proposed adaptive resource allocation mechanism introduced in Section 5.4 (See Figure 5.6), under heavy loads of high number of BGs (i.e., $L I>L I_{T}$ ) the number of RBs allocated per user can be decreased (i.e., relying on spatial resource reuse and reduced number of $\mathrm{RBs}$ ) or not (i.e., relying only on spatial resource reuse). In this modeling approach 2, we consider the first case for performance evaluation. In order to cope with the resource shortage in case of the reduced number of RBs, the "adaptive modulation" alternative is considered. That is, a higher order modulation scheme is adopted (i.e., more bits of information are carried per modulation symbol), in order to fit data and accordingly accomplish the transmission of all BGs within the duration of one PoolPeriod. One may note that this comes at the expense of higher error probabilities, in particular for receiver vehicles farther away from the transmitter. Hence, this translates to shorter ranges of successful transmission.

In what follows, we describe the process, by first elaborating on how to obtain the relevant Transport Block Size (TBS). For a Transport Block (TB) that is not mapped to more than single-layer spatial multiplexing (i.e., Single Input Single Output (SISO)), the TBS is determined by the procedure in Subclause 7.1.7.2.1 of [22]. For this, we refer to Table 7.1.7.2.1-1: "Transport block size table" [22] (See Figure 5.14), where its $\left(I_{T B S}, N_{P R B}\right)^{t h}$ entry represents the TBS (in terms of the number of bits of data that it can carry) composed of $N_{P R B}$ number of RBs and characterized by its relevant index $I_{T B S}$. Now, given 
the new number of RBs allocated per user as a TB (calculated as a result of the adaptive resource allocation mechanism proposed in Section 5.4) and the message size $M S$, the highest possible TBS is chosen from Table 7.1.7.2.1-1 such that message transmission can be completed within duration of a PoolPeriod. Subsequently, the modulation scheme is determined from Table 8.6.1-2 in [22] (See Figure 5.15) by referring to the $I_{M C S}$ corresponding to the $I_{T B S}$, obtained from Table 7.1.7.2.1-1.

\begin{tabular}{|c||c|c|c|c|c|c|c|c|c|c|}
\hline \multicolumn{1}{|c||}{} & \multicolumn{10}{c|}{$N_{\text {TBS }}$} \\
\cline { 2 - 12 } & $\mathbf{1}$ & $\mathbf{2}$ & $\mathbf{3}$ & $\mathbf{4}$ & $\mathbf{5}$ & $\mathbf{6}$ & $\mathbf{7}$ & $\mathbf{8}$ & $\mathbf{9}$ & $\mathbf{1 0}$ \\
\hline \hline 0 & 16 & 32 & 56 & 88 & 120 & 152 & 176 & 208 & 224 & 256 \\
\hline 1 & 24 & 56 & 88 & 144 & 176 & 208 & 224 & 256 & 328 & 344 \\
\hline 2 & 32 & 72 & 144 & 176 & 208 & 256 & 296 & 328 & 376 & 424 \\
\hline 3 & 40 & 104 & 176 & 208 & 256 & 328 & 392 & 440 & 504 & 568 \\
\hline 4 & 56 & 120 & 208 & 256 & 328 & 408 & 488 & 552 & 632 & 696 \\
\hline 5 & 72 & 144 & 224 & 328 & 424 & 504 & 600 & 680 & 776 & 872 \\
\hline 6 & 328 & 176 & 256 & 392 & 504 & 600 & 712 & 808 & 936 & 1032 \\
\hline 7 & 104 & 224 & 328 & 472 & 584 & 712 & 840 & 968 & 1096 & 1224 \\
\hline 8 & 120 & 256 & 392 & 536 & 680 & 808 & 968 & 1096 & 1256 & 1384 \\
\hline 9 & 136 & 296 & 456 & 616 & 776 & 936 & 1096 & 1256 & 1416 & 1544 \\
\hline 10 & 144 & 328 & 504 & 680 & 872 & 1032 & 1224 & 1384 & 1544 & 1736 \\
\hline 11 & 176 & 376 & 584 & 776 & 1000 & 1192 & 1384 & 1608 & 1800 & 2024 \\
\hline 12 & 208 & 440 & 680 & 904 & 1128 & 1352 & 1608 & 1800 & 2024 & 2280 \\
\hline 13 & 224 & 488 & 744 & 1000 & 1256 & 1544 & 1800 & 2024 & 2280 & 2536 \\
\hline 14 & 256 & 552 & 840 & 1128 & 1416 & 1736 & 1992 & 2280 & 2600 & 2856 \\
\hline 15 & 280 & 600 & 904 & 1224 & 1544 & 1800 & 2152 & 2472 & 2728 & 3112 \\
\hline 16 & 328 & 632 & 968 & 1288 & 1608 & 1928 & 2280 & 2600 & 2984 & 3240 \\
\hline 17 & 336 & 696 & 1064 & 1416 & 1800 & 2152 & 2536 & 2856 & 3240 & 3624 \\
\hline 18 & 376 & 776 & 1160 & 1544 & 1992 & 2344 & 2792 & 3112 & 3624 & 4008 \\
\hline 19 & 408 & 840 & 1288 & 1736 & 2152 & 2600 & 2984 & 3496 & 3880 & 4264 \\
\hline 20 & 440 & 904 & 1384 & 1864 & 2344 & 2792 & 3240 & 3752 & 4136 & 4584 \\
\hline 21 & 488 & 1000 & 1480 & 1992 & 2472 & 2984 & 3496 & 4008 & 4584 & 4968 \\
\hline 22 & 520 & 1064 & 1608 & 2152 & 2664 & 3240 & 3752 & 4264 & 4776 & 5352 \\
\hline 23 & 552 & 1128 & 1736 & 2280 & 2856 & 3496 & 4008 & 4584 & 5160 & 5736 \\
\hline 24 & 584 & 1192 & 1800 & 2408 & 2984 & 3624 & 4264 & 4968 & 5544 & 5992 \\
\hline 25 & 616 & 1256 & 1864 & 2536 & 3112 & 3752 & 4392 & 5160 & 5736 & 6200 \\
\hline 26 & 712 & 1480 & 2216 & 2984 & 3752 & 4392 & 5160 & 5992 & 6712 & 7480 \\
\hline $26 A$ & 632 & 1288 & 1928 & 2600 & 3240 & 3880 & 4584 & 5160 & 5992 & 6456 \\
\hline & & & & & & & & & & \\
\hline
\end{tabular}

Figure 5.14: Part of the "Transport block size table (dimension $34 \times 110)$ " [22].

\subsubsection{Performance analysis}

In this section, for the considered modeling approach we first introduce the performance measures and accordingly present the obtained results. For this, we implement the proposed model and resource management scheme in Matlab and then by considering key parameters as inputs to the system, evaluate its performance in terms of the introduced metrics. 


\begin{tabular}{|c||c|c|}
\hline $\begin{array}{c}\text { MCS Index } \\
I_{\mathrm{MCS}}\end{array}$ & $\begin{array}{c}\text { Modulation Order } \\
Q_{\boldsymbol{m}}\end{array}$ & $\begin{array}{c}\text { TBS Index } \\
I_{\mathrm{TBS}}\end{array}$ \\
\hline $\mathbf{0}$ & 2 & 0 \\
\hline $\mathbf{1}$ & 2 & 1 \\
\hline $\mathbf{2}$ & 2 & 2 \\
\hline $\mathbf{3}$ & 2 & 3 \\
\hline $\mathbf{4}$ & 2 & 4 \\
\hline $\mathbf{5}$ & 2 & 5 \\
\hline $\mathbf{6}$ & 2 & 6 \\
\hline $\mathbf{7}$ & 2 & 7 \\
\hline $\mathbf{8}$ & 2 & 8 \\
\hline $\mathbf{9}$ & 2 & 9 \\
\hline $\mathbf{1 0}$ & 2 & 10 \\
\hline $\mathbf{1 1}$ & 4 & 10 \\
\hline $\mathbf{1 2}$ & 4 & 11 \\
\hline $\mathbf{1 3}$ & 4 & 12 \\
\hline $\mathbf{1 4}$ & 4 & 13 \\
\hline $\mathbf{1 5}$ & 4 & 14 \\
\hline
\end{tabular}

Figure 5.15: "Modulation and TBS index table for PUSCH table" [22].

Table 5.3: Parameter values for modeling approach 2.

\begin{tabular}{|c|c|}
\hline Parameter & Value \\
\hline \hline$\lambda$ (the Poisson arrival rate of D2D BGs) & $1-30$ \\
\hline PoolPeriod & $120 \mathrm{~ms}$ \\
\hline$N_{M S}$ & 1 \\
\hline
\end{tabular}

\subsubsection{Evaluation metrics}

Message Error Ratio (MER) Reliability is a performance measure of our focus and a requirement of practically all application types, relying on data transmission, with the safety critical vehicular applications being in the lead. In order to quantify that in our case, we define MER as the probability for a broadcast message not to be received correctly at the receiver side and in what follows we explain how to obtain it.

Figure 5.16 demonstrates the hierarchical levels of data built up into a message that we consider in this work and as one may note MER depends on error ratio of its building components as BitErrorRatio(BER), BlockErrorRatio(BLER) and SegmentErrorRatio(SER) which are assumed to be Independent and Iden- 
tically Distributed (IID). In what follows, we detail on steps (mapped to each level presented in Figure 5.16) taken in order to obtain MER.

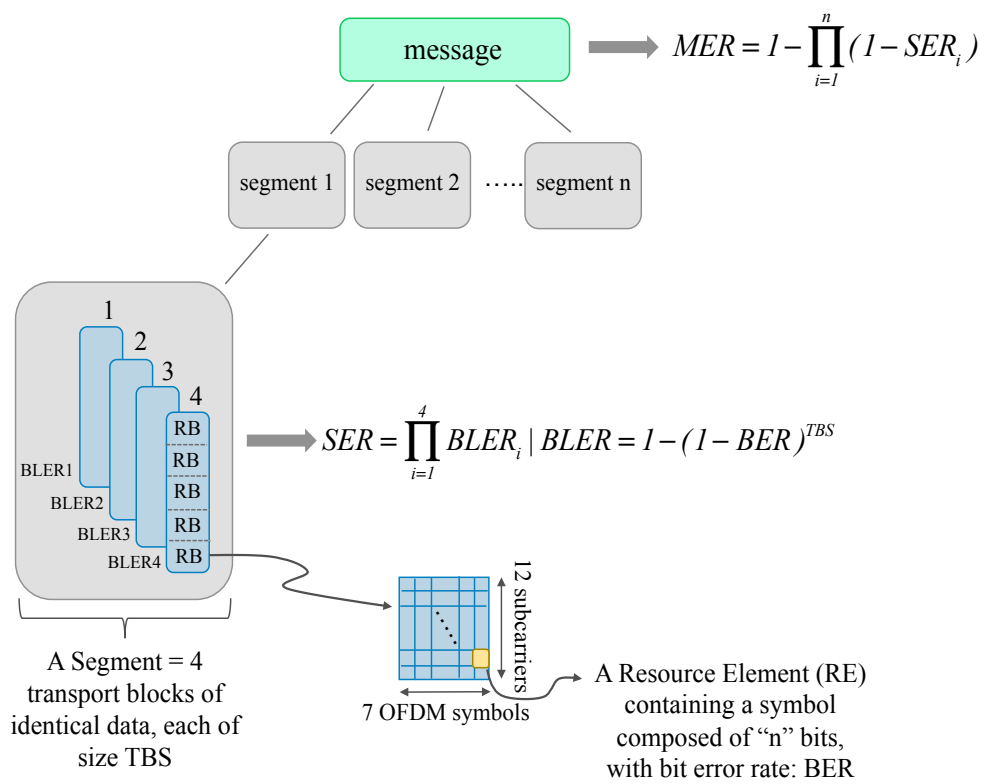

Figure 5.16: The hierarchical structure of a message.

The $B E R$ depends on the $S I N R$ per bit [32] (i.e., $E_{b} / N_{0}$ ) and the ability of the utilized modulation scheme in decoding the bit with that level of $E_{b} / N_{0}$. For square $M-Q A M$, with even $k=\log _{2} M$ (considered in this work), it is given by the following equation [42] [155]:

$$
\begin{aligned}
B E R=\frac{2}{\sqrt{M} \log _{2} \sqrt{M}} \times \sum_{k=1}^{\log _{2} \sqrt{M}\left(1-2^{-k}\right) \sqrt{M}-1} \sum_{i=0}^{\sqrt{M}}\{(-1) & \frac{i \times 2^{k-1}}{\sqrt{M}\rfloor}\left(2^{k-1}-\left\lfloor\frac{i \times 2^{k-1}}{\sqrt{M}}+\frac{1}{2}\right\rfloor\right) \\
& \times Q\left((2 i+1) \times \sqrt{\left.\left.\frac{6 \log _{2} M}{2(M-1)} \times \frac{E_{b}}{N_{0}}\right)\right\}},\right.
\end{aligned}
$$


where the Q-function [119] defined as

$$
Q(x)=\frac{1}{2} \operatorname{erfc}\left(\frac{x}{\sqrt{2}}\right)
$$

is used to compute the error probability of the modulation scheme, with $x$ to be different for each modulation scheme. The complementary error function, denoted by erf $c$ is given by

$$
\operatorname{erfc}(x)=1-\operatorname{erf}(x)
$$

where the error function $\operatorname{erf}[68]$ is given by

$$
\operatorname{erf}(x)=\frac{2}{\sqrt{\pi}} \int_{0}^{x} e^{-t^{2}} d t
$$

As mentioned earlier, $E_{b} / N_{0}$ is the normalized $S I N R$ measure and is given (in dB) by

$$
\frac{E_{b}}{N_{0}}=S I N R-10 \times \log _{10} \frac{R_{b}}{B W}
$$

where $R_{b}$ is the bit rate in bits per second and for a given modulation type with $k=\log _{2} M$ bits per symbol and symbol rate, denoted by $\mathrm{SR}$, it is computed as

$$
R_{b}=k \times S R,
$$

where given the fact that each sub-carrier is able to carry data at a maximum rate of $15 \mathrm{ksps}$ (kilo symbols per second) and our assumption of $B \mathrm{~W}=10 \mathrm{MHz}$ (i.e., 600 sub-carrier), $S R$ is given as $15 \times 10^{3} \times 600=9 M$ sps. Now, by having 
$B E R$ computed as explained above, $B L E R$ is computed as follows:

$$
B L E R=1-(1-B E R)^{T B S},
$$

where the transport block size $T B S$ specifies the number of information bits in each block.

As demonstrated in Figure 5.16, we name a segment the set of four transport blocks carrying identical data. From Eq. 5.14, the SER is given by

$$
S E R=\prod_{i=1}^{4} B L E R_{i}
$$

Accordingly, for a message composed of $n$ number of segments, the MER is given by

$$
M E R=1-\prod_{i=1}^{n}\left(1-S E R_{i}\right) .
$$

Message Delivery Ratio (MDR) Having obtained MER as in Eq. 5.16, we also define MDR for a given BG $j$ as the probability for a message to be received correctly by all the receivers of the $\mathrm{BG} j$ which is given by

$$
M D R_{j}=\prod_{i=1}^{r}\left(1-M E R_{i}\right)
$$

where $r$ represents the number of receivers of the $\mathrm{BG} j$. Accordingly, the average MDR for all active BGs is given by

$$
M D R=\frac{\sum_{j=1}^{\# B G s} M D R_{j}}{\# B G s} .
$$

\subsubsection{Numerical results}

In this section, results of evaluating system performance, in terms of the metrics introduced in 5.6.1.1 are presented given the modeling scenario 2. Note that being within $95 \%$ confidence intervals, the results for each numerical setting are obtained by averaging over fifty random topologies of a given activation rate of 
broadcast groups. For some graphs, logarithmic scales are used as a means of better distinguishing small differences in values.

Figure 5.17a demonstrates MER for random and spatial resource reuse approaches in a single cell scenario. One may note significant increase of MER values by increasing activation rate of broadcast groups, in the random resource reuse scenario. This is while our proposed spatial resource reuse approach is substantially more robust and outperforms the random resource reuse approach by significantly suppressing interference.

Figure 5.17b demonstrates MER for the same scenario in a three-dimensional graph, where error rates are separately visualized per defined distance bin. We have divided the target coverage range of $1 \mathrm{~km}$ into $10 \mathrm{bins}$, each of $0.1 \mathrm{~km}$ (denoted by $R X-T X$ distance axis label in Figure $5.17 \mathrm{~b}$ ). While MER is almost negligible for the proposed spatial resource reuse approach, for the random resource reuse approach one may notice significant increase of MER, not only against increasing activation rate of broadcast groups, but also as the receivers fall further away from the transmitter.

Figure 5.18a demonstrates MDR for random and spatial resource reuse approaches, for the same setting as in Figure 5.17. In Figure 5.18b, MDR is shown for the same scenario in surface plots, against increasing activation rate of broadcast groups and $R X-T X$ distance. The distinct difference between delivery ratios of two approaches indicates the performance improvement achievable by our proposed approach. Further, in the random resource reuse approach one may notice how the disruptive impact of increasing activation rate of broadcast groups is of higher order (compared to the spatial resource reuse approach) at farther distances from the transmitter. This is due to the increasing number of interfering transmissions and lower levels of the received SINR.

Recalling Figure 5.13 in Section 5.5.1 (based on the first modeling approach introduced in Section 5.5), one can notice similar results with what observed in Figure 5.18a (based on the second modeling approach introduced in Section 5.6) for the spatial resource reuse approach in the single cell scenario. Note that little drop in MDR values in Figure 5.18a under rather higher loads is due to increasing number of interfering transmissions and also adopting a modulation scheme of higher order which results in lower MDR values.

Counterparts of Figure 5.17 and Figure 5.18 for the double cell scenario are demonstrated in Figure 5.19 and Figure 5.20, respectively. As expected, intensified impact of interfering transmissions from the second cell is confirmed by the figures. Nevertheless, the proposed spatial resource reuse approach has succeeded to significantly outperform the random resource reuse approach and provide reasonable performance. In particular, for low to moderate activation rates of BGs, one may note the robustness of the proposed spatial resource reuse approach against increasing distance between the transmitter and receivers, by 


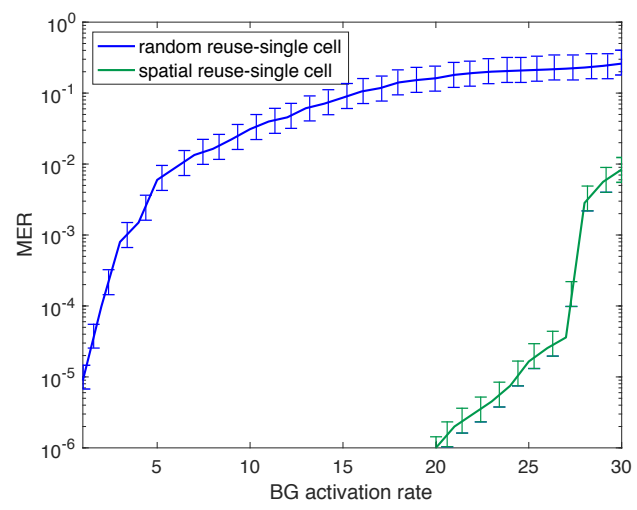

(a) MER.

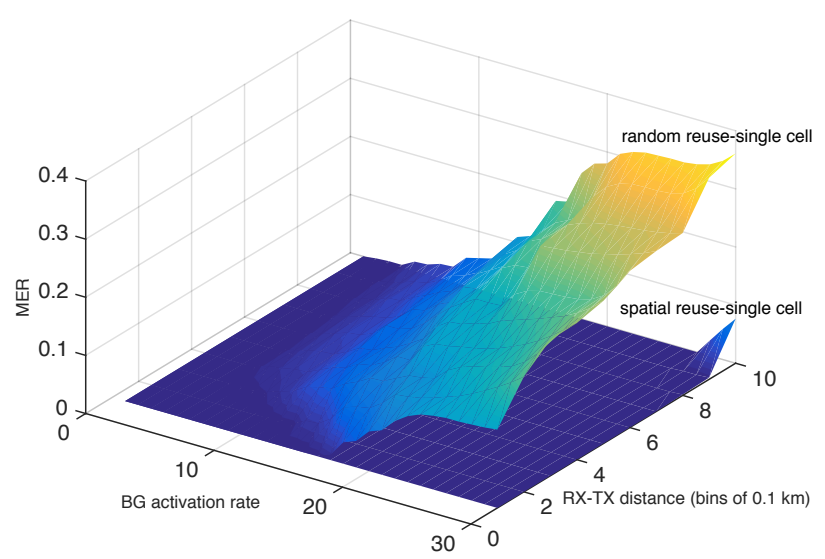

(b) Distance-based MER.

Figure 5.17: MER single cell scenario, PoolPeriod=120ms, MS=1000Byte.

keeping MER and MDR at reasonable levels. This provides important insight regarding adjusting the target coverage in order to meet the performance requirements of the application of interest.

By comparing Figure 5.13 and Figure 5.18a with respect to the spatial resource reuse approach in the double cell scenario, one can notice similar results for the two modeling approaches (introduced in Section 5.5 and Section 5.6) under rather moderate loads (i.e., $N_{V_{T}}$ ). As the load increases though, we observe considerably lower MDR values for the second modeling approach. Assuming similar impact of interfering transmissions regardless of the considered modeling 


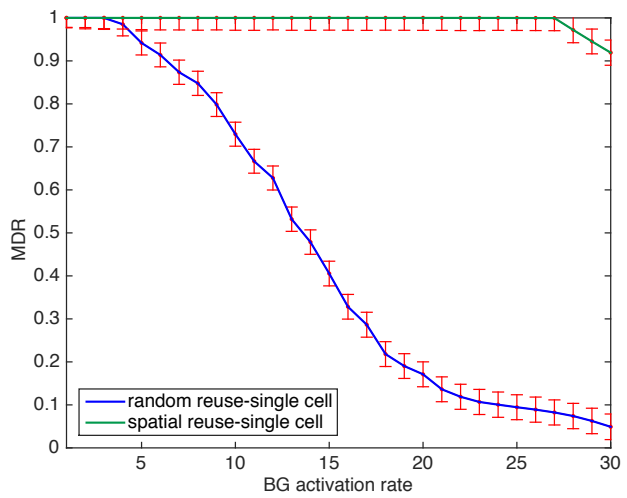

(a) MDR.

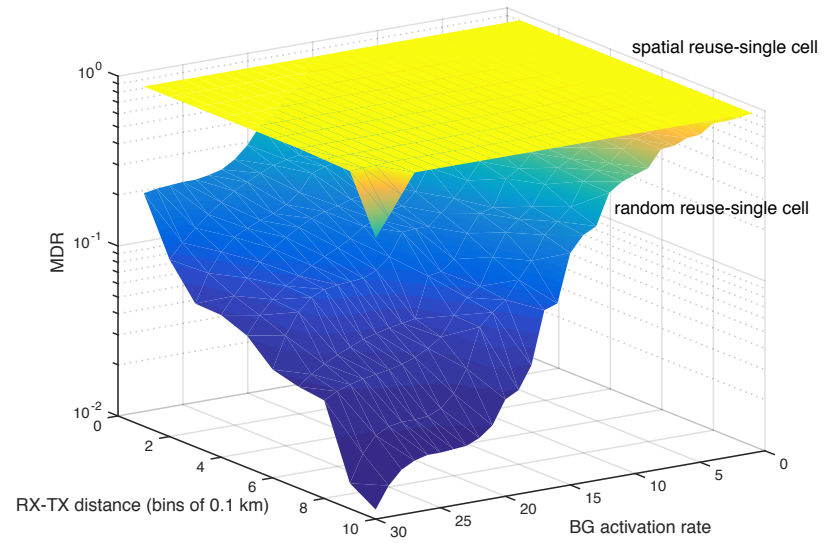

(b) Distance-based MDR.

Figure 5.18: MDR single cell scenario, PoolPeriod=120ms, MS=1000Byte.

approach, this is explicable by adopting (in the second modeling approach) modulation schemes of higher order, as a means of compensating reduced number of RBs per transmitter under high loads and consequently fitting the message transmission within the allocated PoolPeriod which comes at the cost of lower MDR values. Whereas in the first modeling approach we did not limit transmission of multiple messages into one PoolPeriod and neither did we consider adaptive modulation. As a consequence, given reduced number of RBs per 


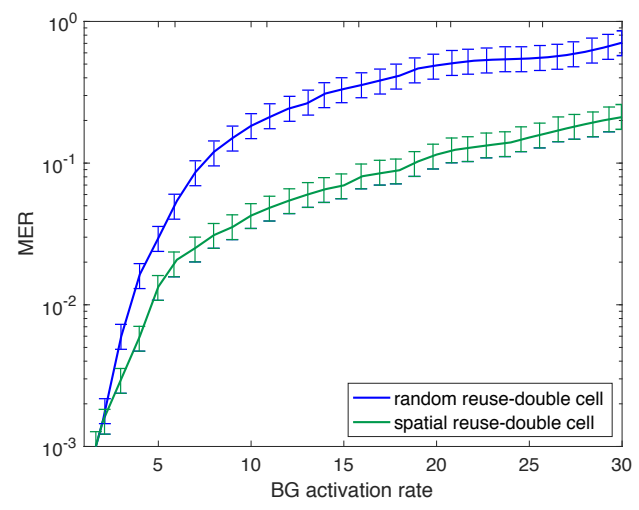

(a) MER.

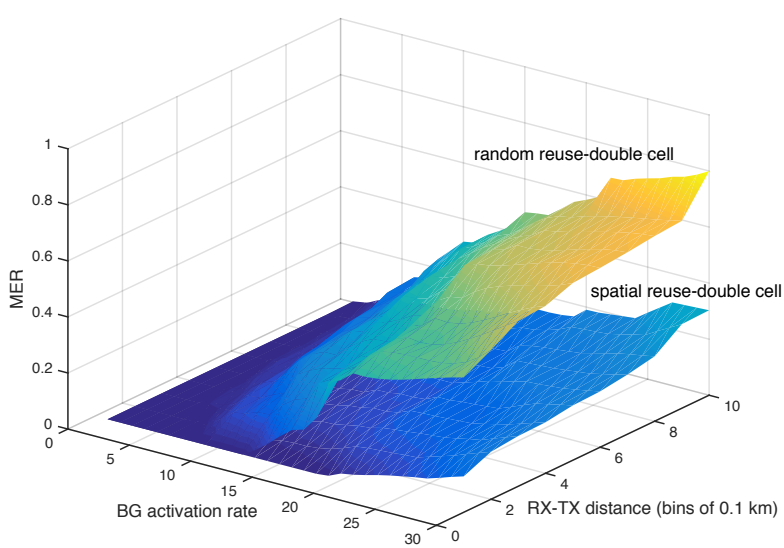

(b) Distance-based MER.

Figure 5.19: MER double cell scenario, PoolPeriod=120ms, MS=1000Byte.

transmitter under high loads, transmissions are subject to more delay, due to being spread over multiple PoolPeriods.

Note that depending on the use case, either of the modeling approaches may suit expected requirements. For instance, the first modeling approach may be employed in case large data files (e.g., large maps and videos) are supposed to be broadcast. While in case of safety critical applications where a single message is supposed to be broadcast in short time, the second modeling approach is a better option. 


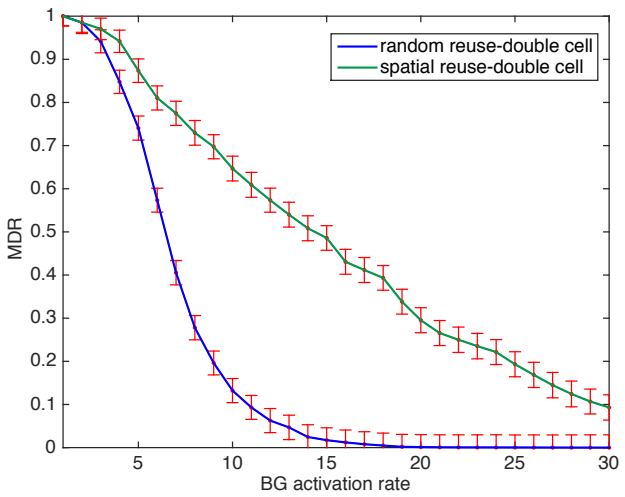

(a) MDR.

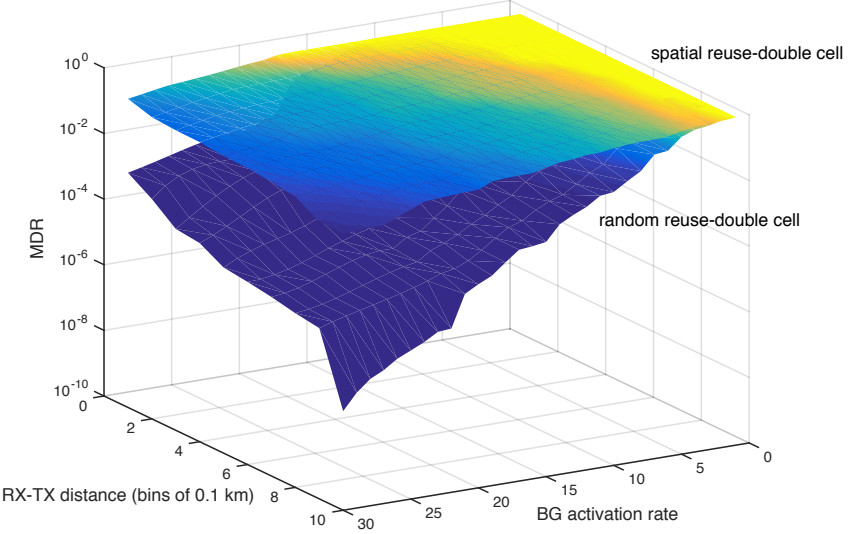

(b) Distance-based MDR.

Figure 5.20: MDR double cell scenario, PoolPeriod=120ms, MS=1000Byte.

\subsection{Concluding remarks}

In this chapter, we studied D2D vehicular broadcast, overlaying the cellular network, with the main focus on radio resource allocation. Given the dynamicity of a vehicular network, a fixed resource management scheme cannot meet the performance of ITS applications with stringent reliability requirement. Therefore, we proposed a resource allocation scheme which is adaptive in two aspects. That is, by considering the fourth generation of the mobile networking system 
as the basis, we developed a resource allocation scheme based on load control and spatial resource reuse.

We evaluated the performance of the proposed scheme in the context of two modeling approaches featuring different characteristics and application requirements. By taking effective factors as inputs into the models, we quantified the performance of D2D vehicular broadcast in terms of relevant measures.

Extensive numerical results of various scenarios confirm the flexibility and reliability of the proposed resource management scheme, in comparison with the baseline approach of random resource reuse. These observations provide insight on the network design regarding the optimal allowed data rate and resource assignment according to application requirements.

As mentioned earlier in this paper and also confirmed by our results, radio resource allocation is an essential factor in realizing resource efficient and reliable D2D communication. Based on the obtained results, we think that D2D communication technology is a promising alternative / complement to the IEEE 802.11p standard for safety ITS applications, demanding highly reliable communication. However, further research is required to confirm this conclusion for large-scale real world deployments.

Our approach is not limited to a specific network setting and as a result can be further extended by introducing more details into the modeling. For instance, one could think of a scenario demanding a longer broadcast time scale and taking mobility into account. Further, D2D resource allocation in a multicell scale, for underlay and overlay schemes, can be considered as a relevant direction for future studies. 


\section{Conclusions}

In this chapter, we conclude the thesis by first recalling the main research objective and questions. We continue by summarizing the contributions of our research and the main conclusions drawn. We end the chapter by providing directions for future research.

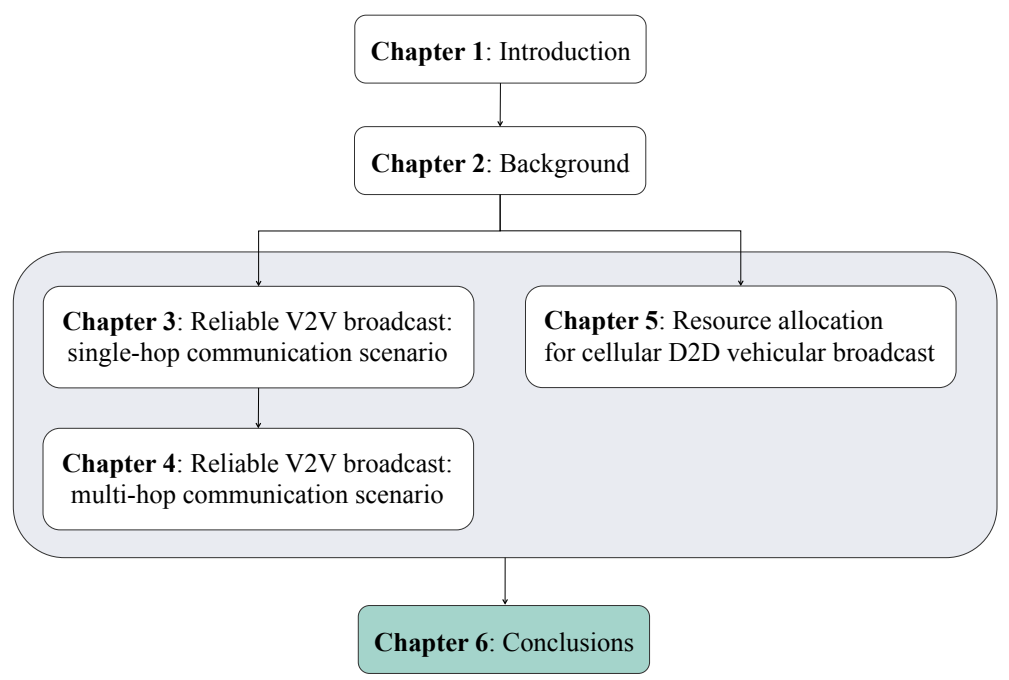

This chapter is organized as follows:

- Section 6.1 presents the main contributions and conclusions of this thesis.

- Section 6.2 presents directions for future research. 


\subsection{Contributions and Conclusions}

In this section, we list the main contributions and findings of this thesis, per chapter. For this, we first recall our main goal as

[...] to model and analyze reliability of broadcasting in vehicular networks, and identify and validate solutions for its improvement,

that has led us to establish our research questions introduced in Section 1.2, reminded as follows.

- How can a system for vehicular broadcasting be modeled, with a reasonable level of abstraction?

- How and in terms of which metrics can performance of the system be evaluated using the model?

- How can performance of vehicular broadcast be improved?

In what follows, we continue by summarizing the scope and conclusions of each chapter.

\section{Chapter 2. Vehicular networking}

As a preliminary step towards addressing the research questions, we first presented in Chapter 2 an introduction to vehicular networking and its role in realizing ITS applications, as a general background on the topic of this thesis. In this chapter, we discussed the communication technologies and networking protocols defined in the context of vehicular networking. We elaborated on IEEE 802.11p-based DSRC and 5G-based D2D communication technologies, as two main communication alternatives for vehicular broadcasting studied in this thesis. We further continued by taking a closer look on data forwarding categories in vehicular networks and finally highlighted the main requirements of vehicular communications towards fulfilling high performance ITS applications.

All our research questions are equally covered in three chapters. That is, modeling, evaluation and further improvement of performance of vehicular broadcasting have been focused on in these chapters, as summarized by their contributions and conclusions below.

Chapter 3. Reliable vehicular broadcast: a single-hop scenario 
In Chapter 3, we proposed a reliability assurance mechanism where based on a sequence checking module receiver vehicles are able to verify whether they have correctly received broadcast data and accordingly take the error recovery initiative by sending retransmission requests. Accordingly, vehicles in possession of requested data, reply to the requests. We developed an analytical model of a single-hop broadcast communication setting where we applied the proposed mechanism and further evaluated its performance in terms of key performance indicators such as delivery ratio and overhead of error recovery. The capability of our analytical model in capturing the functionality of the proposed reliability mechanism was verified via simulations. We have demonstrated that for rather small to medium size network settings the proposed mechanism can be safely used to assure broadcast reliability, without imposing significant recovery overhead. It is for further investigation how it evolves in large-scale practical deployments with highly dynamic and adverse conditions of high loss probabilities and colliding concurrent transmissions.

\section{Chapter 4. Reliable vehicular broadcast: a multi-hop scenario}

As an extension to the work presented in Chapter 3, in Chapter 4 we focused on a multi-hop broadcast setting. For this, we first modeled broadcasting over multiple hops. Accordingly and by means of an extensive analytical modeling, the reliability assurance mechanism was assessed for recovering failures that may have happened during broadcasting. Though analytical models come with some simplifying assumptions and abstractions from the real system, we have shown the strength of our model in reflecting main system functionality via verification by simulations. Further, the proposed mechanism proved its efficiency in realizing reliable broadcasting for rather small to medium size network settings. Via defining relevant measures and quantifying them through various experiments, we have shown that the proposed mechanism is able to provide satisfactory performance. Though, in order to obtain more solid insight on robustness of the proposed mechanism in providing satisfactory performance in large practical settings of varying network conditions, a more realistic emulation platform or a real-world test case is required.

\section{Chapter 5. Resource allocation for cellular D2D vehicular broadcast}

We also looked for other cutting-edge communication technologies as alternatives to the IEEE 802.11p standard, which has some inherent performance limitations in terms of scalability and communication reliability. Utilizing emerging cellular D2D communication technology for vehicular broadcasting purpose, has 
been our focus in Chapter 5. Benefiting from central management capabilities of the cellular system infrastructure, D2D communication technology has been discussed as a promising technology for vehicular broadcasting. One of the core challenges of D2D communication technology, which is critical in realizing reliable communications, is resource allocation. The cellular radio resource spectrum is to be utilized both for conventional cellular and D2D communications. In this context, reuse of radio resources by D2D emerges due to the limited system bandwidth. Reuse of resources must follow insightful principles, otherwise the expected performance cannot be reached, due to interfering transmissions over the same resource. Hence, we focused on this aspect by proposing a D2D resource allocation mechanism with adaptive functionality based on the varying network circumstance. By considering reasonable simplifying assumptions and abstractions from the real-world implementation, we modeled a D2D vehicular broadcasting setting in the current $4 \mathrm{G}$ cellular system, relying on our proposed resource allocation mechanism. Extensive numerical experiments demonstrated the efficiency of our proposed mechanism in adapting to the varying network states (topology and density) and hence providing good performance. In particular, obtained results demonstrated the performance gain of our proposed mechanism in comparison with the baseline random resource allocation mechanism, with significant difference. The obtained results emphasize the necessity of insightful D2D resource allocation. Otherwise, promising gains offered by D2D communication can easily disappear. Similar to our earlier research in this thesis, verifying efficiency of the proposed mechanism in large-scale deployments calls for more elaborate tests, as close as possible to practice, in terms of network size and considered system details.

\subsection{Future research directions}

In this section, we set out research directions to extend the work presented in this thesis, as follows.

In Chapter 3 and Chapter 4 of this thesis, we proposed a reliability assurance mechanism based on a sequence checking module and evaluated it in the context of IEEE 802.11p based single-hop and multi-hop vehicular broadcasting. Introducing more details into the proposed modeling approach would be the next obvious step. For instance, long run operation of the proposed reliability assurance mechanism (for instance, in case of the events with long-lasting impact to be communicated) can be considered along with suitable mobility and propagation models. Over such long time spans the target receiver group for broadcast data varies. Such that some vehicles leave the area, while new ones 
enter. This will have impact on the chances of acquiring missing broadcast data as a result of request / reply iterations among vehicles.

In Chapter 5, we proposed a resource allocation scheme, aiming network load and interference management in the context of single-hop D2D vehicular broadcasting. Further research can be done with respect to the performance gain obtained by applying the proposed E2E reliability assurance mechanism from Chapter 3 and Chapter 4 in this context.

Another direction to consider could be extending the target coverage area of vehicular broadcasting either via transmissions over multiple hops or relying on cellular network infrastructure and investigate how differently the proposed resource allocation mechanism performs in these two contexts.

Reducing the D2D transmission power in case of high network loads can be considered as an alternative to adaptive modulation as used in Chapter 5 . Their performance difference in providing broadcast reliability can be further studied.

In this thesis, we considered the IEEE 802.11p standard and D2D as two communication technologies for vehicular broadcasting. A logical next step would be using these alternatives in combination as complementary technologies in a hybrid communication setting for vehicular broadcast, as a means of benefiting from characteristics of both technologies and meet stringent requirements of ITS applications. A possible scenario could be utilizing IEEE 802.11p-based broadcast in lower network densities with no management load on the network infrastructure and relying on D2D-based broadcast with management support from the network infrastructure in higher network densities where the IEEE 802.11p standard fails to scale up and meet satisfactory performance. 



\section{Open Data Management}

In Table A.1, this appendix provides links to the source code developed for this thesis, on a per-chapter basis.

Table A.1: Description and URLs of the source codes used per chapter.

\begin{tabular}{|l|l|l|}
\hline Chapter & Brief Description & Source Code \\
\hline \hline Chapter 3 & $\begin{array}{l}\text { The Mathematica scripts for the developed analytical model of } \\
\text { the proposed reliability mechanism for the single-hop broad- } \\
\text { cast scenario. }\end{array}$ & $\begin{array}{l}\text { https://github.com/ } \\
\text { mg/reliable-single- } \\
\text { hop-scenario }\end{array}$ \\
\hline Chapter 4 & $\begin{array}{l}\text { The Mathematica script for the developed analytical model of } \\
\text { the multi-hop broadcast scenario. }\end{array}$ & $\begin{array}{l}\text { https://github. } \\
\text { com/mg/multi-hop- } \\
\text { broadcast-scenario }\end{array}$ \\
\hline Chapter 4 & $\begin{array}{l}\text { The Mathematica script for the developed analytical model of } \\
\text { the proposed reliability mechanism for the multi-hop broad- } \\
\text { cast scenario. }\end{array}$ & $\begin{array}{l}\text { https://github.com/ } \\
\text { hop-brobadia-multi- } \\
\text { hop-broadcast- } \\
\text { scenario }\end{array}$ \\
\hline Chapter 5 & $\begin{array}{l}\text { The Matlab scripts for the proposed D2D resource allocation } \\
\text { mechanism and the modeling scenarios for evaluation of the } \\
\text { mechanism. }\end{array}$ & $\begin{array}{l}\text { https://github.com/ } \\
\text { mg/D2D }\end{array}$ \\
\hline
\end{tabular}





\section{Bibliography}

[1] CUBE. [Online; accessed 14-September-2017].

[2] JiST / SWANS, Java in Simulation Time / Scalable Wireless Ad hoc Network Simulator. [Online; accessed 15-September-2017].

[3] OMNeT ++ Network Simulation Framework. [Online; accessed 15-September2017].

[4] OPNET, Application and Network Performance. [Online; accessed 15September-2017].

[5] QualNet Network Simulator Software. [Online; accessed 15-September-2017].

[6] The Network Simulator - ns-2. [Online; accessed 15-September-2017].

[7] The Network Simulator - ns-3. [Online; accessed 15-September-2017].

[8] Veins, Vehicles in Network Simulation. [Online; accessed 15-September-2017].

[9] VISUM. [Online; accessed 14-September-2017].

[10] Simulation of Urban MObility (SUMO), 2000. [Online; accessed 14-September2017].

[11] VanetMobiSim, 2006. [Online; accessed 14-September-2017].

[12] Car-to-Car Communication Consortium (C2C-CC) Manifesto, 2007. [Online; accessed 17-July-2014].

[13] Generic Mobility Simulation Framework (GMSF), 2007. [Online; accessed 14September-2017].

[14] Traffic Simulation Systems (TSS):Aimsun traffic modelling software, 2007. [Online; accessed 14-September-2017].

[15] IEEE standard for information technology- local and metropolitan area networks- part 11: WLAN MAC and PHY specifications amendment 6: Wave. IEEE Std 802.11p-2010, pages 1-51, July 2010.

[16] Mobility 2.0 - co-operative ITS systems for enhanced electric vehicle mobility. 7th Framework Programme for research, grant agreement no. 314129., 2014. 
[17] Global status report on road safety 2015. Technical report, World health Organization (WHO), 2015.

[18] 5G Infrastructure Association (5G PPP). 5G PPP use cases and performance evaluation models, April 2016. https://5g-ppp.eu/wp-content/uploads/2014/ 02/5G-PPP-use-cases-and-performance-evaluation-modeling_v1.0.pdf.

[19] 5G infrastructure association (5G PPP). View on $5 G$ Architecture, Jan 2018. https://5g-ppp.eu/wp-content/uploads/2018/01/5G-PPP-5GArchitecture-White-Paper-Jan-2018-v2.0.pdf.

[20] 3rd Generation Partnership Project. 3GPP specification: 36.843; Technical Specification Group Radio Access Network; Study on LTE Device to Device Proximity Services; Radio Aspects Stage 2. Technical report, 3rd Generation Partnership Project, 2014.

[21] 3rd Generation Partnership Project. 3GPP specification: 23.303; Technical Specification Group Services and System Aspects; Proximity-based services (ProSe); Stage 2. Technical report, 3rd Generation Partnership Project, 2016.

[22] 3rd Generation Partnership Project. 3GPP specification: 36.213; Technical Specification Group Radio Access Network; Evolved Universal Terrestrial Radio Access (E-UTRA); Physical layer procedures. Technical report, 3rd Generation Partnership Project, September 2016.

[23] 3rd Generation Partnership Project. 3GPP specification: 36.942; Technical Specification Group Radio Access Network; Evolved Universal Terrestrial Radio Access (E-UTRA); Radio Frequency (RF) system scenarios . Technical report, 3rd Generation Partnership Project, 2016.

[24] 3rd Generation Partnership Project. 3GPP specification: 36.300; Technical Specification Group LTE; Evolved Universal Terrestrial Radio Access (E-UTRA) and Evolved Universal Terrestrial Radio Access Network (E-UTRAN); Overall description; Stage 2. Technical report, 3rd Generation Partnership Project, 2017.

[25] 3rd Generation Partnership Project. 3GPP specification:30.03U; Selection procedures for the choice of radio transmission technologies of the UMTS. Technical report, 3rd Generation Partnership Project, V3.2.0, 1998.

[26] R. Akamatsu, M. Suzuki, T. Okamoto, K. Hara, and H. Shigeno. Adaptive delay-based geocast protocol for data dissemination in urban VANET. In Mobile Computing and Ubiquitous Networking (ICMU), 2014 Seventh International Conference on, pages 141-146, Jan 2014.

[27] Saif Al-Sultan, Moath M. Al-Doori, Ali H. Al-Bayatti, and Hussien Zedan. A comprehensive survey on vehicular ad hoc network. Journal of Network and Computer Applications, 37:380 - 392, 2014. 
[28] S. Allal and S. Boudjit. Geosuz: A geocast routing protocol in sub-zors for VANETs. In Vincent Guyot, editor, Advanced Infocomm Technology, volume 7593 of Lecture Notes in Computer Science. 2013.

[29] A. Asadi, Q. Wang, and V. Mancuso. A survey on device-to-device communication in cellular networks. IEEE Communications Surveys Tutorials, 16(4):18011819, Fourth quarter 2014.

[30] D. Astely, E. Dahlman, G. Fodor, S. Parkvall, and J. Sachs. Lte release 12 and beyond [accepted from open call]. IEEE Communications Magazine, 51(7):154160, July 2013.

[31] T. Atechian and L. Brunie. DG-CastoR for query packets dissemination in VANET. In Mobile Ad Hoc and Sensor Systems, 2008. MASS 2008. 5th IEEE International Conference on, pages 547-552, Sept 2008.

[32] A. Sudhir Babu and KV. Sambasiva Rao. Evaluation of ber for AWGN, Rayleigh and Rician fading channels under various modulation schemes. International Journal of Computer Applications, 26(9), 2011.

[33] A Bachir and A. Benslimane. A multicast protocol in ad hoc networks intervehicle geocast. In Vehicular Technology Conference, 2003.

[34] K. Bilstrup, E. Uhlemann, E. G. Strom, and U. Bilstrup. Evaluation of the IEEE 802.11p MAC method for V2V communication. In Vehicular Technology Conference (VTC), 2008. IEEE 68th, pages 1-5, 2008.

[35] M. Botsov, M. Klugel, W. Kellerer, and P. Fertl. Location dependent resource allocation for mobile device-to-device communications. In 2014 IEEE Wireless Communications and Networking Conference (WCNC), pages 1679-1684, April 2014 .

[36] Giammarco Cecchini, Alessandro Bazzi, Barbara Masini, and Alberto Zanella. Performance comparison between IEEE 802.11p and LTE-V2V in-coverage and out-of-coverage for cooperative awareness. In Vehicular Networking Conference (VNC), 2017 IEEE, pages 109-114. IEEE, 2017.

[37] Si-Ho Cha, Keun-Wang Lee, , and Hyun-Seob Cho. Grid-based predictive geographical routing for inter-vehicle communication in urban areas. Distributed Sensor Networks, 2012, Jan 2012.

[38] Moumena Chaqfeh, Abderrahmane Lakas, and Imad Jawhar. A survey on data dissemination in vehicular ad hoc networks. Vehicular Communications, 1(4):214 $-225,2014$.

[39] Rex Chen, Wen-Long Jin, and Amelia Regan. Broadcasting safety information in vehicular networks: issues and approaches. IEEE network, 24(1), 2010. 
[40] Yuh-Shyan Chen, Yun-Wei Lin, and Sing-Ling Lee. A mobicast routing protocol in vehicular ad-hoc networks. Mob. Netw. Appl., 15(1):20-35, February 2010.

[41] F. Chiti and R. Fantacci. An efficient harq scheme for applications in multicast communication systems. WCM, July 2013.

[42] Kyongkuk Cho and Dongweon Yoon. On the general BER expression of oneand two-dimensional amplitude modulations. IEEE Transactions on Communications, 50(7):1074-1080, July 2002.

[43] Ming-Chin Chuang and Meng Chang Chen. Deep: Density-aware emergency message extension protocol for VANETs. IEEE Transactions on Wireless Communications, 12(10):4983-4993, October 2013.

[44] M. Dixit, R. Kumar, and A. K. Sagar. Vanet: Architectures, research issues, routing protocols, and its applications. In 2016 International Conference on Computing, Communication and Automation (ICCCA), pages 555-561, April 2016.

[45] K. Doppler, M. Rinne, C. Wijting, C. B. Ribeiro, and K. Hugl. Device-to-device communication as an underlay to LTE-Advanced networks. IEEE Communications Magazine, 47(12):42-49, Dec 2009.

[46] Amit Dua, Neeraj Kumar, and Seema Bawa. A systematic review on routing protocols for vehicular ad hoc networks. Vehicular Communications, 1(1):33 52,2014 .

[47] Richard Gilles Engoulou, Martine Bellaiche, Samuel Pierre, and Alejandro Quintero. VANET security surveys. Computer Communications, 44:1-13, 2014.

[48] European Telecommunications Standards Institute Technical Specification (ETSI TS). "202-663 v1.1.0: Intelligent Transport Systems (ITS); European profile standard for the physical and medium access control layer of Intelligent Transport Systems operating in the $5 \mathrm{GHz}$ frequency band". Technical report, 2010 .

[49] European Telecommunications Standards Institute Technical Specification (ETSI TS). "302-665 v1.1.1: Intelligent Transport Systems (ITS); Communication Architecture". Technical report, 2010.

[50] European Telecommunications Standards Institute Technical Specification (ETSI TS). "102 636-4-1 v1.1.1: Intelligent Transport Systems (ITS); Vehicular Communications; GeoNetworking". Technical report, 2011.

[51] European Telecommunications Standards Institute Technical Specification (ETSI TS). "102 687 v1.1.1: Intelligent Transport Systems (ITS); Decentralized Congestion Control Mechanisms for Intelligent Transport Systems operating in the $5 \mathrm{GHz}$ range; Access layer part". Technical report, 2011. 
[52] European Telecommunications Standards Institute Technical Specification (ETSI TS). "302 636-5-1 v1.2.0: Intelligent transport systems (ITS); vehicular communications; geonetworking; part 5: Transport protocols;sub-part 1: Basic transport protocol". Technical report, 2013.

[53] European Telecommunications Standards Institute Technical Specification (ETSI TS). "302 637-2 v1.3.2: Intelligent Transport Systems (ITS); vehicular communications; basic set of applications;part 2: Specification of cooperative awareness basic service". Technical report, 2014.

[54] European Telecommunications Standards Institute Technical Specification (ETSI TS). "302 637-3 v1.2.2: Intelligent Transport Systems (ITS); vehicular communications; basic set of applications; part 3: Specifications of decentralized environmental notification basic service". Technical report, 2014.

[55] C. E. Eze, S. Zhang, and E. Liu. Message dissemination reliability in vehicular networks. In 2015 21st International Conference on Automation and Computing (ICAC), pages 1-6, Sept 2015.

[56] Y.P. Fallah, Ching-Ling Huang, Raja Sengupta, and H. Krishnan. Analysis of information dissemination in vehicular ad-hoc networks with application to cooperative vehicle safety systems. Vehicular Technology, IEEE Transactions on, 60(1):233-247, Jan 2011.

[57] Y.P. Fallah, Ching-Ling Huang, Raja Sengupta, and H. Krishnan. Analysis of information dissemination in vehicular ad-hoc networks with application to cooperative vehicle safety systems. Vehicular Technology, IEEE Transactions on, 60(1):233-247, Jan 2011.

[58] Martin Fellendorf and Peter Vortisch. Microscopic traffic flow simulator VISSIM. In Fundamentals of traffic simulation, pages 63-93. Springer, 2010.

[59] A. Festag. Standards for vehicular communication-from IEEE 802.11p to 5G. Elektrotechnik und Informationstechnik, 132(7):409-416, 2015.

[60] G. Fodor, E. Dahlman, G. Mildh, S. Parkvall, N. Reider, G. Miklós, and Z. Turányi. Design aspects of network assisted device-to-device communications. IEEE Communications Magazine, 50(3):170-177, March 2012.

[61] R. Fracchia and M. Meo. Analysis and design of warning delivery service in intervehicular networks. IEEE Transactions on Mobile Computing, 7(7):832845 , July 2008.

[62] Shamil I. Galiev and Maria S. Lisafina. Linear models for the approximate solution of the problem of packing equal circles into a given domain. European Journal of Operational Research, 230(3):505 - 514, 2013. 
[63] M. Gholibeigi, M. Baratchi, H. van den Berg, and G. Heijenk. Towards reliable multi-hop broadcast in VANETs: An analytical approach. In 2016 IEEE Vehicular Networking Conference (VNC), pages 1-8, Dec 2016.

[64] M. Gholibeigi and G. Heijenk. Analysis of multi-hop broadcast in vehicular ad hoc networks: A reliability perspective. In 2016 Wireless Days (WD), pages 1-8, March 2016.

[65] M. Gholibeigi, G. Heijenk, D. Moltchanov, and Y. Koucheryavy. Analysis of a receiver-based reliable broadcast approach for vehicular networks. In IEEE Vehicular Networking Conference (VNC), 2014.

[66] M. Gholibeigi, G. Heijenk, D. Moltchanov, and Y. Koucheryavy. Analysis of a receiver-based reliable broadcast approach for vehicular networks. Ad Hoc Networks, 37, Part 1:63 - 75, 2016. Special Issue on Advances in Vehicular Networks.

[67] M. Gholibeigi, N. Sarrionandia, M. Karimzadeh, M. Baratchi, H. van den Berg, and G. Heijenk. Reliable vehicular broadcast using $5 \mathrm{G}$ device-to-device communication. In IEEE/IFIP Wireless and Mobile Networking Conference (WMNC), $201 \%$.

[68] I.S. Gradshteyn and I.M. Ryzhik. Table of Integrals, Series, and Products. Elsevier Science, 2014.

[69] Abolhassan Halati, Henry Lieu, and Susan Walker. CORSIM-corridor traffic simulation model. In Traffic congestion and traffic safety in the 21st century: Challenges, innovations, and opportunities, 1997.

[70] Jérôme Härri, Fethi Filali, Christian Bonnet, and Marco Fiore. VanetMobiSim: generating realistic mobility patterns for VANETs. In Proceedings of the 3rd international workshop on Vehicular ad hoc networks, pages 96-97. ACM, 2006.

[71] S.A. Hassan and M.A. Ingram. Modeling of a cooperative one-dimensional multihop network using quasi-stationary markov chains. In Global Telecommunications Conference (GLOBECOM 2010), 2010 IEEE, pages 1-5, Dec 2010.

[72] B. Hassanabadi and S. Valaee. Reliable periodic safety message broadcasting in VANETs using network coding. Wireless Communications, IEEE Transactions on, 13(3):1284-1297, March 2014.

[73] Institute of Electrical and Electronics Engineers (IEEE). "IEEE standard for Information Technology - Telecommunications and Information Exchange Between Systems Local and Metropolitan Area networks - Specific Requirements Part 11: Wireless LAN Medium Access Control (MAC) and Physical Layer (PHY) Specifications". Technical report, 2012.

[74] S.Reed Irving and X. Chen. Error Control Coding for Data Networks. Springer, 1999. 
[75] E.T. Jaynes and G.L. Bretthorst. Probability Theory: The Logic of Science. Cambridge University Press, 2003.

[76] G. Karagiannis, O. Altintas, E. Ekici, G. Heijenk, B. Jarupan, K. Lin, and T. Weil. Vehicular networking: A survey and tutorial on requirements, architectures, challenges, standards and solutions. IEEE Communications Surveys Tutorials, 13(4):584-616, Fourth 2011.

[77] J. Kemeny and J. Snell. Finite Markov Chains, volume 40 of Undergraduate Texts in Mathematics. Springer-Verlag, 1960.

[78] M. Khabazian, S. Aissa, and M. Mehmet-Ali. Performance modeling of safety messages broadcast in vehicular ad hoc networks. Intelligent Transportation Systems, IEEE Transactions on, 14(1):380-387, March 2013.

[79] Vaishali Khairnar and Ketan Kotecha. Performance of vehicle-to-vehicle communication using IEEE $802.11 \mathrm{p}$ in vehicular ad-hoc network environment. arXiv preprint arXiv, 1304.335\%, 2013.

[80] Maria Kihl, Mihail L. Sichitiu, and Harshvardhan P. Joshi. Design and evaluation of two geocast protocols for vehicular ad-hoc networks. In Journal of Internet Engineering, 2, 2008.

[81] Wouter Klein Wolterink, Geert Heijenk, and Hans van den Berg. Analytically modelling the performance of piggybacking on beacons in VANETs. In Proceedings of the Ninth ACM International Workshop on Vehicular Inter-networking, Systems, and Applications, VANET'12, pages 43-52, New York, NY, USA, 2012. ACM.

[82] F. Klingler, F. Dressler, and C. Sommer. IEEE 802.11p unicast considered harmful. In 2015 IEEE Vehicular Networking Conference (VNC), pages 76-83, Dec 2015.

[83] M. Koubek. Safety Data Dissemination Framework for Vehicular Networks. PhD thesis, Cork Institute of Technology, October 2010.

[84] A. Kwoczek, B. Rech, T. Hehn, T. Buburuzan, and R. Alieiev. 5G infrastructure association (5G PPP). $5 G$ Automotive Vision, October 2015. https://5g-ppp.eu/wp-content/uploads/2014/02/5G-PPP-WhitePaper-on-Automotive-Vertical-Sectors.pdf.

[85] A. Lakas and M. Shaqfa. Geocache: Sharing and exchanging road traffic information using peer-to-peer vehicular communication. In Vehicular Technology Conference (VTC Spring), IEEE 73rd, pages 1-7, May 2011.

[86] Jeng-Farn Lee, Chang-Sheng Wang, and Ming-Chin Chuang. Fast and reliable emergency message dissemination mechanism in vehicular ad hoc networks. In Wireless Communications and Networking Conference (WCNC), 2010 IEEE, pages 1-6, April 2010. 
[87] J. H. Lim, W. Kim, K. Naito, J. H. Yun, D. Cabric, and M. Gerla. Interplay between TVWS and DSRC: Optimal strategy for safety message dissemination in VANET. IEEE Journal on Selected Areas in Communications, 32(11):21172133, November 2014.

[88] Shu Lin and Daniel J Costello. Error control coding, volume 2. Prentice Hall Englewood Cliffs, 2004.

[89] X. Lin, J. G. Andrews, A. Ghosh, and R. Ratasuk. An overview of 3GPP device-to-device proximity services. IEEE Communications Magazine, 52(4):4048, April 2014.

[90] Congyi Liu and Chunxiao Chigan. RPB-MD: Providing robust message dissemination for vehicular ad hoc networks. Ad Hoc Networks, 2012.

[91] Y. Liu, D. Li, H. Guo, M. Baker, and F. C. Cheng. Resource allocation for device-to-device broadcast communication in cellular networks. In 2015 IEEE 26th Annual International Symposium on Personal, Indoor, and Mobile Radio Communications (PIMRC), pages 1178-1183, Aug 2015.

[92] Zhipeng Lu and Wenqi Huang. PERM for solving circle packing problem. Journal of Computers and Operations Research, 35(5):1742 - 1755, 2008. Part Special Issue: Algorithms and Computational Methods in Feasibility and Infeasibility.

[93] Xiaomin Ma, Jinsong Zhang, Xiaoyan Yin, and K.S. Trivedi. Design and analysis of a robust broadcast scheme for VANET safety-related services. IEEE Transactions on Vehicular Technology, 2012.

[94] Y. Malik, S.D. Bruda, B. Abdulrazak, and U. Tariq. Reliable communication protocol for inter-vehicular network. In Digital Information Management (ICDIM), 2013.

[95] I.R. Management Association. Transportation Systems and Engineering: Concepts, Methodologies, Tools, and Applications. 2015.

[96] I.R. Management Association. Transportation Systems and Engineering: Concepts, Methodologies, Tools, and Applications. 2015.

[97] M.N. Mariyasagayam, T. Osafune, and M. Lenardi. Enhanced multi-hop vehicular broadcast (MHVB) for active safety applications. In Telecommunications, 2007. ITST '07. 7th International Conference on ITS, pages 1-6, June 2007.

[98] Mohamed Nidhal Mejri, Jalel Ben-Othman, and Mohamed Hamdi. Survey on VANET security challenges and possible cryptographic solutions. Vehicular Communications, 1:53-66, 2014.

[99] Bassem Mokhtar and Mohamed Azab. Survey on security issues in vehicular ad hoc networks. Alexandria Engineering Journal, 54(4):1115-1126, 2015. 
[100] P. Muhlethaler, A. Laouiti, and Y. Toor. Comparison of flooding techniques for safety applications in VANETs. In Telecommunications, 200\%. ITST '0\%. '7th International Conference on ITS, pages 1-6, June 2007.

[101] Richard E Neapolitan et al. Learning bayesian networks, volume 38. Prentice Hall Upper Saddle River, 2004.

[102] B Clifford Neuman. Scale in distributed systems. In Readings in distributed computing systems, IEEE Computer Society Press, pages 463-89, 1994.

[103] Hamid Noroozi, Mohammad Khodaei, and Panos Papadimitratos. VPKIaaS: A highly-available and dynamically-scalable vehicular public-key infrastructure. In Proceedings of the 11th ACM Conference on Security 85 Privacy in Wireless and Mobile Networks, WiSec 18, pages 302-304, New York, NY, USA, 2018. ACM.

[104] S. Panichpapiboon and W. Pattara-Atikom. A review of information dissemination protocols for vehicular ad hoc networks. Communications Surveys Tutorials, IEEE, 14(3):784-798, March 2012.

[105] P. Papadimitratos, L. Buttyan, T. Holczer, E. Schoch, J. Freudiger, M. Raya, Z. Ma, F. Kargl, A. Kung, and J. P. Hubaux. Secure vehicular communication systems: design and architecture. IEEE Communications Magazine, 46(11):100109, November 2008.

[106] B. Peng, C. Hu, T. Peng, Y. Yang, and W. Wang. A resource allocation scheme for D2D multicast with QoS protection in OFDMA-based systems. In 2013 IEEE 24th Annual International Symposium on Personal, Indoor, and Mobile Radio Communications (PIMRC), pages 12383-2387, Sept 2013.

[107] T. Peng, Q. Lu, H. Wang, S. Xu, and W. Wang. Interference avoidance mechanisms in the hybrid cellular and device-to-device systems. In 2009 IEEE 20th International Symposium on Personal, Indoor and Mobile Radio Communications, pages 617-621, Sept 2009.

[108] A Qayyum, L. Viennot, and A Laouiti. Multipoint relaying for flooding broadcast messages in mobile wireless networks. In Proceedings of the 35th Annual Hawaii International Conference on System Sciences (HICSS).

[109] H. Rahbar, K. Naik, and A. Nayak. Dtsg: Dynamic time-stable geocast routing in vehicular ad hoc networks. In Ad Hoc Networking Workshop (Med-Hoc-Net), The 9th IFIP Mediterranean, pages 1-7, June 2010.

[110] R. Rajendran and J. de Jongh. An efficient and reliable multi-hop geographical broadcast protocol in vehicular ad-hoc networks. In ITS Telecommunications (ITST), 2013.

[111] G.F. Riley and T.R. Henderson. The ns-3 network simulator. In Klaus Wehrle, Mesut Güneş, and James Gross, editors, Modeling and Tools for Network Simulation. Springer Berlin Heidelberg, 2010. 
[112] F.J. Ros, P.M. Ruiz, and I. Stojmenovic. Acknowledgment-based broadcast protocol for reliable and efficient data dissemination in vehicular ad hoc networks. IEEE Transactions on Mobile Computing, 2012.

[113] William Ryan and Shu Lin. Channel codes: classical and modern. Cambridge University Press, 2009.

[114] R.S. Schwartz, R.R.R. Barbosa, N. Meratnia, G. Heijenk, and H. Scholten. A simple and robust dissemination protocol for VANETs. In Wireless Conference (EW), 2010 European, pages 214-222, April 2010.

[115] R. Scopigno, H.A Cozzetti, A Brofferio, C. Casetti, and C.F. Chiasserini. Mqmapcast: A map-aware protocol for optimized forwarding in urban VANETs. In Connected Vehicles and Expo (ICCVE), 2012 International Conference on, pages 91-98, Dec 2012.

[116] S. Shalmashi, G. Miao, Z. Han, and S. B. Slimane. Interference constrained device-to-device communications. In 2014 IEEE International Conference on Communications (ICC), pages 5245-5250, June 2014.

[117] Baraa T. Sharef, Raed A. Alsaqour, and Mahamod Ismail. Vehicular communication ad hoc routing protocols: A survey. Journal of Network and Computer Applications, 40:363 - 396, 2014.

[118] M. J. Shih, G. Y. Lin, and H. Y. Wei. A distributed multi-channel feedbackless MAC protocol for D2D broadcast communications. IEEE Wireless Communications Letters, 4(1):102-105, Feb 2015.

[119] Ronell B. Sicat. Bit error probability computations for m-ary quadrature amplitude modulation. Digital communications and coding, King Abdullah University of Science and Technology, Thuwal Saudi Arabia.[245] http://in. mathworks. $\mathrm{com} /$ products/matlab, 2009.

[120] M. Slot and V. Cahill. End-to-end acknowledgement of geocast in vehicular networks. In IEEE Vehicular Networking Conference (VNC), 2011.

[121] Laron Smith, Richard Beckman, Doug Anson, Kai Nagel, and Michael Williams. TRANSIMS: Transportation analysis and simulation system. Technical report, Los Alamos National Lab., NM (United States), 1995.

[122] Mark Smith, Gordon Duncan, and Stephen Druitt. PARAMICS: microscopic traffic simulation for congestion management. 1995.

[123] C. Sommer and F. Dressler. Vehicular Networking. Cambridge University Press, 2014. 
[124] Heecheol Song and Hwang Soo Lee. A survey on how to solve a decentralized congestion control problem for periodic beacon broadcast in vehicular safety communications. In Advanced Communication Technology (ICACT), 2013 15th International Conference on Advanced Communications Technology (ICACT), pages 649-654, 2013.

[125] Heecheol Song and Hwang Soo Lee. A survey on vehicle density estimation in vehicular safety communications and its challenging issues. In Intelligent Transportation Systems-(ITSC), 2013 16th International IEEE Conference on Intelligent Transportation Systems, pages 2427-2432, 2013.

[126] I Stojmenovic. Comments and corrections to "dominating sets and neighbor elimination-based broadcasting algorithms in wireless networks". IEEE Transactions on Parallel and Distributed Systems, 15, 2004.

[127] I. Stojmenovic, M. Seddigh, and J. Zunic. Dominating sets and neighbor elimination-based broadcasting algorithms in wireless networks. IEEE Transactions on Parallel and Distributed Systems, 13(1):14-25, Jan 2002.

[128] Sundar Subramanian, Marc Werner, Shihuan Liu, Jubin Jose, Radu Lupoaie, and Xinzhou Wu. Congestion control for vehicular safety: synchronous and asynchronous MAC algorithms. In Proceedings of the ninth ACM international workshop on Vehicular inter-networking, systems, and applications, pages 63-72. ACM, 2012.

[129] W. Sun, D. Yuan, E. G. Ström, and F. Brännström. Resource sharing and power allocation for D2D-based safety-critical V2X communications. In 2015 IEEE International Conference on Communication Workshop (ICCW), pages 2399-2405, June 2015.

[130] M. N. Tehrani, M. Uysal, and H. Yanikomeroglu. Device-to-device communication in $5 \mathrm{G}$ cellular networks: challenges, solutions, and future directions. IEEE Communications Magazine, 52(5):86-92, May 2014.

[131] O.K. Tonguz, N. Wisitpongphan, and Fan Bai. DV-CAST: A distributed vehicular broadcast protocol for vehicular ad hoc networks. Wireless Communications, IEEE, 17(2):47-57, April 2010.

[132] Yu-Chee Tseng, Sze-Yao Ni, Yuh-Shyan Chen, and Jang-Ping Sheu. The broadcast storm problem in a mobile ad hoc network. Wireless Networks, 8(2-3):153$167,2002$.

[133] S. Tsugawa. Inter-vehicle communications and their applications to intelligent vehicles: an overview. In Intelligent Vehicle Symposium, 2002. IEEE, volume 2, pages 564-569 vol.2, June 2002.

[134] Sadayuki TSUGAWA. Issues and recent trends in vehicle safety communication systems. IATSS Research, 29(1):7 - 15, 2005. 
[135] U.F.C. Commision,. "R \& O FCC 03-324," Dedicated Short Range Communications report and order. Technical report, 2003.

[136] S. ur Rehman, M.A. Khan, and T.A. Zia. A multi-hop cross layer decision based routing for VANETs. Wireless Networks, pages 1-14, 2014.

[137] P. Van Mieghem, J. Omic, and R. Kooij. Virus spread in networks. IEEE/ACM Transactions on Networking (TON), 17(1):1-14, Feb 2009.

[138] DSATURN Van Vliet. Saturn-a modern assignment model. Traffic Engineering \& Control, 23(HS-034 256), 1982.

[139] Femke van Wageningen-Kessels, Hans Van Lint, Kees Vuik, and Serge Hoogendoorn. Genealogy of traffic flow models. EURO Journal on Transportation and Logistics, 4(4):445-473, 2015.

[140] W. Viriyasitavat, Fan Bai, and O.K. Tonguz. Uv-cast: An urban vehicular broadcast protocol. In IEEE Vehicular Networking Conference (VNC), 2010.

[141] Vladimir Vukadinovic, Krzysztof Bakowski Patrick Marsch, Ian Dexter Garcia, Hua Xu, Michal Sybis, PawelSroka, Krzysztof Wesolowski, David Lister, and Ilaria Thibault. 3GPP C-V2X and IEEE 802.11p for vehicle-to-vehicle communications in highway platooning scenarios. 74, 032018.

[142] D. Wang, X. Wang, and Y. Zhao. An interference coordination scheme for deviceto-device multicast in cellular networks. In 2012 IEEE Vehicular Technology Conference (VTC Fall), pages 1-5, Sept 2012.

[143] M. Wang, M. Winbjork, Z. Zhang, R. Blasco, H. Do, S. Sorrentino, M. Belleschi, and Y. Zang. Comparison of lte and DSRC-based connectivity for intelligent transportation systems. In 2017 IEEE 85th Vehicular Technology Conference (VTC Spring), pages 1-5, June 2017.

[144] Zhe Wang and M. Hassan. Network coded repetition: A method to recover lost packets in vehicular communications. In IEEE International Conference on Communications (ICC), 2011.

[145] Wikipedia. Absorbing Markov chain — Wikipedia, the free encyclopedia, 2015. [Online; accessed 20-April-2015].

[146] Wikipedia. Hidden Markov model - Wikipedia, the free encyclopedia, 2015. [Online; accessed 20-April-2015].

[147] Wikipedia. Volunteer's dilemma - Wikipedia, the free encyclopedia, 2015. [Online; accessed 10-April-2015].

[148] N. Wisitpongphan, Fan Bai, P. Mudalige, V. Sadekar, and O. Tonguz. Routing in sparse vehicular ad hoc wireless networks. Selected Areas in Communications, IEEE Journal on, 25(8):1538-1556, Oct 2007. 
[149] N. Wisitpongphan, O.K. Tonguz, J.S. Parikh, P. Mudalige, F. Bai, and V. Sadekar. Broadcast storm mitigation techniques in vehicular ad hoc networks. Wireless Communications, IEEE, 14(6):84-94, December 2007.

[150] W.K. Wolterink, G. Heijenk, and G. Karagiannis. Constrained geocast to support cooperative adaptive cruise control (CACC) merging. In Vehicular Networking Conference (VNC), 2010 IEEE, pages 41-48, Dec 2010.

[151] W.K. Wolterink, G. Heijenk, and J.L. van den Berg. An analytical model for the performance of geographical multi-hop broadcast. In Vehicular Networking Conference (VNC), 2012 IEEE, pages 242-249, Nov 2012.

[152] Celimuge $\mathrm{Wu}, \mathrm{S}$. Ohzahata, and T. Kato. How to broadcast efficiently in vehicular ad hoc networks without gps. In Vehicular Networking Conference (VNC), 2012 IEEE, pages 258-264, Nov 2012.

[153] Z. Wu, V. D. Park, and J. Li. Enabling device to device broadcast for LTE cellular networks. IEEE Journal on Selected Areas in Communications, 34(1):5870, Jan 2016.

[154] L. Yang, J. Guo, and Y. Wu. Piggyback cooperative repetition for reliable broadcasting of safety messages in VANETs. In 6th IEEE Consumer Communications and Networking Conference (CCNC) 2009, pages 1-5, Jan.

[155] Dongweon Yoon, Kyongkuk Cho, and Jinsock Lee. Bit error probability of mary quadrature amplitude modulation. In Vehicular Technology Conference Fall 2000. IEEE VTS Fall VTC2000. 52nd Vehicular Technology Conference (Cat. No.00CH37152), volume 5, pages 2422-2427 vol.5, 2000.

[156] Mir Hameed Zeeshan and Fethi Filali. LTE and IEEE 802.11p for vehicular networking: a performance evaluation. EURASIP Journal on Wireless Communications and Networking, 2014(1):89, 2014.

[157] Mir Hameed Zeeshan and Fethi Filali. On the performance comparison between IEEE $802.11 p$ and LTE-based vehicular networks. In Vehicular Technology Conference (VTC Spring), 2014 IEEE 79th, pages 1-5. IEEE, 2014.

[158] Linjuan Zhang, Deyun Gao, Shuai Gao, and V.C.M. Leung. Smartgeocast: Dynamic abnormal traffic information dissemination to multiple regions in VANET. In Wireless Communications and Mobile Computing Conference (IWCMC), 2013 .

[159] Xianfei Zhen, Jie Wang, Ping Wang, Xinhong Wang, and Fuqiang Liu. A senderinitiated adaptive and reliable broadcast scheme for VANET safety message. In International Symposium on Information Science and Engineering (ISISE), 2012. 

3GPP 3rd Generation Partnership Project.

ABS Anti-lock Braking System.

AMC Absorbing Markov Chain.

ARIB Association of Radio Industries and Businesses.

ARQ Automatic Repeat Query.

BER Bit Error Ratio.

BG Broadcast Group.

BLER Block Error Ratio.

BSS Basic Service Set.

C-ITS Cooperative Intelligent Transport Systems.

CA Certification Authority.

CALM Communication Access for Land Mobiles.

CAM Cooperative Awareness Message.

$\mathrm{CCH}$ Control CHannel.

CDF Cumulative Distribution Function.

CMDR Complete Message Delivery Ratio.

CRB Contiguous Resource Blocks.

CRN Cognitive Radio Networking.

CSI Channel State Information.

CSMA Carrier Sense Multiple Access.

CSMA/CA Carrier Sense Multiple Access / Collision Avoidance.

$\mathrm{CU}$ Cellular User.

D2D Device-to-Device.

DAG Directed Acyclic Graph.

DCC Decentralized Congestion Control.

DCI Downlink Control Information.

DECT Digital Enhanced Cordless Telecommunications.

DENM Decentralized Environmental Notification Message.

DL Downlink.

DSRC Direct Short Range Communication.

E2E End-to-End. 
EDCA Enhanced Distributed Channel Access.

eMBB extreme Mobile BroadBand.

eNodeB evolved NodeB.

EPC Evolved Packet Core.

ETSI European Telecommunications Standards Institute.

FDD Frequency Division Duplex.

FEC Forward Error Correction.

FPT First Passage Time.

GDP Gross domestic product.

GPS Global Positioning System.

GSM Global System for Mobile Communications.

HARQ Hybrid Automatic Repeat Query.

IEEE Institute of Electrical and Electronics Engineers.

IID Independent and Identically Distributed.

IoT Internet of Things.

IS Interfering Signal.

ISO international organization for standardization's.

ITS Intelligent Transport Systems.

LTE Long Term Evolution.

MAC Medium Access Control.

MDR Message Delivery Ratio.

MEC Mobile Edge Computing.

MER Message Error Ratio.

MIS Maximum Independent Subset.

mMTC massive Machine-Type Communication.

MS Message Size.

NS-2 Network Simulator 2.

NS-3 Network Simulator 3.

OBU On Board Unit.

OFDM Orthogonal Frequency Division Multiplexing.

OFDMA Orthogonal Frequency Division Multiple Access.

OSI Open System Interconnection.

PDU Protocol Data Unit.

PKI Public Key Infrastructure.

PLR Packet Loss Ratio.

PMF Probability Mass Function. 
PUSCH Physical Uplink Shared CHannel.

QoS Quality of Service.

RB Resource Block.

RE Resource Element.

RFID Radio Frequency IDentification.

RIV Resource Indication Value.

RSU Road Side Unit.

RTS/CTS Request-To-Send / Clear-To-Send.

SC-FDMA Single Carrier-Frequency Division Multiple Access.

$\mathrm{SCH} \quad$ Service CHannels.

SE

Spectrum Efficiency.

SER Segment Error Ratio.

SHB Single-Hop Broadcast.

SINR Signal to Interference plus Noise Ratio.

SIR Signal to Interference Ratio.

SISO Single Input Single Output.

SNR Signal to Noise Ratio.

TB Transport Block.

TBS Transport Block Size.

TDD Time Division Duplex.

TSB Topologically-Scoped Broadcast.

TTI Transmission Time Interval.

UL Uplink.

UMTS Universal Mobile Telecommunication system.

URLLC Ultra Reliable Low Latency Communication.

V2I Vehicle-to-Infrastructure.

$\mathrm{V} 2 \mathrm{~V} \quad$ Vehicle-to-Vehicle.

WAVE Wireless Access for Vehicular Environment.

WHO World Health Organization.

WiFi Wireless Fidelity.

WiMAX Worldwide Interoperability for Microwave Access.

WLAN Wireless Local Area Network. 


\section{List of Publications}

The papers below have been published over the course of this Ph.D. research, sorted in reverse chronological order.

1. M. Gholibeigi, N. Sarrionandia, M. Karimzadeh, M. Baratchi, H. van den Berg, G. Heijenk, Reliable Vehicular Broadcast using 5 G Device-to-Device Communication, IFIP/IEEE WMNC 2017.

2. M. Gholibeigi, M. Baratchi, H. van den Berg, and G. Heijenk, Towards reliable multi-hop broadcast in VANETs: An analytical approach, IEEE VNC 2016.

3. M. Gholibeigi and G. Heijenk, Analysis of multi-hop broadcast in vehicular ad hoc networks: A reliability perspective IFIP/IEEE WD 2016, Best Paper Award.

4. D. Moscoviter, M. Gholibeigi, B.J. Meijerink, R. Kooijman, P. Krijger, and G. Heijenk, Improving Spatial Indexing and Searching for Location-Based DNS Queries, IFIP WG 6.2 WWIC 2016.

5. M. Gholibeigi, G. Heijenk, D. Moltchanov, and Y. Koucheryavy, Analysis of a receiver-based reliable broadcast approach for vehicular networks, Ad Hoc Networks, Special Issue on Advances in Vehicular Networks 2016.

6. M. Gholibeigi, G. Heijenk, D. Moltchanov, and Y. Koucheryavy, Analysis of a receiver-based reliable broadcast approach for vehicular networks, IEEE VNC 2014. 
Mozhdeh Gholibeigi received her B.Sc. degree in Electronics and Electrical Engineering in 2004 from the Urmia University, Iran, and her M.Sc. degree in Communication Networks and Protocols Engineering in 2011 from the Tampere University of Technology, Finland. Prior to beginning her Ph.D., she worked as a researcher at the Polytechnic University of Milan, since 2011 to 2013. Between March 2013 and August 2017, she was a Ph.D. candidate at the Design and Analysis of Communication Systems (DACS) research group of the University of Twente, the Netherlands, under the supervision of Prof. Geert Heijenk and Prof. Hans van den Berg. During this period she took part in the EU FP7 Mobility 2.0 project.

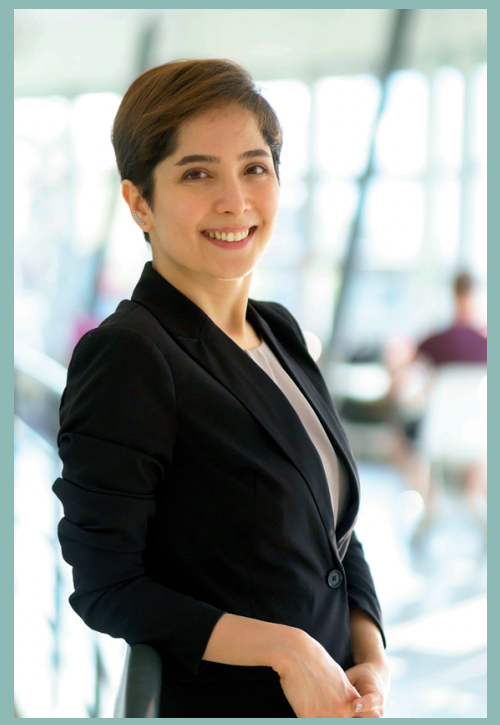

ISSN 2589-7721

ISBN 978-90-365-4690-4

DOI 10.3990/1.9789036546904

https://doi.org/10.3990/1.9789036546904

Copyright @ 2018 Mozhdeh Gholibeigi

(c)

This work is licensed under a Creative Commons

Attribution-NonCommercial-ShareAlike 3.0 Unported License.

http://creativecommons.org/licenses/by-nc-sa/3.0/ 